Linköping University Medical Dissertations No. 1380

\title{
Fast and Accurate 4D Flow MRI for Cardiovascular Blood Flow Assessment
}

Sven Petersson

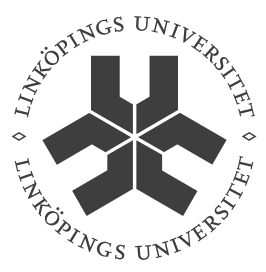

\section{Linköping University \\ FACULTY OF HEALTH SCIENCES}

\author{
Division of Cardiovascular Medicine \\ Department of Medical and Health Sciences \\ Faculty of Health Sciences \\ Center for Medical Image Science and Visualization \\ Linköping University, Sweden
}

Linköping November 2013 
This work has been conducted within the Center for Medical Image Science and Visualization $(C M I V)$ at Linköping University, Sweden. CMIV is acknowledged for provision of financial support and access to leading edge research infrastructure. Furthermore, the Swedish HeartLung foundation and the Swedish Research Council are acknowledged for financial support.

Fast and Accurate 4D Flow MRI for Cardiovascular Blood Flow Assessment

Linköping University Medical Dissertations No. 1380

Copyright (C) 2013 by Sven Petersson unless otherwise stated.

No part of this work may be reproduced, stored in a retrieval system, or be transmitted, in any form or by any means, electronic, mechanic, photocopying, recording or otherwise, without prior written permission from the author.

Division of Cardiovascular Medicine

Department of Medical and Health Sciences

Linköping University

SE-581 85 Linköping Sweden

http://www.liu.se/cmr

ISBN 978-91-7519-506-3

ISSN 0345-0082

Printed by LiU-Tryck Linköping 2013

Cover: Pathline visualization of flow through the left ventricle in a patient. The flow data were acquired using a novel spiral 4D flow MRI sequence. 


\section{Abstract}

The study of blood flow is essential in understanding the physiology and pathophysiology of the cardiovascular system. Small disturbances of the blood flow may over time evolve and contribute to cardiovascular pathology. While the blood flow in a healthy human appears to be predominately laminar, turbulent or transitional blood flow is thought to be involved in the pathogenesis of several cardiovascular diseases. Wall shear stress is the frictional force of blood on the vessel wall and has been linked to the pathogenesis of atherosclerosis and aneurysms. Despite the importance of hemodynamic factors, cardiovascular diagnostics largely relies on the indirect estimation of function based on morphological data.

Time-resolved three-dimensional (3D) phase-contrast magnetic resonance imaging (MRI), often referred to as 4D flow MRI, is a versatile and non-invasive tool for cardiovascular blood flow assessment. The use of 4D flow MRI permits estimation of flow volumes, pressure losses, wall shear stress, turbulence intensity and many other unique hemodynamic parameters. However, 4D flow MRI suffers from long scan times, sometimes over 40 minutes. Furthermore, the accuracy of the many different 4D flow MRI-based applications and estimates have not been thoroughly examined.

In this thesis, the accuracy of 4D flow MRI-based turbulence intensity mapping and wall shear stress estimation was investigated by using numerical simulations of MRI flow measurements. While the results from the turbulence intensity mapping agreed well with reference values from computational fluid dynamics data, the accuracy of the MRI-based wall shear stress estimates was found to be very sensitive to different parameters, especially to spatial resolution, and wall shear stress values over $5 \mathrm{~N} / \mathrm{m}^{2}$ were not well resolved.

To reduce the scan time, a 4D flow MRI sequence using spiral k-space trajectories was implemented and validated in-vivo and in-vitro. The scan time of 4D flow MRI was reduced by more than two-fold compared to a conventional Cartesian acquisition already accelerated using SENSE factor 2, and the data quality was maintained. For a 4D flow scan of the human heart, the use of spiral k-space trajectories resulted in a scan time of around $13 \mathrm{~min}$, compared to $30 \mathrm{~min}$ for the Cartesian acquisition. By combining parallel imaging and spiral trajectories, the total scan time of a 4D flow measurement of the entire heart may be further reduced. This scan time reduction may also be traded for higher spatial resolution.

Numerical simulation of 4D flow MRI may act as an important tool for future optimization and validation of the spiral 4D flow sequence. The scan-time reductions offered by the spiral $\mathrm{k}$-space trajectories can help to cut costs, save time, reduce discomfort for the patient as well as to decrease the risk for motion artifacts. These benefits may facilitate an expanded clinical and investigative use of 4D flow MRI, including larger patient research studies. 



\section{Populärvetenskaplig sammanfattning}

Hjärt- och kärlsjukdom är fortfarande den vanligaste dödsorsaken i Sverige. För att förstå hur det kardiovaskulära systemet fungerar och hur olika sjukdomar uppstår är det viktigt att studera blodflödet samt olika parametrar relaterade till flödet. Turbulent eller stört blodflöde ger upphov till så kallade blåsljud, som är en klassisk indikation på hjärtsjukdom, detekterbar med stetoskop. Turbulent flöde kännetecknas av snabba hastighetsfluktuationer och kan uppstå efter förträngningar av blodkärlen, till exempel efter en aortastenos. Turbulensen leder till en energiförlust, vilket i sin tur leder till att hjärtat får arbeta hårdare. Friktionskraften ifrån blodet på kärlväggen kallas för väggskjuvspänning och har visat sig kunna påverka kärlväggens uppbyggnad samt vara inblandat i uppkomsten av ateroskleros (åderförfettning) och aneurysm. Trots att blodflödet har visat sig ha en stor betydelse för förståelsen av många olika kardiovaskulära sjukdomar har diagnostiken huvudsakligen baserats på funktion uppskattad utifrån morfologi, det vill säga form istället för flöde.

Med en magnetresonanskamera (MR-kamera) är det möjligt att göra tidsupplösta mätningar av blodets hastighet $i$ en tre-dimensionell (3D) volym med hjälp av så kallad fas-kontrast MR. Namnet till trots innebär detta inte att något kontrastmedel injiceras utan att fasen hos den komplexa MR bilden används för att beräkna hastigheten. Utifrån dessa fyrdimensionella $(3 \mathrm{D}+\mathrm{tid}=4 \mathrm{D})$ flödesmätningar går det att beräkna en mängd olika intressanta och kliniskt relevanta parametrar, till exempel turbulensintensitet, väggskjuvspänning, tryckfall och volymflöden. Tidsåtgången för en $4 \mathrm{D}$-flödesmätning kan för vissa tillämpningar överstiga 40 min och de långa mättiderna resulterar i höga kostnader för kliniken, kan innebära obehag för patienten, samt tar tid ifrån andra potentiella undersökningar. För att minska mättiden implementerade vi en mer effektiv mätmetod baserad på spiralformad utläsning.

Det finns också vissa frågetecken angående noggrannheten hos alla de olika mätvärden och parametrar som kan extraheras ur en 4D-flödesmätning. I detta arbete användes datorsimuleringar för att utvärdera noggrannheten av estimering av turbulensintensitet och väggskjuvspänning ifrån 4D flödesdata. Turbulensintensiteten ifrån 4D flödesdata stämde väl överens med referensvärden, medan sambandet mellan estimaten av väggskjuvspänning och faktisk väggskjuvspänning var svagt, speciellt för höga värden.

Genom att använda en spiralformad utläsning av MR-signalen, lyckades vi mer än halvera mättiden. Tidsåtgången för en 4D flödesmätning av hjärtat reducerades ifrån ca 30 min till 13 min med bibehållen god datakvalité. Denna tidsvinst kan främja en utökad tillämpning av 4D flödesmätningar i den kliniska verksamheten, minskar kostnaderna, samt underlättar för större forskningsstudier. Snabba 4D flödesmätningar med hög noggrannhet kan bland annat användas för att öka förståelsen för sambandet mellan blodflödet och hjärt- och kärlsjukdom, underlätta tidigare upptäckt av sjukdom, samt för att planera och utvärdera kirurgiska ingrepp. 



\section{Acknowledgements}

During my work on this thesis, I have had the privilege to be surrounded by many skillful and inspiring persons. First of all I would like to express my sincere gratitude to my supervisor Tino Ebbers who has encouraged and guided me through this work.

Special thanks to my co-supervisors, Petter Dyverfeldt who has contributed a lot with his knowledge and always has been willing to discuss different matters regarding my work, Carl-Johan Carlhäll and Jan Engvall who have shared their expertise and provided me with a physiological perspective on my work.

Further, I would like to thank all present and past members of the cardiovascular MR group at Linköping University that I have had the opportunity to work with. Andreas Sigfridsson and Henrik Haraldsson are acknowledged for many good ideas, discussions and critical contributions to my work. I would like to thank Johan Kihlberg for sharing his knowledge of operating the MRI scanner. Jonatan Eriksson and I have been working parallel on our $\mathrm{PhD}$ studies since the start, thank you for all good discussions and input. I would also like to thank Ann Bolger for constructive comments on my work.

I would like to thank Roland Gårdhagen, Matts Karlsson and Jonas Lantz for contributing with computational fluid dynamics data and knowledge in that field. I am grateful to have had the opportunity to work at the Division of Cardiovascular Medicine and CMIV, and would like to thank all of the staff that have helped me. Marcel Warntjes has contributed with his knowledge of MRI. Elin Wistrand has been of great help for finding solutions to all sorts of administrative matters.

Many thanks to all of my friends for all the good times during these years. Special thanks to my family for all the support and love all these years. Finally, I would like to thank Johanna for your patience, support and love during the final intensive months. I'm looking forward to spending more time with you after this journey has ended.

Sven Petersson

Linköping, November 2013 



\section{List of Papers}

This thesis is based on the following five papers, which will be referred to by their Roman numerals:

\section{PAPER I}

Petersson S, Dyverfeldt P, Gårdhagen R, Karlsson M, Ebbers T

Simulation of Phase Contrast MRI of Turbulent Flow

Magnetic Resonance in Medicine, 2010, 64, 1039-1046.

PAPER II

Petersson S, Dyverfeldt P, Ebbers T

Assessment of the Accuracy of MRI Wall Shear Stress Estimation using Numerical Simulations

Journal of Magnetic Resonance Imaging, 2012, vol 36 issue 1, 128-138.

PAPER III

Sigfridsson A, Petersson S, Carlhäll CJ, Ebbers T

Four-dimensional flow MRI using spiral acquisition

Magnetic Resonance in Medicine, 2012, 68, 1065-1073

PAPER IV

Petersson S, Sigfridsson A, Dyverfeldt P, Carlhäll CJ, Ebbers T

Retrospectively Gated Intra-cardiac 4D Flow MRI using Spiral Trajectories

Submitted.

\section{PAPER V}

Petersson S, Dyverfeldt P, Sigfridsson A, Lantz J, Carlhäll CJ, Ebbers T

Quantification of Stenotic Flow Using Spiral 3D Phase-Contrast MRI

In manuscript.

(Articles reprinted with permission) 


\section{Abbreviations and Nomenclature}

2D

3D

4D Flow

CFD

FVE

IVSD

In vitro

In vivo

$k$-space

$k_{v}$

LDA

LES

LV

MRI

PC

RF

$s(u)$

$S\left(k_{v}\right)$

TE

TKE

TR

$u$

$U$

$u^{\prime}$
Two-dimensional

Three-dimensional

Time-resolved 3D Phase Contrast MRI

Computational fluid dynamics

Fourier velocity encoding

Intravoxel velocity standard deviation

In an artificial environment outside the living organism

Within a living organism

Spatial frequency domain in which the MRI image is being sampled

The applied motion sensitivity of an MRI velocity measurement.

Laser Doppler anemometry

Large eddy simulations

Left ventricle

Magnetic resonance imaging

Phase-contrast

Radio-frequency

The distribution of spin velocities within a voxel

The MRI signal as a function of $k_{v}$ and the Fourier transform of $s(u)$

Echo time

Turbulent kinetic energy

Repitition time

Velocity

Mean velocity

Velocity fluctuation 
VENC Velocity encoding range

Voxel Image volume element, 3D equivalent of a pixel.

WSS Wall shear stress 


\section{Table of Contents}

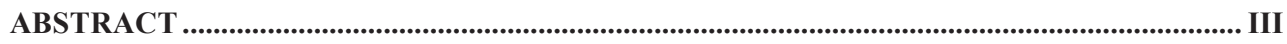

POPULÄRVETENSKAPLIG SAMMANFATTNING ...................................................................... V

ACKNOWLEDGEMENTS.............................................................................................................

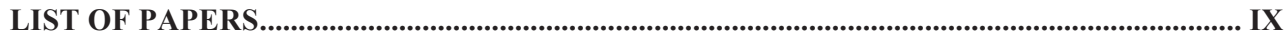

ABBREVIATIONS AND NOMENCLATURE ..................................................................... XI

TABLE OF CONTENTS............................................................................................................. XII

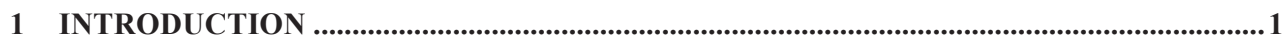

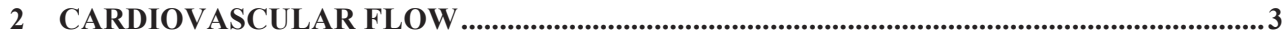

2.1 DISTURBED AND TURBULENT FLOW IN THE CARDIOVASCULAR SYSTEM .................................... 3

3 MAGNETIC RESONANCE IMAGING ...................................................................................5

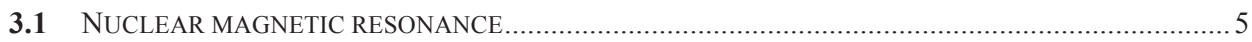

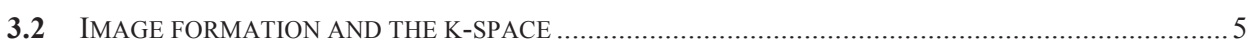

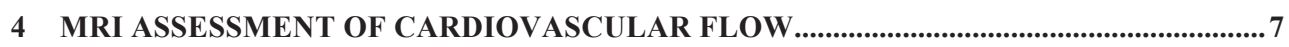

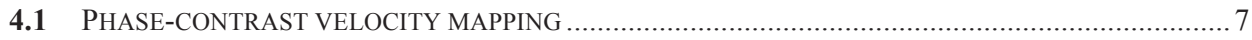

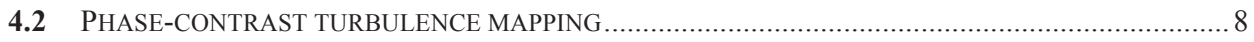

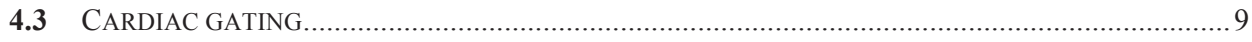

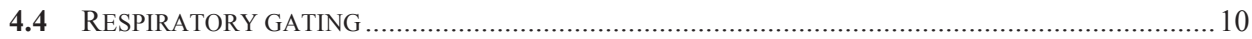

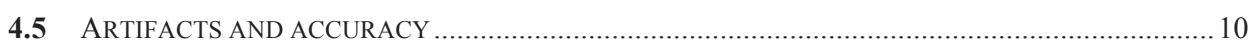

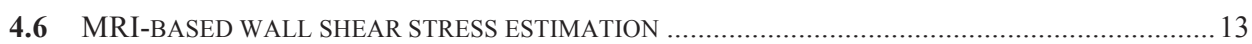

5 REDUCING THE SCAN TIME OF FLOW MRI................................................................

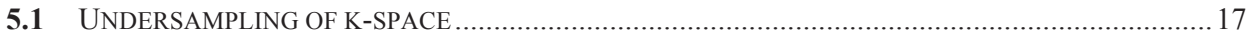

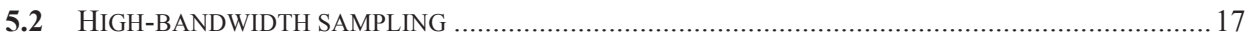

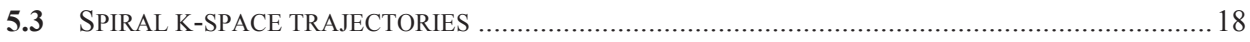

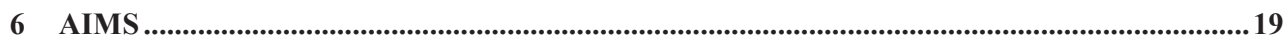

7 NUMERICAL SIMULATION OF PC-MRI MEASUREMENTS .........................................21

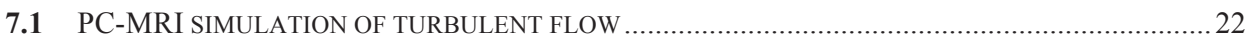

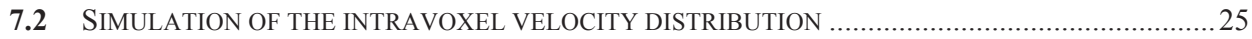

8 ACCURACY OF MRI-BASED WALL SHEAR STRESS ESTIMATION ............................27

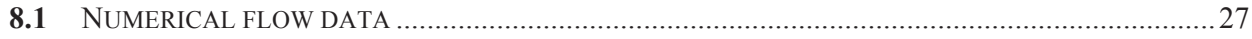

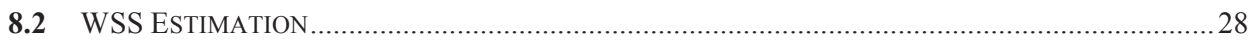

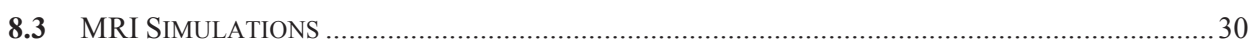

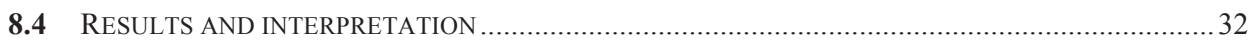




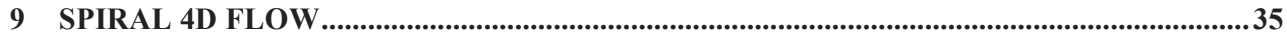

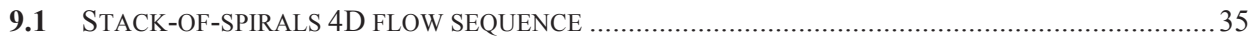

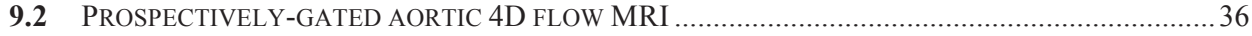

9.3 RETROSPECTIVELY-GATED INTRA-CARDIAC 4D FLOW MRI ....................................................... 41

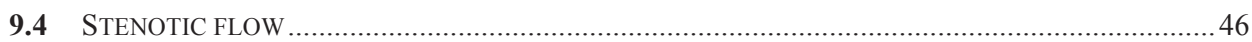

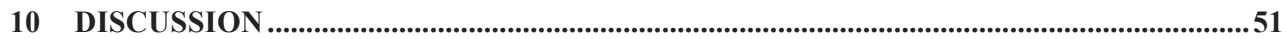

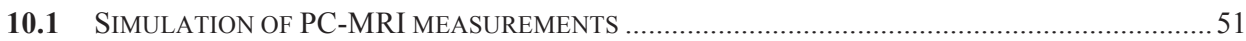

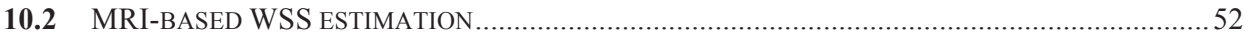

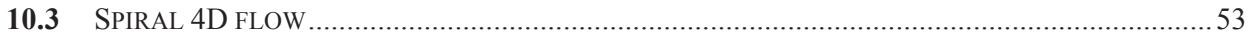

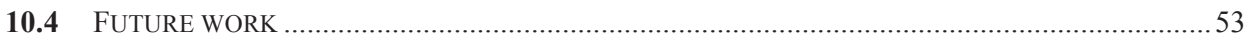

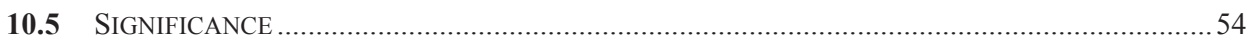

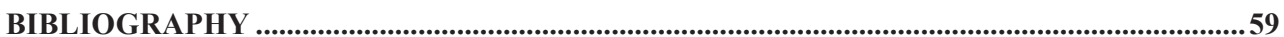




\section{Introduction}

Time-resolved three-directional three-dimensional (3D) phase-contrast (PC) magnetic resonance imaging (MRI), often referred to as 4D flow MRI, is a powerful tool for the noninvasive quantification and visualization of blood flow in the cardiovascular system. The comprehensive nature of 4D flow data permits estimation of volume flow, pressure gradients [1-2], pulse wave velocity [3-5], turbulence intensity [6], wall shear stress (WSS) and many other unique hemodynamic parameters. Such parameters may play an important role in the understanding of the pathophysiology of many cardiovascular diseases which still remain the most prevalent cause of death in the western world [7].

The cardiovascular health care of the aging population includes high-cost pharmaceutical and surgical treatments. Hemodynamic assessment by 4D flow MRI may help to improve outcomes and control costs by evaluation and identification of suitable, cost-effective therapeutic approaches. The use of 4D flow MRI has increased the understanding of normal and abnormal blood flow and has proven valuable in various clinical applications [8-27]. Ample evidence indicates that WSS is involved in the pathogenesis of atherosclerosis and aneurysms [28-33], and an increasing number of studies apply MRI-based WSS estimation invivo [23, 34-43]. However, many cardiovascular applications of 4D flow MRI require large volumetric coverage resulting in scan times of about 20-40 minutes, which may be prohibitive in many cases. Furthermore, the accuracy and feasibility of the many different 4D flow MRIbased applications have not been thoroughly examined. A reduction of scan time in MRI without compromising data quality would be an important step towards expanding the clinical use of 4D flow MRI. 


\section{Cardiovascular flow}

The cardiovascular system is responsible for the transport of oxygen, nutrition and signal substances throughout the entire body. The heart consists of four chambers, the left and right atria and the left and right ventricles. During diastole, the ventricles are filled with blood from the atria, through the mitral and tricuspid valves, respectively. The ventricles then contract and blood is ejected through the aortic and pulmonary valves to the aorta and pulmonary artery, respectively. The blood flow is driven by pressure differences generated by the contraction and relaxation of the heart.

During the development of the cardiovascular system, the heart and vessels will remodel over time in response to flow-induced forces. This remodeling process creates an optimal geometry for efficient flow. However, the same responses to flow-induced forces that shape the developing heart and vessels can also play a role in the pathophysiology of many diseases of the adult cardiovascular system [44]. Small disturbances in blood flow may progress over time and lead to significant adverse remodeling and eventually overt cardiovascular disease.

\subsection{Disturbed and turbulent flow in the cardiovascular system}

Common indicators of cardiovascular disease are the heart murmurs and gallop heart sounds that can be heard using a stethoscope. These heart murmurs are often created by turbulent or disturbed flow, such as the flow through an aortic valve stenosis. Turbulent flow is characterized by fast, and apparently random, velocity fluctuations in space and time. In contrast to laminar flow, which is a streamlined flow where the fluid flows in parallel layers. While the blood flow in a healthy human appears to be predominately laminar, turbulent or transitional blood flow is involved in the pathophysiology of several cardiovascular diseases. For example, flow distortions such as aortic stenosis or prosthetic heart valves can create turbulent flow fluctuations and thereby drastically decrease the transport efficiency of the fluid due to viscous dissipation [45]. This is the major cause of pressure dropping over a constriction, which increases the workload of the heart. Exposure of biological tissue to abnormal turbulent stresses can also cause tissue damage, such as mechanical damage of blood constituents resulting in hemolysis and compromised hemostasis [29].

Atherosclerosis is a chronic inflammatory response to endothelial dysfunction that mainly affects regions of the arterial tree with low or oscillating shear stress [46]. Wall shear stress is the frictional force of the flowing blood on the wall and can affect the endothelial structure [47-48]. Abnormal wall shear stress, hypertension, oxidative stress and elevated low-density lipoproteins are involved in the pathogenesis of atherosclerosis [49-53]. Moreover, studies indicate that wall shear stress is also involved in the pathogenesis of aneurysms $[33,54]$.

Despite the importance of hemodynamic factors, diagnostics of cardiovascular disease relies, to a large extent, on the indirect estimate of function based on morphology. Consequently, suitable tools for hemodynamic assessment are necessary. Cardiac ultrasound is a diagnostic method capable of imaging the anatomy as well as the flow. Doppler velocity ultrasound can measure the flow in a single direction defined by the ultrasound beam and provides a high temporal resolution. In order to obtain the turbulent kinetic energy, turbulence intensity measurements in three directions are necessary, and Doppler ultrasound is only able to 
measure turbulence intensity in one direction. Flow MRI can provide all three velocity components in a $3 \mathrm{D}$ volume and is capable of simultaneous acquisition of velocities and turbulence intensities [6]. 


\section{Magnetic Resonance Imaging}

Magnetic resonance imaging is based on nuclear magnetic resonance property and can be used to visualize internal structures of the human body. The main components of a MRI system are a very strong magnet, typically 1.5-3.0 T, and different coils that are used for receiving and transmitting radio-frequency signals, as well as for spatial encoding.

\subsection{Nuclear magnetic resonance}

Magnetic resonance imaging utilizes the intrinsic magnetism of sub-atomic particles, such as protons and electrons, to obtain both two-dimensional and three-dimensional images of the body. Spin is a fundamental property of such particles, which have a magnetic moment vector in the direction of the spin. The hydrogen nucleus consists of a single proton and is abundant in the human body and the most frequently used particle in MRI. By applying a strong external magnetic field, the spin distribution of the hydrogen nuclei align and start to undergo precession around the external magnetic field. The Larmor frequency, $\omega$, is the frequency of this precession and is proportional to the external magnetic field strength $B_{0}$ and the gyromagnetic ratio, $\gamma$, of the specific particle.

$$
\omega=\gamma B_{0}[\mathrm{rad} / \mathrm{s}]
$$

By applying a radio frequency (RF) pulse rotating with the Larmor frequency in the plane transverse to $B_{0}$, the net magnetization vector of the spin distribution, $\boldsymbol{M}$, is tipped away from the direction of $B_{0}$. After this excitation, the spin distribution will return to its equilibrium, aligned with $B_{0}$. During this relaxation the spins emit a signal that is received using induction in receiver coils. The relaxation of the spins is described by the Bloch equation

$$
\frac{\mathrm{d} \boldsymbol{M}}{\mathrm{d} t}=\gamma \boldsymbol{M} \times \boldsymbol{B}-\frac{M_{x}}{T 2} \hat{x}-\frac{M_{y}}{T 2} \hat{y}-\frac{M_{z}-M_{0}}{T 1} \hat{z}
$$

where $\mathrm{T} 1$ and $\mathrm{T} 2$ are the longitudinal (in the direction of $B_{0}$ ) and transverse relaxation times, respectively. T1 describes the time it takes for the longitudinal component, $M_{\mathrm{z}}$, to reach $63 \%$ of its initial value, $M_{0}$, and T2 describe the time it takes for the transverse components, $M_{\mathrm{x}}$ and $M_{\mathrm{y}}$, to decay to $63 \%$ of their value just after excitation. By using quadrature demodulation, the signal induced in the receiver coils is split up into a real and imaginary part, with a phase difference of $90^{\circ}$. The real and imaginary parts can be seen as a representation of the two transverse components, $M_{\mathrm{x}}$ and $M_{\mathrm{y}}$, respectively. Different tissues have different T1, T2 and density of hydrogen nuclei, and it is these differences that create the contrast in MRI images.

\subsection{Image formation and the k-space}

By applying spatially varying magnetic gradients to $B_{0}$, the spins will precess with different Larmor frequencies depending on their position. The Larmor frequency at position $r$ will be

$$
\omega=\gamma\left(B_{0}+\boldsymbol{G} \cdot r\right)
$$

in the presence of a magnetic field gradient vector $\boldsymbol{G}$. By applying a time varying gradient vector, the spins will accumulate phase according to

$$
\varphi=\gamma \int_{0}^{t} r \boldsymbol{G}(\tau) \mathrm{d} \tau
$$


where $\tau=0$ is the time of excitation. Thus, both phase and frequency can be used to encode the spatial position of the spins. The spatial Fourier domain in which the MRI data are acquired is referred to as the k-space [55-56]. The MRI image is obtained by taking the inverse Fourier transform of the k-space data. The k-space coordinate can be described as

$$
k=\frac{\gamma}{2 \pi} \int_{0}^{t} \boldsymbol{G}(\tau) \mathrm{d} \tau
$$

and the strength of the gradient field, $\boldsymbol{G}$, can be varied over time to traverse the k-space. The k-space can be traversed using an arbitrary trajectory, but the most common approach is to sample the k-space line by line in a Cartesian grid (spin-warp imaging). Moreover, the choice of trajectory is limited by hardware and system imperfections. 


\section{$4 \mathrm{MRI}$ assessment of cardiovascular flow}

\subsection{Phase-contrast velocity mapping}

Phase-contrast magnetic resonance imaging (PC-MRI) is a versatile and non-invasive tool for cardiovascular blood flow assessment and can be applied anywhere in the human body. Conventional PC-MRI velocity mapping measures the mean velocity in each voxel and is used clinically mainly for accurate volume flow measurements. After the development of a PC-MRI approach for the measurement of 3D time-resolved three-directional cardiovascular blood flow [57-59], numerous studies have exploited the technique to describe normal blood flow patterns in, for example, the left atrium [15], left ventricle [16-17], aorta [18], and intracranial vessels [19], as well as the blood flow in different patient groups [20-21, 27, 60$62]$.

PC-MRI velocity mapping exploits the effect of flow on the phase of the MRI signal. By adding a bipolar magnetic field gradient, moving spins will accumulate a phase shift that is proportional to the velocity of the spin (Figure 1). For a stationary spin, the accumulated phase from the first part of the bipolar will be cancelled out by the phase from the second part. Spins moving in the direction of the gradient will experience a difference in gradient field strength during the second part and accumulate a phase shift proportional to the distance the spin has travelled during the bipolar. By dividing this distance by the time, the velocity of the spin is acquired. If the spin velocity distribution in the voxel, $s(u)$, is symmetric around the mean velocity in the voxel, $U$, the phase of the MRI signal in the voxel, $\varphi$, can be described as

$$
\varphi=k_{v} \cdot U+\varphi_{\text {inhom }}
$$

where $k_{v}$ describes the amount of applied first order motion sensitivity and $\varphi_{\text {inhom }}$ is the phase shift caused by factors not related to the motion of the spins, such as the inhomogeneities of the magnetic field.

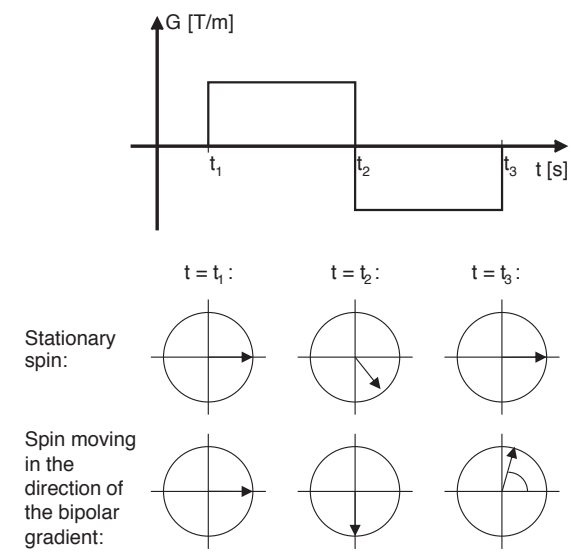

Figure 1. The bipolar magnetic field gradient induces a phase shift proportional to the distance traveled by the spin. Stationary spins will accumulate no motion-induced phase shifts. 
There are different encoding strategies for obtaining the mean velocity of a voxel in all three directions from the phase of the MRI signal [63]. The simple four-point method is probably the most straightforward. A reference scan without any velocity encoding is carried out together with three velocity-encoded scans. By subtracting the phase of the reference scans from the other segments, the unwanted phase term, $\varphi_{\text {inhom }}$, is cancelled out and a phase difference that is proportional to velocity is obtained. The velocity components can then be calculated as

$$
\begin{aligned}
& u_{y}=\frac{\pi}{k_{v}} \cdot \frac{\varphi_{\mathrm{y}}-\varphi_{0}}{\pi} \quad[\mathrm{m} / \mathrm{s}] \\
& u_{y}=\frac{\pi}{k_{v}} \cdot \frac{\varphi_{\mathrm{y}}-\varphi_{0}}{\pi} \\
& u_{z}=\frac{\pi}{k_{v}} \cdot \frac{\varphi_{\mathrm{z}}-\varphi_{0}}{\pi}
\end{aligned}
$$

The amount of motion sensitivity is often described by the velocity encoding range (VENC), which is the velocity corresponding to a phase shift of $\pi$ radians. For the simple four-point method, the VENC is related to $k_{v}$ according to

$$
\mathrm{VENC}=\pi / k_{\mathrm{v}} \quad[\mathrm{m} / \mathrm{s}]
$$

In Section 4.5 some of the different artifacts that may reduce the accuracy of the PC-MRI velocity estimates are described.

\subsection{Phase-contrast turbulence mapping}

Phase-contrast velocity mapping estimates the mean velocities of the voxels, but is not capable of resolving the fast velocity fluctuations present in turbulent flow. However, in phase-contrast turbulence mapping, the intensity of these velocity fluctuations is derived from the magnitude of the PC-MRI signal.

Turbulent flow is characterized by apparently random velocity fluctuations. The velocity fluctuations in an arbitrary direction $i$, can be defined as the difference between the velocity $u_{i}$ and the mean velocity, $U_{i}$.

$$
u_{i}^{\prime}=u_{i}-U_{i}[\mathrm{~m} / \mathrm{s}]
$$

The intensity of the velocity fluctuations can be quantified by their standard deviation, $\sigma_{i}$, which is defined as the root mean square of the velocity fluctuations

$$
\sigma_{i}=\sqrt{\overline{u_{l}^{\prime 2}}}[\mathrm{~m} / \mathrm{s}]
$$

From the intensity of the velocity fluctuations in all three directions, the turbulent kinetic energy (TKE) per unit volume can be obtained as

$$
\mathrm{TKE}=\frac{1}{2} \rho \sum_{i=1}^{3} \sigma_{i}^{2}\left[\mathrm{~J} / \mathrm{m}^{3}\right]
$$

where $\rho$ is the density of the fluid.

The standard deviation of the velocity fluctuations can be estimated from MRI by determining the square root of the second central moment of the intravoxel velocity distribution. 


$$
\sigma=\sqrt{\int_{-\infty}^{\infty}(u-\bar{u})^{2} s(u) d u}[\mathrm{~m} / \mathrm{s}]
$$

This estimate of $\sigma$ is often referred to as the intravoxel velocity standard deviation (IVSD). The IVSD can be estimated from the flow-induced signal loss in PC-MRI data [6, 9, 64]. In the presence of turbulent or disturbed flow, the magnitude of the velocity-encoded MRI signal will decrease due to intravoxel phase dispersion. By modeling the intravoxel velocity distribution as a Gaussian distribution, the IVSD can be computed from the relative signal loss between two velocity-encoded segments.

$$
\operatorname{IVSD}=\frac{1}{k_{v}} \sqrt{2 \ln \left(|S(0)| /\left|S\left(k_{v}\right)\right|\right)} \quad[\mathrm{m} / \mathrm{s}]
$$

where $S(0)$ is the signal from the reference segment and $S\left(k_{v}\right)$ is the signal from the velocityencoded segment with first order motion sensitivity $k_{v}[6]$.

Estimates of IVSD from 4D flow MRI have been shown to agree well with laser Doppler anemometry measurements, particle image velocimetry, and computer fluid dynamics (CFD) simulations of both in-vitro and in-vivo flow [65-67].

\subsection{Cardiac gating}

The beating heart will create a periodically changing pulsatile flow, and if the changes in flow and movement of the heart not are accounted for, the image will be distorted by motion artifacts. By acquiring the patient's electrocardiogram (ECG) while in the MRI scanner, a MRI measurement can be synchronized to the heart cycle. Typically an MRI measurement is much longer than a single heartbeat and covers a large number of heartbeats that are averaged into a time-resolved image of one single heartbeat. The two main approaches to cardiac gating are prospective and retrospective gating [68-69]. In prospective cardiac gating, the sequence triggers on the R pulse of the ECG (indicating the onset of systole) and then sampling is carried out at specific time intervals. One drawback of this approach is that prospective gating does not cover the complete heart cycle, as the end of diastole is not sampled. This problem can be solved by using retrospective cardiac gating, in which sampling occurs continuously and every sample is assigned a trigger time. The trigger time is the time since the latest $\mathrm{R}$ pulse and the samples are retrospectively sorted to create time-resolved images of the complete cardiac cycle. For cardiac 4D flow, the ability to track blood over the complete cardiac cycle is crucial, and allows for pathline-based quality assessments [70].

As the length of the heart cycle varies with varying heart rate, heartbeats have to be normalized to the same length before the retrospective interpolation. Systole typically accounts for only a small fraction of the change in heart rate. A non-linear stretch where diastole is stretched more than systole can be used to compensate for this.

In conventional three-directional PC-MRI velocity mapping, four scan segments with different velocity encodings are obtained. The order in which these scan segments are acquired may be changed. Segmented k-space sampling can be used to acquire several kspace lines, or in this case several scan segments for each heart cycle, at the cost of reduced temporal resolution. By using a repetition time (TR) interleaved approach, all four segments are acquired sequentially during every heartbeat. The nominal temporal resolution for a TRinterleaved sequence will be four TR. If a higher temporal resolution is desired, a beatinterleaved sequence can be used. In the beat-interleaved sequence, only one segment is acquired during one heartbeat and the next segment is acquired during the following heartbeat. This results in a temporal resolution of one TR, however, scan time will be 
increased four-fold. Moreover, the beat-interleaved sequence is more sensitive to beat-to-beat variations compared to the TR-interleaved. The scan time of a beat-interleaved or TRinterleaved sequence can be reduced by sampling several $\mathrm{k}$-space lines per heart cycle, but this will result in reduced temporal resolution.

\subsection{Respiratory gating}

As the heart is attached to the diaphragm, the movement of the diaphragm during respiration will cause the heart to move. Moreover, respiration affects the pressure in the thorax, which in turn affects the flow in the heart. In order to avoid motion artifacts, not only cardiac gating is necessary but also respiratory gating or breath-hold acquisitions. Respiratory gating can be performed by applying a navigator pulse before every heartbeat, estimating the position of the diaphragm. Typically measurements are then only carried out at the end expiration phase, which results in a considerable increase in scan time as only around $50 \%$ of the time is used for measuring. Alternatively, the respiratory cycle can be gated in a similar manner as the cardiac cycle, which may unveil important hemodynamic information but will further increase scan time [71-72].

\subsection{Artifacts and accuracy}

Ideally, the B0 field would be a homogeneous magnetic field and the gradients and resulting $\mathrm{k}$-space trajectories would behave exactly as designed, but the reality is seldom as perfect as described in the equations in Chapter 3 and the accuracy is also limited by the signal to noise ratio (SNR). Moreover, many PC-MRI artifacts are more prominent in the complex and disturbed flows often associated with cardiovascular disease.

\subsubsection{Signal to noise ratio}

The SNR of a MRI measurement is proportional to the field strength but is also affected by many other factors, such as the coils, spatial resolution, temperature and reconstruction. Therefore, it is non-trivial to obtain an objective estimate of the SNR of an MRI image, especially as the reconstruction often includes a fair amount of filtering, resampling, zeropadding and other operations. The velocity to noise ratio in PC-MRI velocity mapping can be calculated as a function of the SNR and the VENC.

$$
\mathrm{VNR}=\frac{\pi}{\sqrt{2}} \frac{U}{\mathrm{VENC}} \mathrm{SNR}
$$

For accurate turbulence estimates, the VENC must be low enough to obtain a significant signal loss but not so low that the remaining signal magnitude is in the order of the magnitude of the noise [73].

\subsubsection{Magnetic field and RF field inhomogeneities}

An inhomogeneous magnetic field will affect spatial encoding and the relaxation of the spins, which will distort the image. Imperfections of the gradients will have a similar effect, and consequently, the Larmor frequency at different positions will deviate from its assumed value.

There are several potential causes of an inhomogeneous magnetic field. Imperfections of the magnet or external ferromagnetic objects influence the magnetic field. Differences in magnetic susceptibility of materials imaged will also result in an inhomogeneous magnetic field. Correction of the inhomogeneity can be achieved by shimming the scanner by adding a correction magnetic field (active shimming) or by placing metal pieces within the bore of the 
magnet to reach a more homogeneous state (passive shimming). However, some inhomogeneity will still be present, especially at higher field strengths where correction is more difficult. Moreover, there may be inhomogeneities in the RF field. Metal in the body will cause severe artifacts due to susceptibility and RF inhomogeneity. For example, RF inhomogeneity results in a signal void around metal aortic stents or metal dental work, which prohibits imaging of these regions.

Chemical shift is the misregistration of, for example, fat and water-based tissues due to differences in resonance frequency. The resonance frequency differs by around $210 \mathrm{~Hz}$ at 1.5 $\mathrm{T}$ and $420 \mathrm{~Hz}$ at 3T. In Cartesian spin-warp imaging, this difference results in a misregistration in the frequency-encoding direction.

\subsubsection{Eddy currents and Maxwell effects}

Eddy currents are electric currents induced in the receiver coils by changes in the magnetic field. The gradients in an MRI sequence may change rapidly and the eddy currents created will, in turn, induce a magnetic field. Shielded gradient coils and design of the gradient sequence can limit eddy currents. Concomitant gradient field effects are additional gradient fields in the other directions, in accordance with the Maxwell equations [74]. Both eddy currents and concomitant gradient fields result in spatially varying phase shift. However, the concomitant gradient fields can be computed and corrected for [74]. Eddy currents are more difficult to correct for and often require some assumption of how the phase shift varies over the image.

It is possible to correct for eddy currents by repeating the measurement on a stationary phantom and subtract the velocities from the stationary phantom from the prior measurement. However, this is very cumbersome as scan time and amount of data are doubled. An approach often applied in-vivo is to fit a spatially varying polynomial expression to the stationary tissue and subtract this expression from the flow field [75] [76]. However, a low order polynomial (1-2) may not be enough to remove the phase offsets and a high order polynomial may induce phase shifts if incorrect. The method is also limited by the amount of stationary tissue surrounding the region of interest.

\subsubsection{Motion and displacement artifacts}

Motion during imaging, for example, respiratory movement, will create motion blurring or ghosting if not accounted for. Moreover, spatial misregistration (displacement) of moving objects may occur, as there is a short delay between the spatial phase and frequency encoding. For example, flow with velocity components in both the phase and frequency-encoding direction will be displaced as showed in Figure 2. The amount of displacement will be proportional to the velocity and the delay between the different encodings. 


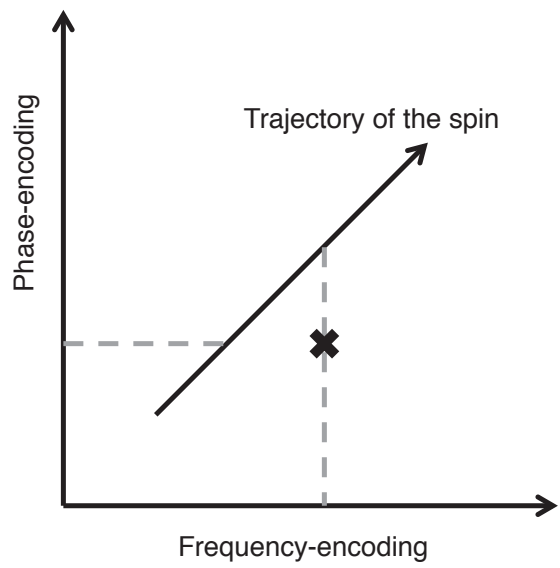

Figure 2. As there is a small delay between phase-encoding and frequency encoding, a spin moving in an oblique direction will be displaced to the position denoted by the $\mathrm{x}$. The dashed lines show the position encoded in the phase and frequency encoding directions, respectively.

Acceleration and higher orders of motion may also induce phase shifts resulting in incorrect velocity estimates. These phase shifts can be interpreted as a displacement of the velocities due to acceleration of the spins between the velocity and spatial encoding [77]. For example, the flow through an aortic valve stenosis will be highly accelerating, resulting in a displacement of the velocity values if the flow is in a non-phase-encoding direction. As phase encoding is usually carried out simultaneously with the velocity encoding, no displacement occurs in the phase-encoding direction. As the time between velocity encoding and spatial encoding is known, it is possible to correct for these artifacts to some extent [78].

Periodic motion, such as breathing or pulsatile flow, may induce ghosting artifacts in the phase-encoding directions. Moreover, turbulent or disturbed flow will cause more random ghosting artifacts in the phase-encoding direction due to variations of the flow between different readouts.

\subsubsection{Velocity Aliasing}

If any of the velocity components exceed the VENC, phase aliasing will occur. For example, a velocity of $1.5 \cdot \mathrm{VENC}$ will result in a phase shift of $270^{\circ}$, but as this is more than $180^{\circ}$ this phase shift will be interpreted as $-90^{\circ}$, which results in a velocity of $-0.5 \cdot \mathrm{VENC}$. The same thing occurs for velocities under -VENC. This velocity aliasing can be avoided by settings a VENC that exceed the expected maximum velocity. However, as stated previously, a higher VENC results in lower VNR. Methods for unwrapping velocity aliasing are available, but do not always succeed in unwrapping all aliasing [79-80].

\subsubsection{Partial Volume Artifact}

Partial volume artifacts occur when a voxel contains different tissues. The value of a voxel containing different tissues will be a complex sum of the signals from the different tissues. For example, at the proximity of the wall, voxels containing both vessel wall and blood lumen may give incorrect velocity estimates depending on the signal magnitude of both these tissues. Flowing blood is supplied with new unsaturated spins, which increase the signal of the blood. 
If the signal of the blood is larger than the signal of the vessel wall, the mean velocity in the voxel may be overestimated by PC-MRI, and the other way around.

\subsubsection{Intravoxel phase dispersion}

Intravoxel phase dispersion acts in a similar fashion to partial volume effects. If a voxel contains spins with different velocities or higher order motions such as acceleration, the phase differences in the voxel will cancel each other out resulting in a decrease in signal magnitude in the voxel. This phase dispersion will result in a signal void in turbulent flow, which can be used to quantify the turbulence as described in Section 4.2. The amount of signal loss can be limited by using a shorter TE [81].

\subsubsection{Fold over}

In accordance with Nyquist theorem, the field of view (FOV) of a MRI image is related to the sampling density in k-space, $\Delta k$, as

$$
\mathrm{FOV}=\frac{1}{\Delta k}
$$

Signal outside the FOV will be aliased back into the image creating fold-over artifacts. In spin-warp imaging fold-over artifacts appear in the phase-encoding direction. For other kspace trajectories, such as spiral or radial, the fold-over artifacts will be more complex as the sampling density is varying.

\subsection{MRI-based wall shear stress estimation}

Wall shear stress is the frictional force from the blood on the vessel wall and can be computed as

$$
\text { WSS }=\mu\left(\frac{\delta u}{\delta x}\right)_{x=0}\left[\mathrm{~N} / \mathrm{m}^{2}\right]
$$

where $\mu$ is the dynamic viscosity, $u$ is the velocity parallel to the wall and $x$ is the distance from the wall in the direction of the wall's inward normal. Although this expression is fairly simple, the computation of WSS from MRI velocity data is a far from easy task. Partial volume artifacts distort velocity estimates in the proximity of the wall. Finding the position of the wall can be difficult, especially as contrast may be poor and the wall is in motion. Small errors in velocity estimates can translate into large errors when estimating the velocity derivative. The estimation of WSS is especially difficult for steep velocity gradients, such as for plug-like flow profiles. Many of these difficulties are related to the limited spatial and temporal resolution of MRI velocity mapping. In order to visualize how rapidly the velocity gradient can increase when approaching the wall and the complexity of the flow in-vivo, Figure 3 shows the velocity in a cross section of the aorta from a CFD simulation of a healthy volunteer based on a time-resolved segmentation (J. Lantz, personal communications, October 4, 2013). 


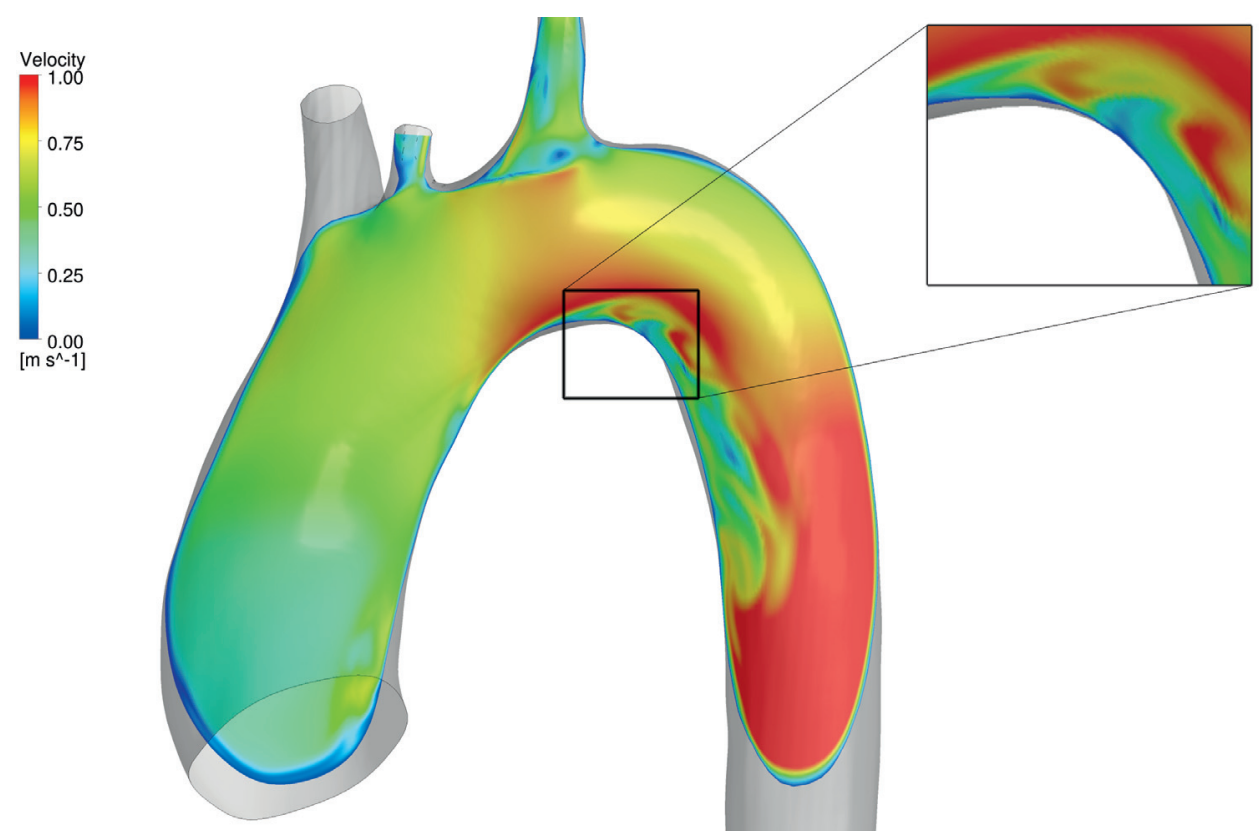

Figure 3. A snapshot of the magnitude of the velocity in a cross section of the aorta during systole from a subject-specific CFD simulation based on a time-resolved segmentation of a healthy volunteer. The velocity is color-coded according to the color bar. The box shows a zoomed-in region of the aorta.

Wall shear stress can also be estimated from Doppler velocity measurements by assuming fully developed Poiseuille or Womersley velocity profiles. However, comparison with computational fluid dynamics data shows major errors, even in vessels considered long and straight [82].

The increasing number of studies applying the MRI-based WSS estimation in-vivo [23, 3443] prompt detailed investigations of the accuracy of MRI-based WSS estimation. Estimates of WSS from 4D flow data in an intracranial aneurysms showed large discrepancies compared to subject-specific CFD simulations [36]. Although some validation has been performed for most MRI-based WSS estimation methods, it is difficult to compare the accuracy of the different methods. Different flow cases and ranges of WSS have been studied. The influence of parameters such as VENC, spatial resolution and segmentation errors has not been extensively investigated. Moreover, subject-specific CFD studies indicate that the magnitude of WSS in a healthy aorta, carotid arteries and cerebral aneurysms may reach up to $30 \mathrm{~N} / \mathrm{m}^{2}$ [83-84], $5 \mathrm{~N} / \mathrm{m}^{2}$ [85] and $20 \mathrm{~N} / \mathrm{m}^{2}$ [54], respectively. In the presence of disturbed flow, such as in the vicinity of a stenosis, much higher WSS values may be present. As many of the methods have been validated for much lower values, sometimes below $1 \mathrm{~N} / \mathrm{m}^{2}$, evaluation of the accuracy for physiological WSS values is lacking. Because of the limitations of MRIbased WSS estimation, it has been suggested that MRI-based WSS estimation is only suitable for obtaining the relative WSS distribution, for example, visualizing and differentiating areas of high and low WSS. However, it remains unclear if the MRI-based WSS estimates are monotonically related to actual WSS values. 


\subsubsection{Linear velocity profile estimation}

There are numerous methods for MRI-based WSS estimation. The most basic methods estimate the velocity gradient from two or more voxels adjacent to the wall using linear extrapolation [86]. By assuming a linear velocity profile close to the wall and using the velocity values from a voxel at the wall, $u_{1}$, and its adjacent voxel in the lumen, $u_{2}$, the spatial velocity derivative can be estimated as

$$
\frac{\delta u}{\delta x}=\frac{u_{2}-u_{1}}{\Delta x}
$$

where $\Delta x$ is the spatial resolution. This approach has been refined to account for partial volume artifacts, utilizing the principle of mass conservation to estimate the subvoxel position of the wall [87]. However, the assumption of a linear velocity profile is expected to result in an underestimation of the spatial velocity derivative at the wall as the velocity gradient typically increases rapidly when approaching the wall.

\subsubsection{Parabolic and cubic spline-based methods}

By fitting a parabolic velocity profile to three adjacent voxels, increased accuracy can theoretically be obtained. The linear extrapolation method has been shown to consistently underestimate WSS for reference WSS values between 1 and $6 \mathrm{~N} / \mathrm{m}^{2}$, and while the parabolic approach did perform better, errors up to $36 \%$ were present (reconstructed pixel size $0.7 \times 0.7$ $\mathrm{mm}^{2}$ ) [88]. The parabolic approach has been extended to applying a 3D parabolic profile to the boundary layer [89]. Womersley velocity profile fitting has also been used to estimate WSS in pulsatile flow [34].

These methods were developed for 2D through-plane velocity MRI and are only valid under the assumption that in-plane velocities in the circumferential direction are negligible and that the normal vector of the wall is in the $2 \mathrm{D}$ plane. Complex flows including helical flow patterns have been shown to be present in large and medium-sized arteries even in healthy volunteers [90-91].

Level set-based segmentation of the wall together with cubic Lagrangian interpolation has been used for MRI-based WSS estimation in large vessels [92]. According to the no-slip condition, the velocity should be zero at the wall. However, in order to limit sensitivity to segmentation errors and movement of the vessel wall, the velocity values were interpolated at the level set segmentation boundary. The level sets account for the moving wall, and although this approach was validated for $2 \mathrm{D}$ through plane velocity data, it should be possible to extend to three-directional 3D measurements. Moreover, this approach requires no assumptions on the shape of the lumen. A similar approach using cubic splines for segmentation and velocity interpolation has been used to estimate both the axial and circumferential WSS components from 4D flow MRI data [38]. This approach has been evaluated by numerical simulations of parabolic flow with a WSS of $0.6 \mathrm{~N} / \mathrm{m}^{2}$ for which the WSS was underestimated by $40 \%$ using a spatial resolution of $1 \mathrm{~mm}$ in a noise free environment [38]. The reproducibility of the cubic spline approach has been evaluated in-vivo and while spatiotemporal relative WSS distributions could be reliably replicated, the robustness of global and regional WSS was found to be limited and the estimates varied depend on spatial resolution [37-38].

Radial imaging is suitable for high-resolution 4D flow MRI, and phase contrast vastly undersampled isotropic projection (PC-VIPR) MRI [93] has been used for WSS estimation by cubic splines in patients with ascending aortic dilatation and arteriovenous malformations [35, 39]. By using radial imaging, a spatial resolution $0.67-1.4 \mathrm{~mm}$ was obtained, while in 
conventional Cartesian imaging the spatial resolution is usually $1.5-3 \mathrm{~mm}$. This increase in spatial resolution should theoretically improve the accuracy of WSS estimates. In a comparison of a VIPR and a stack-of-stars (radial in-plane sampling) sequence, with spatial resolutions $0.67 \times 0.67 \times 0.67 \mathrm{~mm}^{3}$ and $0.4 \times 0.4 \times 1 \mathrm{~mm}^{3}$, respectively, the time-averaged axial WSS was more than $50 \%$ higher for the stack-of-stars sequence [94]. These findings indicate a large sensitivity to spatial resolution.

\subsubsection{Fourier velocity encoding and IVSD-based methods}

Fourier velocity encoding (FVE) is capable of estimating the intravoxel velocity distribution by sampling the $k_{v}$-space, which is the velocity equivalent of $\mathrm{k}$-space. By assuming that the velocity profile in the voxel monotonically decreases when approaching the wall, a sorting algorithm can be used to estimate an intravoxel velocity profile from which the WSS can be estimated at sub-voxel resolution [95]. However, FVE is very time-consuming as one measurement is necessary for every $k_{v}$. Spiral trajectories have been used to decrease the scan time of FVE measurements used for WSS estimation [96].

As previously described, the IVSD can be obtained from the magnitude of a standard PC-MRI measurement by assuming a Gaussian intravoxel velocity distribution [6]. This method has been optimized to estimate the WSS by estimating the mean velocity gradient in the voxel [97]. The estimation of WSS from IVSD will be further examined in Chapter 8 .

By using the FVE or IVSD-based WSS estimation methods, an estimate of the WSS can be obtained from a single voxel, in contrast to the other methods described where two or more voxels are necessary.

\subsubsection{Image-based CFD simulations}

Some of the limitations of image-based WSS estimation can be avoided by using subjectspecific image-based CFD simulations for WSS estimation [33, 36, 84, 98], which can offer a very high spatial resolution close to the wall. This technique is often limited by the assumption of rigid walls. However, recently CFD simulations incorporating fluid-structure interaction have been used to simulate the flow in a subject-specific aorta [99]. It was found that the influence of wall motion was low on time-averaged WSS and oscillating shear index, but a clear difference was present for instantaneous WSS values. 


\section{Reducing the scan time of flow MRI}

Several techniques for reducing the scan time of MRI measurements are available. This chapter provides a brief overview of the most common methods used for reducing the scan time of MRI-based flow assessments. For example, a reduction in scan time can be achieved by data undersampling or by more efficient sampling of k-space.

\subsection{Undersampling of k-space}

Parallel imaging is one of the most commonly used approaches for decreasing the scan time of flow MRI. By utilizing the coil sensitivity variations of multiple coil elements, undersampled data can be reconstructed without aliasing artifacts (fold-over). Two of the most commonly used parallel imaging methods are SENSE (SENSitivity Encoding) and GRAPPA (GeneRalized Autocalibrating Partially Parallel Acquisitions) [100-101]. However, the drawbacks of data undersampling include reduced SNR or temporal smoothing.

Spatiotemporal parallel imaging utilizes correlations in both k-space and time to reduce the number of samples necessary for reconstruction of the image data [102-104]. Methods such as k-t SENSE and k-t GRAPPA have been used to reduce scan times for Cartesian PC MRI, reaching net acceleration factors of around 5 [105-107]. While some temporal smoothing remains for k-t approaches, especially for high reduction factors, principal component analysis has shown promising results for reduction factors of up to 8 for 4D flow (k-t PCA) [108].

Compressed sensing in combination with Poisson disk undersampling has also been used to accelerate 4D flow MRI, reaching net acceleration factors of 2 to 5 [109-110].

PC-VIPR MRI using 3D radial k-space trajectories has been used to reduce the scan times of 4D flow scans of the aorta and heart with high spatial resolution [93, 111-112]. Furthermore, a stack of 2D radial trajectories (stack of stars) accelerated using undersampling and temporal view-sharing has been used for 4D flow imaging in intracranial aneurysms [113]. The use of PC-VIPR enables very high acceleration factors for high-spatial-resolution scans, but SNR and streaking artifacts limit the amount of acceleration.

\subsection{High-bandwidth sampling}

The k-space can be traversed faster by using a higher bandwidth, i.e. stronger gradients for spatial encoding. However, in Cartesian spin-warp imaging a large part of every TR is used for excitation and velocity encoding and a relatively small part is used for reading data. Consequently, the use of an increased bandwidth for spin-warp imaging is not very costeffective as the readout is already relatively short. Using spiral k-space trajectories, a larger part of the TR is usually used for reading data and high-bandwidth sampling can be used to sample a large fraction of k-space in a short period of time. Moreover, a higher bandwidth reduces the chemical shift and susceptibility effects. 


\subsection{Spiral k-space trajectories}

Spiral k-space trajectories are very efficient as a large part of the TR can be spent reading data. The spiral readouts start in the center of k-space resulting in a shorter TE and less T2* signal decay in the center of k-space. The high level of efficiency of spiral trajectories allows a reduction in scan time without a reduction in SNR. The combination of spiral trajectories and high-bandwidth sampling can be used to further decrease scan times. However, the use of spiral k-space trajectories has been limited by sensitivity to system imperfections and off resonance due to magnetic field inhomogeneities and chemical shifts. As hardware and correction methods have been improved, these effects may be limited or corrected for. A schematic illustration of Cartesian, radial and spiral k-space trajectories is shown in Figure 4.
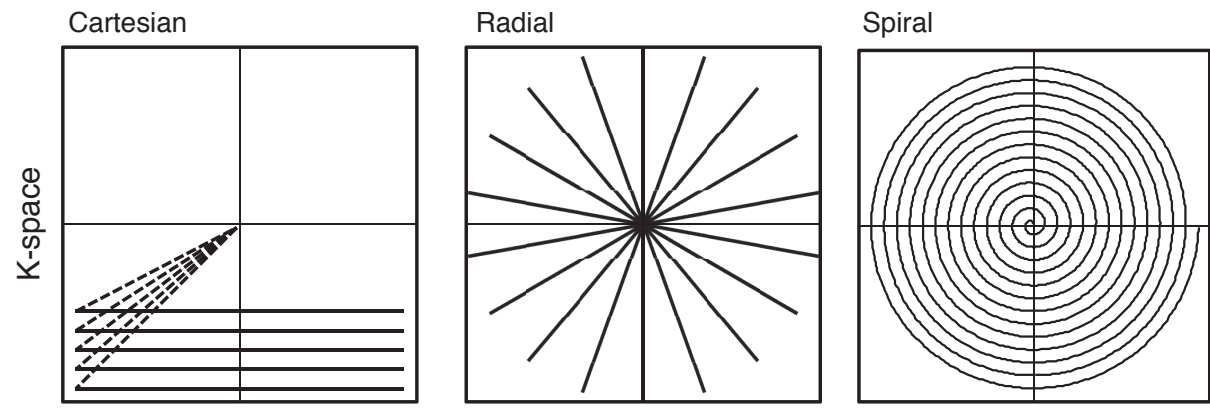

Figure 4. Schematic figures of Cartesian (spin-warp imaging), radial and spiral k-space trajectories.

Spiral k-space trajectories have previously been used for real time and three-directional single-breath-hold 2D flow MRI [114-116] and 3D magnetic resonance angiography [117119]. Recently, spiral trajectories have also been used for prospectively-gated 4D flow MRI in the carotid arteries [120] and the aorta (Paper III). Moreover, in Paper IV a retrospectivelygated 4D flow MRI sequence using spiral trajectories was validated for velocity mapping of intra-cardiac velocities.

Using spiral trajectories, fold over will appear as less structured swirl artifacts in the spiral plane. These artifacts are most prominent close to the edge of the image and may be reduced by using variable density spiral trajectories with denser sampling in the center of k-space [121]. Spiral sampling is more sensitive to magnetic field inhomogeneities than Cartesian sampling. Main field inhomogeneities, gradient imperfections, eddy currents, chemical shifts, susceptibility artifacts and concomitant gradient effects will all contribute to blurring of the image. The amount of blurring will be related to the length of the readout and can therefore be limited by using short interleaved spiral readouts. Several methods for correction of these blurring artifacts exist, but they are often computationally demanding and require an additional $B_{0}$ field map measurement [122]. 


\section{Aims}

The overall aim of this work was to evaluate the accuracy and decrease the scan times of 4D flow MRI without compromising data quality. Specific aims addressed are:

- To develop numerical simulations methods for evaluating the accuracy of 4D flow MRI.

- To evaluate the accuracy of 4D flow MRI-based WSS estimation.

- To decrease the scan time of 4D flow MRI by utilizing spiral k-space trajectories.

- To evaluate the feasibility of spiral 4D flow MRI in the healthy heart, aorta and for stenotic flow, and assess its accuracy in relation to conventional Cartesian 4D flow MRI. 


\section{Numerical simulation of PC-MRI measurements}

Many PC-MRI artifacts are more prominent in the different types of disturbed and complex flow that accompany many cardiovascular diseases. The actual flow in a PC-MRI measurement is unknown, and no apparent gold standard exists for flow measurements invivo. Consequently, it is difficult to determine the accuracy and extent of different artifacts from studying measurements only. Simulations of PC-MRI measurements from numerical flow data permit investigations of the outcome of a measurement on a known flow field. The numerical flow data may be a simple parabolic flow profile, as well as advanced patient specific computational fluid dynamics (CFD) simulations of more complex flows. By using simulations, in-depth studies of artifacts and validation of different PC-MRI sequences and applications can be made. In this chapter, a method for the simulation of PC-MRI of turbulent flow is presented along with a more simple simulation approach based on estimation of the intravoxel velocity distribution.

In previous studies, several different approaches to simulating PC-MRI measurements have been used. An Eulerian-Lagrangian approach has been used to simulate 2D through-plane PCMRI from CFD data of flow with low Reynolds number [123-124]. In an Eulerian specification of the flow field, the flow is described for fixed locations. In contrast to a Lagrangian specification, where the flow is described from the point of view of a virtual particle in the fluid moving through space and time. In the Eulerian-Lagrangian approach, the flow data are first obtained by CFD simulations on a fixed mesh. The Bloch equation is then solved for virtual particles travelling along their trajectories.

While the Eulerian-Lagrangian simulation approach is fairly accurate and straightforward, the application is limited by excessively long computation times, in some cases requiring several days for the simulation of a single measurement. Pathlines for a large number of particles are necessary to estimate the average phase in a voxel. Intravoxel phase dispersion effects may also be inaccurately simulated as the distribution of particles during the readout will depend on the flow field and voxels may contain different amounts of particles, contradicting the principle of mass conservation.

A strict Eulerian approach for solving the Bloch equations has been used for simulation of MRI of pulsatile flow in the carotid bifurcation [125-126]. As this approach does not comprise the computation of particle trajectories, it is much less computationally expensive. Intravoxel phase dispersion is also correctly simulated, as the amount of particles in every voxel is constant and defined by the mesh. However, the Eulerian approach is not valid for unsteady flows if the time scale of velocity fluctuations is of the same order or shorter than the time scale of the MRI sequence [126]. Consequently, the Eulerian approach is less appropriate for the simulation of turbulent or disturbed flow. Moreover, misregistration artifacts are naturally included using the Eulerian-Lagrangian approach, whereas they have to be modeled in the Eulerian approach by a transformation of the mesh [125]. 


\subsection{PC-MRI simulation of turbulent flow}

As turbulence accompanies many cardiovascular diseases and many PC-MRI artifacts are more prominent in turbulent flow, tools for quality control and validation of PC-MRI measurements of turbulent flow are required. In Paper I, an Eulerian-Lagrangian approach was used to simulate 3D PC-MRI measurements of turbulent flow. The method was validated by comparison with in-vitro PC-MRI measurements.

\subsubsection{Numerical flow data}

Numerical flow simulations resolving turbulent velocity fluctuations were obtained using large eddy simulations (LES), which have been validated against laser Doppler anemometry and direct numerical simulations [127-128]. Non-pulsatile flow with a Reynolds number of 1000 at the inlet in a straight rigid pipe with a stenosis with an area reduction of $75 \%$ and a diameter of $14.6 \mathrm{~mm}$ was simulated (Figure 5). The fluid considered had a kinematic viscosity of $0.12 \cdot 10^{-4} \mathrm{~m}^{2} / \mathrm{s}$ to mimic the properties of blood. A fully developed laminar velocity profile, with disturbances corresponding to an intensity of $15 \%$, was imposed at the inlet. The disturbance at the inlet was adapted to match laser Doppler anemometry data of the flow in a comparable phantom [129]. The cell density of the numerical mesh used for the CFD simulations was increasing when approaching the wall, thus avoiding errors associated with tracking particles close to the wall [130]. The temporal resolution of the LES simulation was $50 \mu \mathrm{s}$ and the smallest and largest cell volumes were $9.7 \cdot 10^{-11}$ and $2.9 \cdot 10^{-14} \mathrm{~m}^{3}$, respectively.

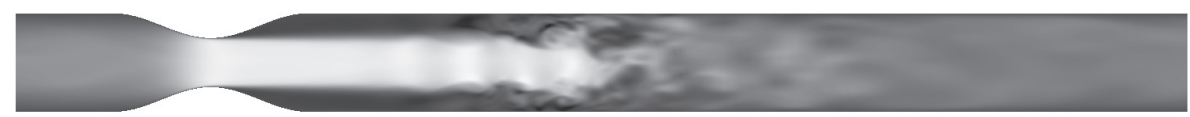

Figure 5. A snapshot of the flow in the stenotic phantom from the CFD data. The image is color coded according to the magnitude of velocity.

\subsubsection{Eulerian-Lagrangian PC-MRI simulation}

For a fixed reference in a moving fluid, the Bloch equation can be written as

$$
\frac{\mathrm{d} \boldsymbol{M}}{\mathrm{d} t}+\boldsymbol{V} \cdot \nabla \boldsymbol{M}=\gamma \boldsymbol{M} \times \boldsymbol{B}+-\frac{M_{x}}{T 2} \hat{x}-\frac{M_{y}}{T 2} \hat{y}-\frac{M_{z}-M_{0}}{T 1} \hat{z}
$$

For a frame of reference moving along a particle's trajectory, $\boldsymbol{V}=0$. The magnetic field, $\boldsymbol{B}$, can be separated into the external magnetic field, $\boldsymbol{B}_{0}$, the magnetic field produced by the radio frequency pulse, $\boldsymbol{B}_{1}$, and the magnetic gradient field, $\boldsymbol{B}_{\mathrm{g}}$. For a frame of reference moving along a particle's trajectory and rotating with the Larmor frequency, $\gamma \boldsymbol{B}_{0}=0$, and the Bloch equation can be expressed as

$$
\frac{\mathrm{d} \boldsymbol{M}}{\mathrm{d} t}=\gamma \boldsymbol{M} \times\left(\boldsymbol{B}_{\mathbf{1}}+\boldsymbol{B}_{\boldsymbol{g}}\right)-\frac{M_{x}}{T 2} \hat{x}-\frac{M_{y}}{T 2} \hat{y}-\frac{M_{\mathbf{z}}-M_{0}}{T 1} \hat{z} .
$$

Spin particle trajectories were calculated from the CFD data and Equation 7.2 was numerically solved for every spin separately using a fourth order Runge-Kutta algorithm. The magnetic gradient fields, $\boldsymbol{B}_{\mathrm{g}}$ and $\boldsymbol{B}_{1}$, were extracted from the MRI scanner software, facilitating comparison with measurements using identical pulse sequences and evaluation of current pulse sequences. The simulated PC-MRI signal was calculated as the complex sum of 
the transverse magnetization, $\Sigma\left(M_{\mathrm{x}}+\mathrm{i} M_{\mathrm{y}}\right)$. To obtain accurate IVSD estimates from the simulations, 500 spins per voxel were emitted in the region of interest.

\subsubsection{Validation and results}

For validation, velocity and IVSD estimates from the simulated PC-MRI measurements were compared to in-vitro PC-MRI measurements obtained using identical pulse sequences and an identical phantom. A $63 \%$ glycerol and $37 \%$ water solution at a temperature of $33^{\circ} \mathrm{C}$ with the same viscosity as the simulated fluid was used to mimic blood. A spin-warp pulse sequence was used and imaging parameters for the measurements and simulations are shown in Table 1. The VENC was optimized for IVSD mapping, and the velocity was manually corrected for velocity aliasing.

Table 1. Imaging parameters.

\begin{tabular}{llllllll}
\hline Orientation & TE $[\mathrm{ms}]$ & TR $[\mathrm{ms}]$ & $\begin{array}{l}\text { Flip } \\
\text { angle }\end{array}$ & $\begin{array}{l}\text { VENC } \\
{[\mathrm{m} / \mathrm{s}]}\end{array}$ & $\begin{array}{l}\text { Voxel size } \\
{\left[\mathrm{mm}^{3}\right]}\end{array}$ & FOV $\left[\mathrm{mm}^{3}\right]$ & NSA \\
\hline Frequency & 3.2 & 5.7 & $15^{\circ}$ & 1.5 & $2 \times 2 \times 2$ & $60 \times 158 \times 220$ & 8 \\
Slice & 3.2 & 5.7 & $15^{\circ}$ & 1.5 & $2 \times 2 \times 2$ & $220 \times 150 \times 130$ & 5 \\
\hline
\end{tabular}

Orientation describes the encoding in the principal flow direction. NSA is the number of signal averages.

The velocity and IVSD along the centerline of the phantom showed good agreement between the simulation, measurement and CFD data (Figure 6). Artifacts such as signal drop, intravoxel phase dispersion, ghosting and displacement, appeared both in the simulation and the measurements. Cross-sectional images of IVSD from the PC-MRI simulation, CFD data and in-vitro measurement can be seen in Figure 7. As expected, ghosting artifacts were only observed in the slice-encoding direction, resulting in less smooth IVSD curves. 

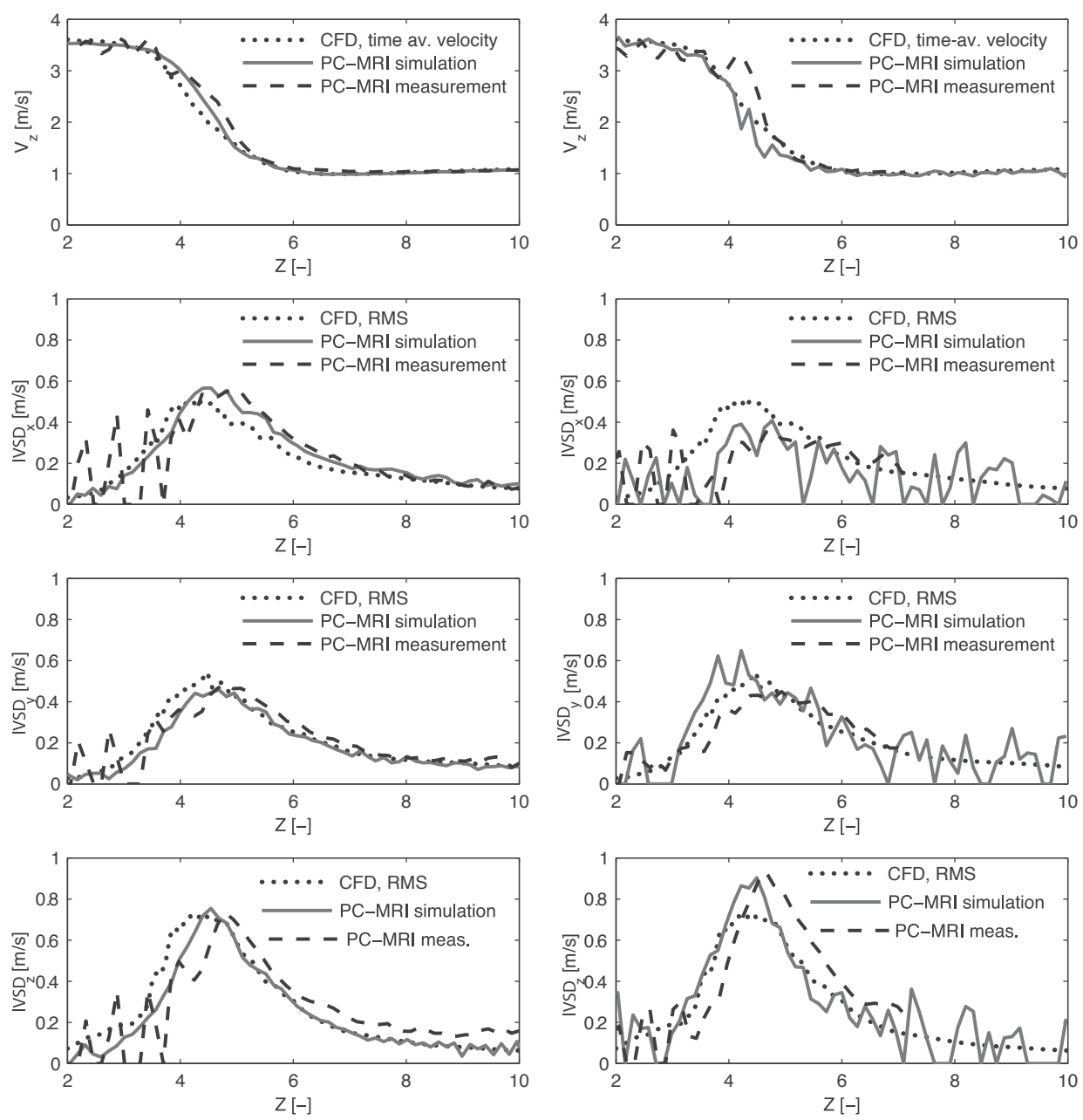

Figure 6. The mean velocity in the principal flow direction (z) and IVSD in all three directions along the centerline of the phantom from the PC-MRI simulations and PC-MRI measurements as well as the time averaged velocity and root means squares (RMS) of the fluctuating velocities from CFD. Left column: frequency-encoding in the principal flow direction. Right column: slice-encoding in the principal flow direction. $Z$ is the distance from the center of the stenosis, normalized by the unconstricted pipe diameter 

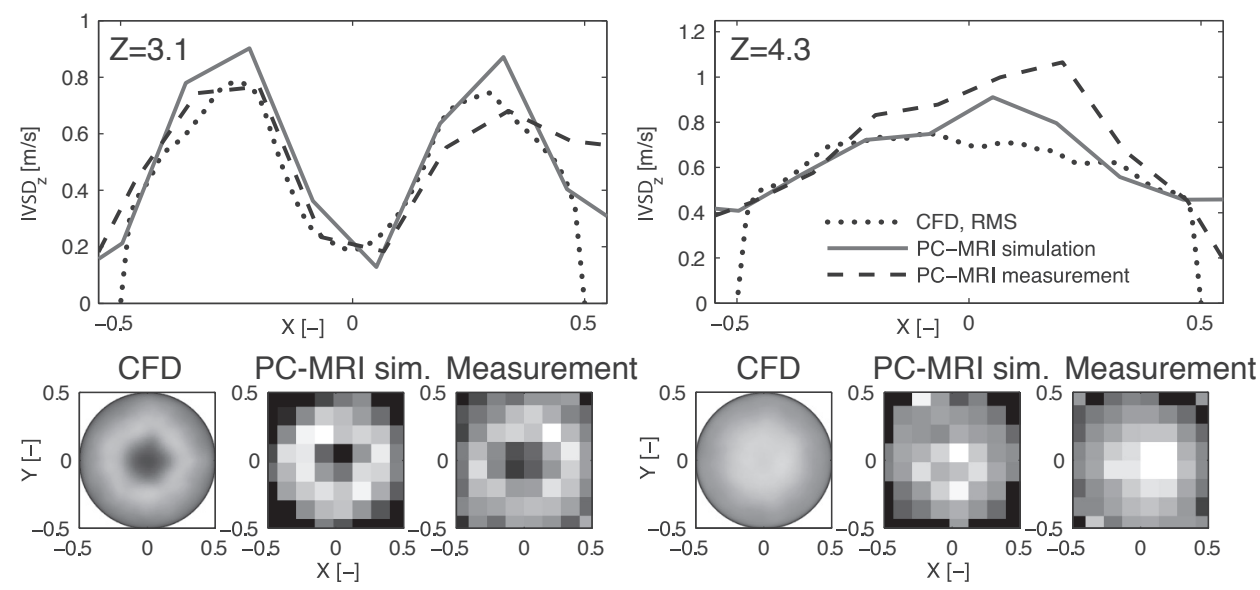

Figure 7. Cross sectional images of IVSD in the $z$-direction at the reattachment zone (Left: $Z=3.1$ ) and peak IVSD (Right: $Z=4.3$ ). Slice encoding was placed in the principal flow direction (Zdirection) to avoid displacement compared to CFD.

\subsection{Simulation of the intravoxel velocity distribution}

For simulations of a large number of flows and MRI parameter settings, the computational burden of the Eulerian and Eulerian-Lagrangian simulation approaches may be prohibitive. It is not always necessary to solve the Bloch equations in order to simulate PC-MRI measurements or a specific behavior of PC-MRI-based estimation of different parameters. Simplifications can be made to obtain less computationally expensive simulations and, for example, simulate the behavior of an isolated artifact. For example, it is possible to simulate a point-spread function (PSF) and convolve it with the flow field to obtain mean velocity estimates. This approach can be extended to acquire the intravoxel velocity distribution, $s(v)$, from the velocity field. The Fourier transform of $s(v)$ can be computed to obtain the MRI signal as a function of first order motion sensitivity, $S\left(k_{v}\right)$. As described in Chapter 4 , the PCMRI velocity and IVSD estimates can be obtained from the phase and magnitude of $S\left(k_{v}\right)$, respectively. This approach was used in Paper II to evaluate the accuracy of different MRIbased methods for WSS estimation.

In a $2 \mathrm{D}$ measurement, the $\mathrm{k}$-space is truncated by a square for Cartesian acquisition and a circle for spiral or radial acquisition. The PSF can be obtained by taking the inverse Fourier transform of this truncation window. In the Cartesian case, the PSF can be expressed as $\operatorname{sinc}(x / \Delta x) \cdot \operatorname{sinc}(y / \Delta y)$ (Figure 8 a), where $x$ and $y$ are the spatial coordinates and $\Delta x$ and $\Delta y$ describe the size of the voxel. Using spiral trajectories, the PSF can be expressed as jinc $\left(\sqrt{x^{2}+y^{2}} / \Delta r\right.$ ) (Figure $8 \mathrm{~b}$ ), where $\Delta r$ is the isotropic voxel size. Reconstruction and post-processing steps, such as Gibbs-artifact filtering, will also affect the PSF.

For example, this approach has been used to evaluate the accuracy of WSS estimates from 2D FVE measurements [96], and to evaluate the accuracy of PC-MRI turbulence mapping [65]. Gibbs ringing, velocity aliasing, partial volume artifacts and phase dispersion are also simulated using this approach. However, artifacts such as displacement and ghosting will not be present in the simulated data. This approach is very suitable for investigating different VENCs or multi-VENC techniques, as it is possible to obtain the signal for all possible 
VENCs from $S\left(k_{v}\right)$. If using a more advanced MRI simulation, such as the EulerianLagrangian approach, the different VENCs result in different gradients and a new simulation is necessary for every VENC.
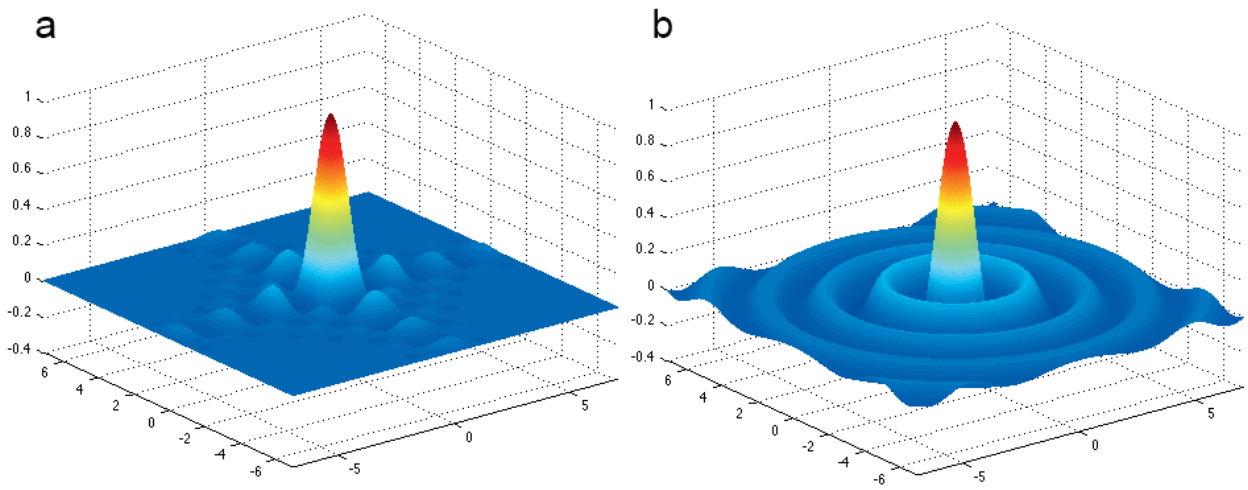

Figure 8. The PSF from a) a Cartesian (sinc) and b) a spiral acquisition (jinc). 


\section{Accuracy of MRI-based wall shear stress}

\section{estimation}

In Paper II, the accuracy of different WSS approaches and the impact of different parameter settings were investigated by using numerical simulations. The PC-MRI simulation approach based on simulation of the intravoxel velocity distribution, which was described in Section 7.2, was chosen to enable investigation of a large number of parameters and flow settings.

The accuracy was evaluated for five different approaches for MRI WSS estimation: linear extrapolation of PC-MRI velocity data (Vel-LE method), linear interpolation and wall segmentation (Vel-Wall method), parabolic fitting and wall segmentation (Vel-Parabolic method), FVE-based and IVSD-based. These methods were chosen to cover the basic principles of the methods presented in Section 4.6. The impact of spatial resolution, wall segmentation errors, VENC and partial volume artifacts was investigated. Furthermore, the simulations were carried out for a large range of WSS values $\left(1-20 \mathrm{~N} / \mathrm{m}^{2}\right)$ to investigate if WSS estimates are monotonically related to the actual WSS.

\subsection{Numerical flow data}

The numerical flow data consisted of twenty different high-resolution two-dimensional symmetric velocity fields in a circular tube with a diameter of $18 \mathrm{~mm}$ with WSS values ranging from 1-20 N/m $\mathrm{m}^{2}$. The range of WSS values was chosen to match the expected WSS values in the human aorta and carotid arteries as obtained by CFD simulations [83-85, 98-99]. The velocity profiles are shown in Figure 9 and consisted of a parabolic profile with analytically known WSS values by the wall and a plateau when the parabolic profile reached $1.5 \mathrm{~m} / \mathrm{s}$ to avoid unrealistic peak velocities.

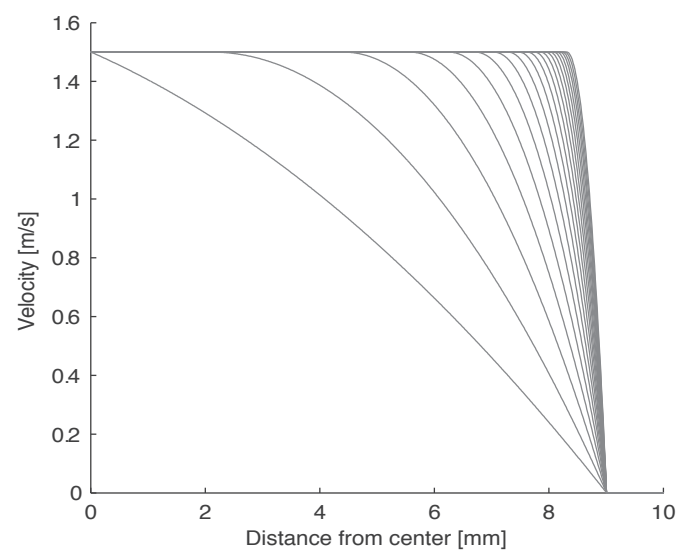

Figure 9. a) Plots of the 20 different velocity profiles considered, with WSS ranging from 1 to 20 $\mathrm{N} / \mathrm{m}^{2}$. The vessel diameter is $18 \mathrm{~mm}$. 


\subsection{WSS Estimation}

In all the methods described in this section, the WSS was estimated by multiplying the estimated spatial velocity derivative at the wall with the dynamic viscosity of blood, which was set to $4.6 \cdot 10^{-3} \mathrm{Ns} / \mathrm{m}^{2}$ in this work.

\subsubsection{Linear extrapolation}

Using the linear extrapolation approach (Vel-LE method), WSS was estimated from the velocity estimates from a voxel at the wall, $u_{1}$, and the adjacent voxel, $u_{2}$, in the direction of the inward normal of the wall. The spatial velocity derivative was estimated as the velocity difference divided by the distance between the voxel centers, $\Delta x$, i.e. the spatial resolution (Figure 11 a).

$$
\frac{\delta u}{\delta x}=\frac{u_{2}-u_{1}}{\Delta x}
$$

In order to vary the amount of partial volume artifacts, the voxel grid was adjusted stepwise so that the distance from the center of the wall voxel and the wall (lumen-wall interface) was varied between 0 and 1 voxel lengths (Figure 10).
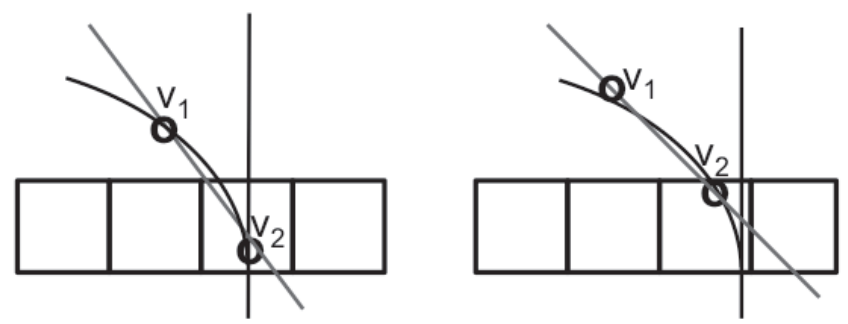

Figure 10. An illustration of how the position of the voxel grid relative the wall and the amount of partial volume may affect the WSS estimates of the LE approach.

\subsubsection{Linear interpolation and segmentation of the vessel wall}

The position of the wall may be estimated on sub-voxel level by segmentation or by estimation of the amount of partial volume [87]. For the Vel-Wall approach, we assumed that the position of the wall to be known with certain accuracy and the error of the wall position was modeled as a normal distribution with a standard deviation of $1 / 4$ voxel size. Linear interpolation was used to obtain the velocity value, $u_{\text {interp }}$, at a distance of one voxel length, $\Delta x$, from the wall in the direction of the inward normal (Figure $11 \mathrm{~b}$ ). The velocity at the estimated wall position was assumed to be zero and the spatial velocity derivative was obtained as

$$
\frac{\delta u}{\delta x}=\frac{u_{\text {interp }}}{\Delta x}
$$

The voxel grid was varied in the same way as in the LE approach. 


\subsubsection{Parabolic fitting and segmentation of the wall}

In order to represent the methods using more degrees of freedom, a parabolic function was fitted to the position of the wall and the velocity of the two voxels closest to the wall (VelParabolic method). Voxels with their center closer than half a voxel length from the wall were excluded from the parabolic fitting to obtain estimates less sensitive to segmentation errors. The error of the wall estimation was modeled as a normal distribution with a standard deviation of $1 / 4$ voxel size. Theoretically, velocity should be zero at the wall according to the no-slip condition, but forcing the velocity to zero at the estimated wall position also make the WSS estimates more sensitive to segmentation errors. Therefore, two versions of the parabolic approach were studied, one assuming zero velocity at the wall (Vel-Parabolic1, Figure $11 \mathrm{c}$ ) and a second using an interpolated velocity value at the wall (Vel-Parabolic2, Figure $11 \mathrm{~d}$ ). The voxel grid was varied in the same way as in the LE approach.

\subsubsection{Fourier velocity encoding-based method}

As described in Section 4.6, an intravoxel velocity profile can be estimated from the intravoxel velocity distribution as obtained by a FVE measurement [95-96]. A sampling function defined as $\operatorname{sinc}(u / \Delta u)$ was used to sample $s(u)$, simulating a FVE measurement with a velocity resolution of $\Delta u$. From the $s(u)$ samples, the intravoxel velocity profile, $r(u)$, was computed as

$$
\begin{aligned}
& r\left(u_{i}\right)=r\left(u_{i-1}\right)+\Delta r \cdot h\left(u_{i}\right), \\
& \text { where, } h\left(u_{i}\right)=\frac{\left|s\left(u_{i}\right)\right|}{\sum_{j}\left|s\left(u_{j}\right)\right|}
\end{aligned}
$$

$\Delta r$ is the spatial resolution and $r$ is the intravoxel spatial position. The spatial velocity derivative is then estimated by fitting a straight line in a velocity interval, $\left[u_{0} u_{1}\right]$ (Figure 11 e). The interval was set to $u_{0}=\Delta u \mathrm{~m} / \mathrm{s}$ and $u_{1}=4 \Delta u \mathrm{~m} / \mathrm{s}$. WSS estimates using the FVEbased approach were only computed for voxels containing both lumen and wall, and the voxel grid was varied so that the position of the voxel center was between $1 / 2$ voxel length into the lumen and $1 / 2$ voxel length into the wall.

\subsubsection{Intravoxel velocity standard deviation mapping-based method}

It has previously been suggested that the IVSD could be used to estimate WSS [97]. The presence of a velocity gradient in the voxel will result in phase dispersion and a relative signal loss that can be used to estimate the IVSD. The influence of WSS on the MRI signal, $S\left(k_{v}\right)$, is illustrated in Figure $11 \mathrm{f}$. This approach was extended in Paper I to obtain an estimate of the WSS from the IVSD. The IVSD is defined as the square root of the second central moment of the intravoxel velocity distribution.

$$
\operatorname{IVSD}=\sqrt{\int_{-\infty}^{\infty}(u-\bar{u})^{2} s(u) d u}[\mathrm{~m} / \mathrm{s}]
$$

The velocity gradient covered by the voxel was assumed to be linear and one-directional, resulting in a uniform intravoxel velocity distribution. Thus, measured intravoxel velocity distribution will have the shape of a one-dimensional projection of the PSF. By applying a Gaussian k-space filter, the PSF will be Gaussian. The previously described relationship between the IVSD and the signal magnitude of the PC-MRI measurement (Section 4.2) is based on the assumption of a Gaussian velocity distribution and is consequently also suitable for the estimation of the IVSD in the presence of a linear velocity gradient. With the assumption of the linear velocity profile, the velocity, $u$, in the voxel can be described as 


$$
u=\frac{\delta u}{\delta x} \cdot x+\bar{u}
$$

where $x$ is the spatial coordinate in the direction of the gradient and $x=0$ corresponds to the center of the voxel. From Equation 8.6, $x$ can be described as $x=\frac{(u-\bar{u})}{(\delta u / \delta x)}$ and the onedimensional projection of the PSF, $p s f(x)=p s f\left(\frac{(u-\bar{u})}{(\delta u / \delta x)}\right)$. This function describes the PSFweighted velocity distribution of the voxel, i.e. the measured intravoxel velocity distribution, $s(u)=\operatorname{ps} f\left(\frac{(u-\bar{u})}{(\delta u / \delta x)}\right)$. The spatial velocity derivative can be obtained by dividing the second central moment of $s(u)$ with the second moment of the $p s f(x)$.

$$
\frac{I V S D^{2}}{\int_{-\infty}^{\infty} x^{2} p s f(x) d x}=\frac{\int_{-\infty}^{\infty}(u-\bar{u})^{2} s(u) d u}{\int_{-\infty}^{\infty} x^{2} p s f(x) d x}=\frac{\int_{-\infty}^{\infty}(u-\bar{u})^{2} s(u) d u}{\int_{-\infty}^{\infty}\left(\frac{(u-\bar{u})}{(\delta u / \delta x)}\right)^{2} p s f\left(\frac{(u-\bar{u})}{(\delta u / \delta x)}\right) d u}=(\delta u / \delta x)^{2}
$$

and thus

$$
|(\delta u / \delta x)|=\sqrt{\frac{\mathrm{IVSD}^{2}}{\int_{-\infty}^{\infty} x^{2} p s f(x) d x}}
$$

The second moment of $p s f(x)$ was computed by numerical integration of a high-resolution representation of $\operatorname{ps} f(x)$. The spatial velocity derivate was estimated for two adjacent voxles, and the highest value was used to estimate the WSS. The voxel grid was varied so that the position of the innermost voxel was varied in the same interval as in the FVE-based method.

\subsection{MRI Simulations}

The PSF for a spatial resolution of $\Delta r$ was defined as jinc $\left(\sqrt{x^{2}+y^{2}} / \Delta r\right)$ for all methods except the IVSD-based, for which a Gaussian PSF was used. Thus, all PSF were symmetric, corresponding to a radial or spiral acquisition. From the high-resolution 2D velocity profiles, the PSF was used to compute the intravoxel velocity distribution, $s(v)$, for a row of voxels along the inward normal of the vessel. The MRI signal $S\left(k_{v}\right)$ was obtained by taking the Fourier transform of $s(v)$. The simulations did not incorporate noise and saturation effects. For the PC-MRI velocity-based methods (Vel-LE, Vel-Wall, Vel-Parabolic1-2) a symmetric encoding scheme was used to obtain $S\left(-k_{v} / 2\right)$ and $S\left(k_{v} / 2\right)$. The velocity was then computed as

$$
U=\frac{\operatorname{VENC}}{\pi}\left(\operatorname{angle}\left(S\left(k_{v} / 2\right)\right)-\operatorname{angle}\left(S\left(-k_{v} / 2\right)\right)\right)
$$

In order to obtain IVSD estimates, a reference scan segment with no velocity encoding is necessary and, therefore, a non-symmetric encoding scheme was used to obtain $S(0)$ and $S\left(k_{v}\right)$. The IVSD was then computed according to Equation 4.8. The FVE measurements were simulated by sampling $s(v)$ with a sampling function, $\operatorname{sinc}(u / \Delta u)$, using different velocity resolutions, $\Delta u$, to cover a velocity-encoding interval of $\pm 1.5 \mathrm{~m} / \mathrm{s}$. 

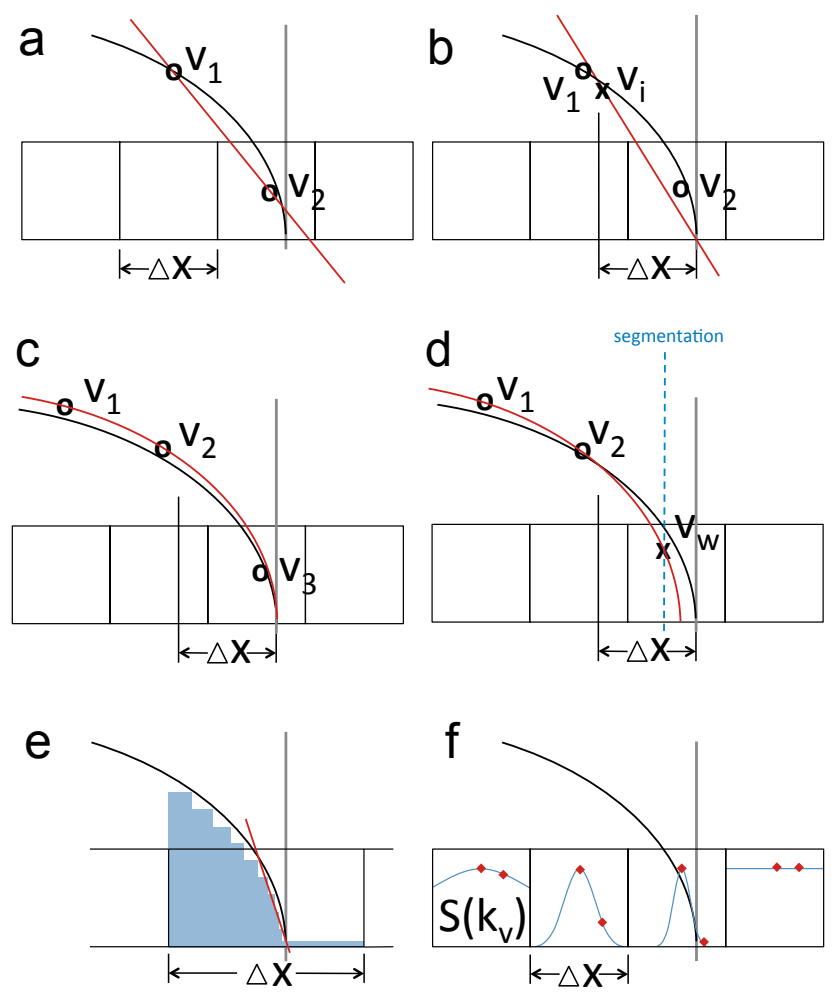

Figure 11. A schematic description of the different MRI-based WSS estimation methods applied in this work: a) Vel-LE, b) Vel-Wall, c) Vel-Parabolic1, d) Vel-Parabolic2, e) FVE and f) IVSD. The vertical grey line represents the vessel wall, the curved black line represents the velocity profile. The red line represents the estimated gradient or profile. The dashed blue line represents the estimated wall position (segmentation). $v_{1-3}$ are the PC-MRI voxel mean velocity values, $v_{i}$ and $v_{w}$ are velocity values interpolated one voxel length into the lumen and at the wall, respectively.

In order to investigate the effect of partial volume and voxel position relative to the wall, the voxel grid was shifted in the direction of wall's normal in ten uniformly distributed steps covering one voxel length. Thus, the complete range of voxel grid positions in the direction of the wall's normal were covered. The different ranges used for the WSS estimation approaches are described in Section 8.2 and reflect the voxels that each method is adapted for.

Isotropic voxel sizes of $0.5,1.0$ and $1.5 \mathrm{~mm}$ were considered in the PC-MRI simulations, corresponding to 36,18 and 12 voxels across a diameter of $18 \mathrm{~mm}$, respectively. Due to the time-consuming nature of the FVE measurements, larger voxels were used for the simulation of FVE. Voxel sizes of 3,4 and $5 \mathrm{~mm}$ were considered for the FVE simulations, corresponding to $6,4.5$ and 3.6 voxels across a diameter of $18 \mathrm{~mm}$, respectively. Smaller voxels would have required an increased velocity resolution, further increasing the scan time.

Three different VENC were considered for the PC-MRI simulations, 1,2 and $3 \mathrm{~m} / \mathrm{s}$. The velocity resolutions considered for the FVE simulation were $0.1,0.15$ and $0.2 \mathrm{~m} / \mathrm{s}$. The parameters used for the different simulations are summarized in Table 2. 
Table 2. Scan simulation parameters.

\begin{tabular}{lllll}
\hline Method & Voxel size, $\Delta \boldsymbol{r}[\mathrm{mm}]$ & VENC $[\mathrm{m} / \mathrm{s}]$ & $\Delta \boldsymbol{v}[\mathrm{m} / \mathrm{s}]$ & Voxel pos. interval \\
\hline Vel-LE & $0.5,1.0,1.5$ & $1,2,3$ & - & {$[01] \Delta r$} \\
Vel-Wall & $0.5,1.0,1.5$ & $1,2,3$ & - & {$[01] \Delta r$} \\
Parabolic1-2 & $0.5,1.0,1.5$ & $1,2,3$ & - & {$[01] \Delta r$} \\
FVE & $3,4,5$ & - & $0.1,0.15,0.2$ & {$[-.5 .5] \Delta r$} \\
IVSD & $0.5,1.0,1.5$ & $1,2,3$ & - & {$[-.5 .5] \Delta r$} \\
\hline
\end{tabular}

For the FVE method, a velocity interval of $[-1.51 .5] \mathrm{m} / \mathrm{s}$ was sampled with velocity resolution, $\Delta v$. The voxel position is the position relative to the wall of the center of the outermost voxel included in WSS estimation; positive values are in the lumen.

\subsection{Results and interpretation}

There was generally a very weak linear relationship between estimated and actual WSS for all methods in the interval $0-20 \mathrm{~N} / \mathrm{m}^{2}$. While the Vel-Parabolic1 method for a voxel size of 0.5 mm showed a moderate linear relationship $\left(\beta=0.95, \mathrm{R}^{2}=0.53\right)$ in the $0-20 \mathrm{~N} / \mathrm{m}^{2}$ interval, this configuration showed less good performance in the $0-5 \mathrm{~N} / \mathrm{m}^{2}$ interval, where WSS was overestimated $\left(\beta=1.63, \mathrm{R}^{2}=0.26\right)$. For the Vel-Wall method, a moderate relationship was found in the $0-5 \mathrm{~N} / \mathrm{m}^{2}$ interval for $1 \mathrm{~mm}$ voxels $\left(\beta=0.56, \mathrm{R}^{2}=0.90\right)$.

For all methods, higher spatial resolution generally resulted in higher WSS estimates, but not necessarily more accurate estimates. As expected, the voxel size had a considerable influence on the estimated WSS, especially for the velocity-based methods, and the influence increased with higher WSS.

The two linear methods, Vel-LE and LE-wall underestimated high WSS (Figure 12 a-b). However, the Vel-Wall method using a voxel size of $0.5 \mathrm{~mm}$ overestimated WSS in the $0-5$ $\mathrm{N} / \mathrm{m}^{2}$ interval. Some influence of the voxel position was observed for the Vel-LE method for high WSS. The Vel-Wall method was less sensitive to voxel position relative to the wall, but some sensitivity for segmentation errors was found for high WSS.

The Vel-Parabolic1 method was able to resolve higher WSS values than the linear methods (Figure $12 \mathrm{c}$ ), but was sensitive to segmentation errors. The Vel-Parabolic2 method was less sensitive to segmentation errors for WSS but did not resolve as high WSS values (Figure 12 d). For high WSS, the estimates from Vel-Parabolic2 even decreased with increasing WSS.

The FVE method was very sensitive to voxel position, and although the mean of the WSS estimates seems to agree fairly well with the true WSS, errors up to $300 \%$ were observed (Figure $12 \mathrm{e}$ ).

The accuracy of the IVSD-based method was similar to that of the Vel-LE method and, WSS was underestimated for all parameter settings and intervals (Figure $12 \mathrm{f}$ ). The IVSD was less sensitive to wall position than the Vel-LE method. 



Figure 12. The WSS estimated using a) the Vel-LE method, b) Vel-Wall method, c) Vel-Parabolic1 (no-slip assumed) d) Vel-Parabolic2. For a-d,f) the voxel sizes were $0.5,1,1.5 \mathrm{~mm}$ and the VENC was $2 \mathrm{~m} / \mathrm{s}$. For the FVE method d) the voxel size was 3, 4, $5 \mathrm{~mm}$ and the velocity resolution was $0.15 \mathrm{~m} / \mathrm{s}$. The estimated WSS is shown on the vertical axes and the analytically derived WSS on the horizontal axes. Error bars show the standard deviation due to voxel center position relative to the wall and segmentation error if applicable. The dotted line shows the identity line. 


\section{Spiral 4D flow}

As many cardiovascular applications of 4D flow MRI require large volumetric coverage, scan times of about 20-40 minutes are not unusual. These long scan times are one of the major limitations of 4D flow MRI and prohibit the extended use of the technique. Parallel imaging and similar techniques can reduce the number of k-space samples necessary at the cost of SNR, but an inherent problem of Cartesian spin-warp imaging is its low scan efficiency. Spiral k-space trajectories can cover a larger part of k-space during each TR and have previously been used for velocity mapping in applications requiring fast imaging, such as real-time flow imaging and breath-hold acquisitions [114-116].

In Paper III, a novel spiral 4D flow sequence using prospective cardiac gating was implemented and validated in-vivo for aortic flow by comparison with a conventional Cartesian sequence and 2D flow measurements. In Paper IV, the spiral 4D flow sequence was extended to retrospective cardiac gating and adapted for intra-cardiac 4D flow and validated in-vivo by comparison with a conventional Cartesian sequence and 2D flow measurements. In order to enable retrospective cardiac gating, offline reconstruction was necessary.

Spiral MRI sequences are inherently less sensitive to displacement and acceleration artifacts. In Paper V, the feasibility of spiral k-space trajectories for the measurement of stenotic flow was investigated by in-vitro measurements. Velocity and turbulence estimates from the spiral sequence were evaluated by comparison with a Cartesian sequence and CFD data.

\subsection{Stack-of-spirals 4D flow sequence}

This section describes the implemented spiral 4D flow sequence. An interleaved stack-ofspirals was used to traverse a 3D k-space. The use of interleaved spiral trajectories enables a higher temporal resolution and limits the effects of off-resonance. Cartesian spin-warp phase encoding was used to cover the k-space in the slice direction. This spatial phase encoding was carried out simultaneously with the velocity encoding in order to minimize the TE. A 1-1 spectral-spatial pulse designed to suppress the signal of fat was used for excitation of the volumetric slab in Paper III and IV. As the signal of fat is off-resonance, it will cause blurring of the image. Pilot experiments using the 1-1 pulse showed a significantly improved visual image quality compared to the standard RF pulse. While a 1-2-1 pulse would be more efficient in the suppression of fat, it would prolong the duration of the pulse and decrease the temporal resolution. The spiral 4D flow pulse sequence is illustrated in Figure 13. 


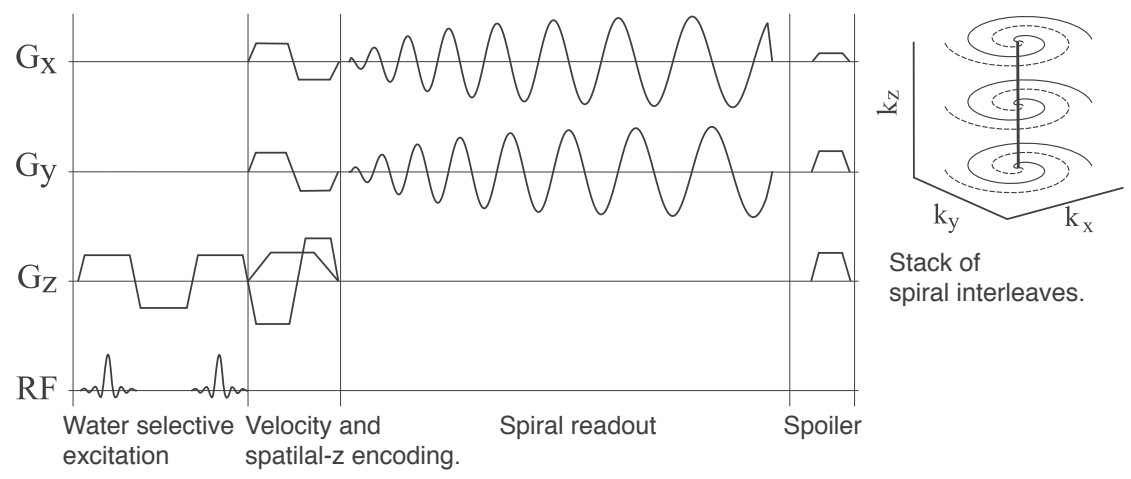

Figure 13. A pulse sequence diagram describing the interleaved stack-of-spiral pulse sequence. Velocity encoding was performed simultaneously as spatial encoding in the z-direction (slice direction).

The spiral pulse sequence was implemented in a clinical 1.5 T MRI system (Achieva; Philips) with a $33 \mathrm{mT} / \mathrm{m}$ gradient strength and $180 \mathrm{~T} / \mathrm{m} / \mathrm{s}$ slew rate. A five element cardiac SENSE coil was used for signal reception in all measurements.

\subsection{Prospectively-gated aortic 4D flow MRI}

In Paper III, the spiral pulse sequence described in Section 9.1 was used to reduce the scan time of prospectively-gated 4D flow imaging of the human aorta. Pathline analysis and cardiac output estimates were used to evaluate three different spiral 4D flow acquisitions invivo. The results of the spiral acquisition were compared to a conventional Cartesian 4D flow acquisition and a Cartesian 2D trough-plane velocity measurement in the ascending aorta. The SNR and background phase errors were studied using in-vitro measurements on a stationary phantom.

\subsubsection{Acquisition and reconstruction}

Ten healthy volunteers were imaged after they gave their written consent. None of the measurements were excluded from the study. Three different spiral sequences were used to image the aorta: 1) A TR-interleaved sequence with 12 spiral interleaves (TR-interleaved spiral); 2) A beat-interleaved sequence with 9 spiral interleaves (Beat-interleaved spiral); 3) A beat-interleaved sequence with 6 spiral interleaves (Short beat-interleaved). For comparison, a conventional Cartesian spin-warp 4D flow sequence accelerated using SENSE of factor two in the phase encoding direction (anterior-posterior) was acquired. The scan parameters for the 4D flow sequences are shown in Table 3. Additionally, a Cartesian retrospectively-gated 2D through-plane measurement was applied in the ascending aorta to obtain estimates of cardiac output. The following parameters were used for the 2D measurement: TE $2.6 \mathrm{~ms}$, TR $4.8 \mathrm{~ms}$, FOV $320 \times 260 \mathrm{~mm}$, voxel size $2.5 \times 2.7 \mathrm{~mm}^{2}$, slice thickness $8 \mathrm{~mm}$, SENSE reduction factor 2 , VENC $200 \mathrm{~cm} / \mathrm{s}, 4 k$-space lines per cardiac cycle. 
Table 3. Scan parameters for the 4D flow sequences.

\begin{tabular}{|c|c|c|c|c|}
\hline & $\begin{array}{l}\text { TR-interleaved } \\
\text { spiral }\end{array}$ & $\begin{array}{l}\text { Beat-interleaved } \\
\text { spiral }\end{array}$ & $\begin{array}{l}\text { Short beat- } \\
\text { interleaved spiral }\end{array}$ & $\begin{array}{l}\text { Cartesian 4D } \\
\text { flow }\end{array}$ \\
\hline TE/TR [ms] & $3.5 / 12.0$ & $3.5 / 16.0$ & $3.5 / 16.0$ & $3.4 / 6.1$ \\
\hline $\begin{array}{l}\text { Number of } \\
\text { interleaves }\end{array}$ & 12 & 9 & 6 & - \\
\hline $\begin{array}{l}\text { Readout } \\
\text { duration [ms] }\end{array}$ & 5.3 & 9.4 & 9.3 & 3.0 \\
\hline $\begin{array}{l}\text { Field of view } \\
{\left[\mathrm{mm}^{3}\right]}\end{array}$ & $260 \times 260 \times 45-50$ & $260 \times 260 \times 45-50$ & $260 \times 260 \times 45-50$ & $300 \times 300 \times 45-50$ \\
\hline $\begin{array}{l}\text { Voxel size } \\
{\left[\mathrm{mm}^{3}\right]}\end{array}$ & $2.8 \times 2.8 \times 2.8$ & $2.8 \times 2.8 \times 2.8$ & $2.8 \times 2.8 \times 2.8$ & $2.8 \times 2.8 \times 2.8$ \\
\hline $\begin{array}{l}\text { Temporal } \\
\text { resolution [ms] }\end{array}$ & 48.8 & 48.8 & 48.8 & 48.8 \\
\hline $\begin{array}{l}\text { Velocity } \\
\text { encoding } \\
{[\mathrm{cm} / \mathrm{s}]}\end{array}$ & 150 & 150 & 150 & 150 \\
\hline
\end{tabular}

In order to compare the SNR and background phase errors of the four 4D flow measurements, in-vitro measurements on a stationary phantom were performed. Only a single timeframe was acquired, and no respiratory or cardiac gating was simulated in the in-vitro measurement. Additional noise scans were obtained with all gradients and RF pulses turned off. The additional noise scan results in the doubling of scan time and was therefore not used in-vivo as the total duration of all scans was already around one hour.

Effects of concomitant gradient terms on the phase were automatically corrected for in the reconstruction [74]. Phase offsets due to eddy currents were corrected by subtraction of a second-order polynomial fitted to static tissue [75].

\subsubsection{Data analysis}

Volume flow estimation and pathline analysis are two common approaches to analysis and visualization of 4D flow MRI data. Pathline analysis requires high-quality velocity data as errors including noise and phase offsets are accumulated along the trajectory. Consequently, pathline analysis and cardiac output were used for quality assessment.

For comparison of cardiac output, the $4 \mathrm{D}$ flow data were reformatted to the plane of the $2 \mathrm{D}$ measurement in the ascending aorta. Two independent observers segmented the vessel using Segment [131], and the average cardiac output from the two observers was used for comparison.

The pathlines were emitted backwards from the descending aorta to exclude flow to the supraaortic branches. The time of emission was set to the downslope of the flow curve at the emission site. Pathlines leaving a segmentation of the thoracic aorta excluding the supra-aortic branches were counted as incorrect. Thus, the percentage of pathlines that resided within the segmentation was used as a quality measurement. Pathlines reaching the ascending aorta at the level of the pulmonary artery and the end of the segmentation were also counted as not having left the segmentation. 
As the pathline analysis described above focused on systolic flow, pathlines were also released from the entire segmentation during the four last timeframes to study the performance of the different sequences in diastolic flow. The number of traces that remained within the segmentations was computed as previously.

Background phase errors were computed for a region of interest in the stationary phantom before and after correction of phase offsets by phase subtraction. Spatially uniform noise images were reconstructed, and the magnitude images were divided by the standard deviations of the noise images to obtain SNR images [132]. The mean and standard deviation of the SNR in a segmentation of the phantom were reported in the results.

\subsubsection{Results and interpretation}

The mean scan time (including respiratory gating) was reduced by more than two-fold for all three spiral sequences compared to the Cartesian sequence, and for the short beat-interleaved sequence the scan time was reduced by more than three-fold (Table 4).

Table 4. Total scan times for the 4D flow acquisitions.

\begin{tabular}{lll}
\hline & Scan time & Relative to Cartesian \\
\hline Cartesian & $14 \mathrm{~m} 57 \mathrm{~s} \pm 4 \mathrm{~m} 36 \mathrm{~s}$ & $100 \%$ \\
TR-interleaved spiral & $6 \mathrm{~m} 12 \mathrm{~s} \pm 1 \mathrm{~m} 16 \mathrm{~s}$ & $43 \pm 9 \%$ \\
Beat-interleaved spiral & $6 \mathrm{~m} 21 \mathrm{~s} \pm 1 \mathrm{~m} 32 \mathrm{~s}$ & $44 \pm 10 \%$ \\
Short beat-interleaved spiral & $4 \mathrm{~m} 04 \mathrm{~s} \pm 1 \mathrm{~m} 08 \mathrm{~s}$ & $28 \pm 5 \%$ \\
\hline
\end{tabular}

The numbers are given as mean $\pm 1 S D$ for $n=10$. The average navigator efficiency was $64 \pm 10 \%$. The scan time is the total scan time including respiratory navigator.

No significant difference in cardiac output was found between the four 4D flow sequences (Table 5). However, there were significant differences between the 2D measurement and the two beat-interleaved spiral acquisitions. No significant difference was found for cardiac output by the two observers except for the $2 \mathrm{D}$ measurement, where there was a mean difference of $0.28 \pm 0.26 \mathrm{~L} / \mathrm{min}(\mathrm{P}<0.008$, paired t-test with Bonferroni correction). The Bland-Altman analysis showed a negative bias for the spiral acquisitions compared to the mean of all five acquisitions (Paper III). Again, the beat-interleaved sequences seem slightly less accurate than the TR-interleaved. The 2D measurement yielded the highest mean cardiac output, which may be attributed to its higher temporal resolution.

No significant differences in the systolic pathlines were found, but for the diastolic pathlines the number of pathlines that remained in the segmentation was significantly higher than for the Cartesian and TR-interleaved sequences compared to the beat-interleaved methods (Table 5). Visual inspection of the pathlines did not reveal any apparent differences between the four different acquisitions. As an example, Figure 14 shows pathlines from all four 4D flow acquisitions from a single volunteer. Similar results regarding the fraction of pathlines that remained within the segmentation have been reported previously [133]. Although no significant differences were found, the percentage of pathlines within the segmentation was slightly higher for the TR-interleaved spiral sequence as compared to the Cartesian. The beatinterleaved sequences performed slightly less well for the diastolic flow, which may be related to higher sensitivity to beat-to-beat variations. 
Table 5. Cardiac output and pathlines analysis.

\begin{tabular}{llll}
\hline & $\begin{array}{l}\text { Cardiac output } \\
\text { [L/min] }\end{array}$ & $\begin{array}{l}\text { Systolic pathlines } \\
\text { within segmentation } \\
(\%)\end{array}$ & $\begin{array}{l}\text { Diastolic pathlines } \\
\text { within segmentation } \\
(\%)\end{array}$ \\
\hline Cartesian & $6.2 \pm 1.0$ & $51 \pm 16 \%$ & $64 \pm 6 \%$ \\
TR-interleaved spiral & $5.8 \pm 1.0$ & $60 \pm 7 \%$ & $65 \pm 3 \%$ \\
Beat-interleaved spiral & $5.7 \pm 1.1^{*}$ & $52 \pm 10 \%$ & $57 \pm 5 \%$ \\
$\begin{array}{l}\text { Short-beat-interleaved } \\
\text { spiral }\end{array}$ & $5.6 \pm 0.8 *$ & $56 \pm 11 \%$ & $55 \pm 6 \%$ \\
2D through-plane & $6.4 \pm 1.6$ & & - \\
\hline
\end{tabular}

No statistically significant differences in cardiac output were found between any of the four 4D flow acquisitions. *: $\mathrm{p}<0.05$ versus $2 \mathrm{D}$ through-plane (two-way ANOVA, Tukey post-hoc test). No statistically significant differences were found between any methods for the systolic pathlines, but the number of diastolic pathlines within the segmentation for the Cartesian and TR interleaved spiral were significantly higher than for the beat and short beat interleaved spiral methods (two-way ANOVA, Tukey post-hoc test). The numbers are given as mean \pm 1 SD for $n=10$.

The background phase errors and SNR from the in-vitro measurements are shown in Table 6. Background errors before phase correction were slightly larger for the three spiral acquisitions, but these differences disappeared after phase corrections. The higher phase errors before correction in the spiral data are probably caused by the short distance between the bipolar gradient and the acquisition of the center of k-space. Short-term eddy currents caused by the bipolar gradients will then have a greater effect on the center of k-space compared to Cartesian sampling. Despite the reduction in scan time, the SNR was similar for all four acquisitions and the beat-interleaved spiral provided the highest SNR.

Table 6. Background phase errors and SNR in-vitro

\begin{tabular}{llll}
\hline & $\begin{array}{l}\text { Phase error before } \\
\text { correction }[\mathrm{m} / \mathrm{s}]\end{array}$ & $\begin{array}{l}\text { Phase error after } \\
\text { correction }[\mathrm{m} / \mathrm{s}]\end{array}$ & Mean SNR \\
\hline Cartesian & $-0.06 \pm 0.02$ & $0.00 \pm 0.02$ & $178 \pm 52$ \\
TR-interleaved spiral & $-0.18 \pm 0.03$ & $0.00 \pm 0.02$ & $155 \pm 51$ \\
Beat-interleaved spiral & $-0.16 \pm 0.03$ & $0.00 \pm 0.02$ & $206 \pm 70$ \\
$\begin{array}{l}\text { Short beat-interleaved } \\
\text { spiral }\end{array}$ & $-0.16 \pm 0.03$ & $0.00 \pm 0.02$ & $166 \pm 58$ \\
\hline
\end{tabular}

Phase errors before and after correction of velocity offsets by subtraction of a second-order polynomial. 


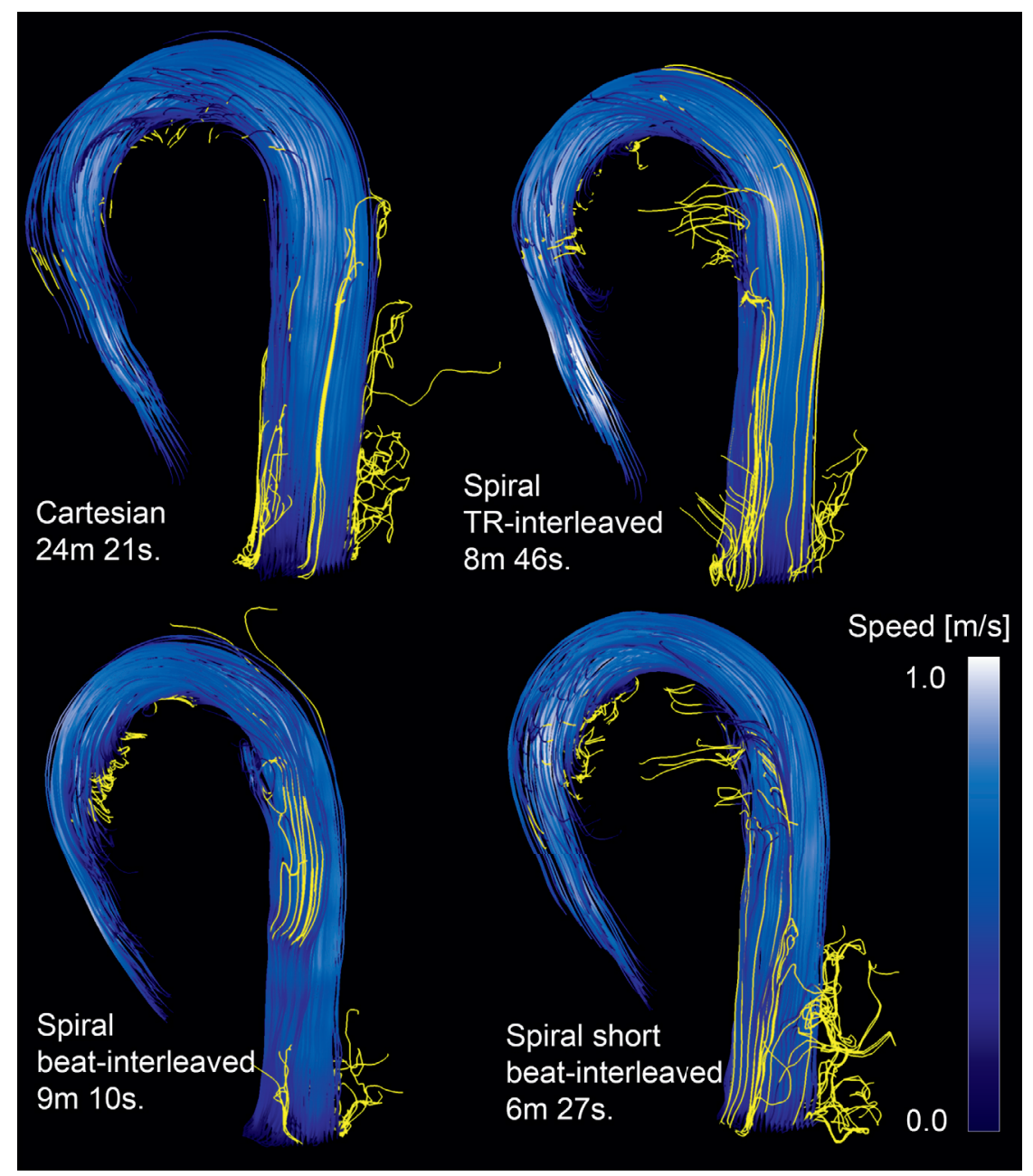

Figure 14. Pathlines for all four 4D flow acquisitions from a single volunteer. Pathlines were traced backwards from the descending aorta to avoid branching flow. Traces remaining within the aortic segmentation are colored according to speed, but are yellow where they exit the segmentation. 


\subsection{Retrospectively-gated intra-cardiac 4D flow MRI}

In Paper IV, the spiral 4D flow sequence used in Paper III was extended for retrospective cardiac gating by usage of an offline reconstruction algorithm capable of retrospective reconstruction of spiral data. This enables coverage of the complete cardiac cycle and a more comprehensive evaluation of the performance of spiral 4D flow for diastolic flow. The performance of the retrospective spiral 4D flow sequence was validated for volume flow and quantitative pathline analysis of intra-cardiac velocities over the complete heart cycle. The results were compared to conventional Cartesian 4D flow measurements and 2D throughplane velocity measurements in the aorta.

\subsubsection{In-vivo measurements}

Seven healthy volunteers and two patients were imaged after they gave their written consent. None of the measurements were excluded from the study. Patients with a stenosis or severe regurgitation were not considered in this study. Spiral retrospectively-gated 4D flow data and Cartesian 2D through-plane velocity measurements in the ascending aorta were obtained for all participants. Cartesian 4D flow data were only obtained for the healthy volunteers as the combined scan time of both 4D flow scans was considered too long for the patients. A singleoblique FOV was used for the 4D flow acquisitions to cover the entire left ventricle (LV) and the ascending aorta. The scan parameters for the 4D flow measurements are shown in Table 7.

The spiral data were reconstructed using ReconFrame (Gyrotools Inc., Zurich, Switzerland), as the in-scanner reconstruction software did not support reconstruction of retrospective spiral data. The Cartesian 4D and 2D data and bSSFP images were reconstructed on the scanner. Forty timeframes were retrospectively reconstructed for all flow data, using a non-linear approach for the retrospective reconstruction.

Table 7. Scan parameters.

\begin{tabular}{llll}
\hline & Spiral 4D & Cartesian 4D & Cartesian 2D \\
\hline $\begin{array}{l}\text { Echo time/TR }(\mathrm{ms}) \\
\begin{array}{l}\text { Number of } \\
\text { interleaves }\end{array}\end{array}$ & $3.7 / 12$ & $3.4 / 5.8$ & $2.9 / 4.8$ \\
$\begin{array}{l}\text { Readout Duration } \\
(\mathrm{ms})\end{array}$ & 5 & - & - \\
FOV $\left(\mathrm{mm}^{2}\right)$ & $280 \times 280 \times 101-112$ & $235-280 \times 280 \times 101-112$ & $320 \times 260$ \\
Voxel size $\left(\mathrm{mm}^{3}\right)$ & $2.8 \times 2.8 \times 28$ & $2.8 \times 2.8 \times 28$ & 2.1 \\
$\begin{array}{l}\text { Temporal resolution } \\
(\mathrm{ms})\end{array}$ & $48-48.8$ & $46.4-46.5$ & $2.5 \times 2.5 \times 8$ \\
$\begin{array}{l}\text { Velocity encoding } \\
(\mathrm{cm} / \mathrm{s})\end{array}$ & 120 & 120 & 48.4 \\
\hline
\end{tabular}




\subsubsection{Data analysis}

The net volume flow and peak flow rate in the segmentation of the ascending aorta were computed for all flow measurements using Segment [131]. A semi-automatic quantitative pathline analysis method tracing pathlines over the complete heart cycle was used to evaluate and compare the performance of the 4D flow sequences [70]. Pathlines were released backward and forward from a segmentation of the LV at end diastole (Figure 15). A segmentation of the LV at end systole was then used to determine if and where the pathlines left or entered the LV. Pathlines leaving the segmentation through the myocardium were counted as invalid. The valid pathlines were used to compute the inflow and the outflow from the LV. In accordance with the principle of mass conservation, the inflow and outflow should be equal. Consequently, the difference in inflow and outflow can be used as a quality measure of intra-cardiac velocity mapping.

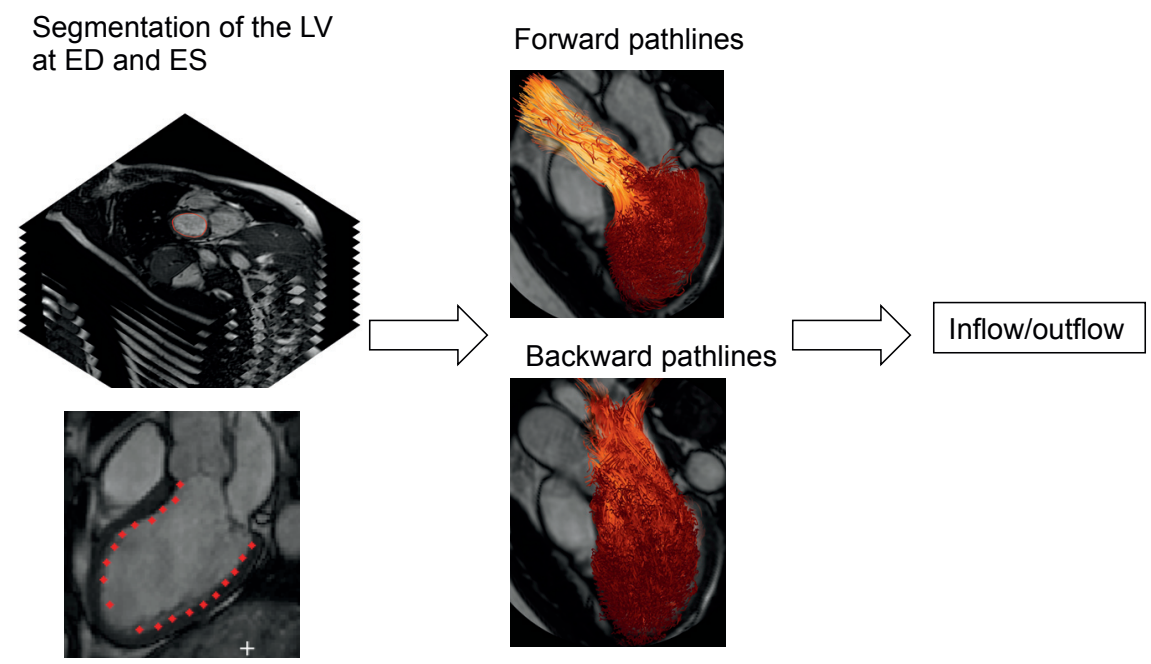

Figure 15. In the quantitative pathline analysis, pathlines are released backward and forward from a manual segmentation of the LV at end diastole (ED). A segmentation of the LV at end systole (ES) is then used to determine if and where the pathlines left the LV. Inflow and outflow are computed from the pathlines that left the LV through the top plane of the segmentation in the backward and forward analysis, respectively.

\subsubsection{Results and interpretation}

The mean scan time (including respiratory gating) of the spiral 4D flow measurements was $13 \pm 3$ min compared to $31 \pm 7 \mathrm{~min}$ for the Cartesian 4D flow measurements. This corresponds to more than a two-fold decrease in scan time compared to a scan already accelerated using SENSE factor 2.

There was generally good agreement between the volume flows and no significant differences between the net volume flow and peak flow rate from the three flow sequences were found (Table 8). 
Table 8. Aortic net volume flow and peak flow rate.

\begin{tabular}{lll}
\hline & Net volume flow $(\mathrm{ml}) *$ & Peak flow rate $(\mathrm{ml} / \mathbf{s}) \dagger$ \\
\hline 2D Aorta & $93 \pm 18$ & $490 \pm 101$ \\
4D Spiral & $89 \pm 14$ & $450 \pm 97$ \\
4D Cartesian & $93 \pm 11$ & $457 \pm 60$ \\
\hline
\end{tabular}

No significant differences were found between any of the acquisitions using a one-way ANOVA test $\left({ }^{*} \mathrm{p}=0.878, \dagger p=0.675\right)$. All values are represented as mean \pm one SD for $n=7$.

Although the quantitative pathline analysis revealed no significant difference between LV inflow and outflow for both the spiral and Cartesian 4D flow acquisitions (Table 9), a BlandAltman analysis of inflow vs. outflow for the spiral acquisition showed a slightly larger bias for the spiral 4D flow data (Paper IV). For spiral 4D flow, a bias of 8.7 and limits of agreement $\pm 18.9 \mathrm{ml}$ (Inflow-Outflow) were obtained, compared to a bias of 2.7 and limits of agreement $\pm 9.1 \mathrm{ml}$ for Cartesian 4D flow. The cause of this bias is difficult to pinpoint. Unsuccessfully corrected velocity offsets, noise or other artifacts may influence the pathlines. No significant differences were found when comparing the inflow, outflow and inflowoutflow from the spiral and Cartesian data. Visual inspection of the pathlines revealed no apparent differences between the spiral and Cartesian 4D flow acquisitions. As an example, the pathlines from two of the healthy volunteers are shown in Figure 16.

Table 9. Quantitative pathline analysis.

\begin{tabular}{llllll}
\hline & $\begin{array}{l}\text { Total scan } \\
\text { time }(\mathrm{min})\end{array}$ & $\begin{array}{l}\text { LV Inflow } \\
(\mathrm{ml})^{*}\end{array}$ & $\begin{array}{l}\text { LV Outflow } \\
(\mathrm{ml}) \dagger\end{array}$ & $\begin{array}{l}\text { IInflow- } \\
\text { outflowl }(\mathrm{ml}) \neq\end{array}$ & $\begin{array}{l}\text { p-value } \\
\text { Inflow vs. } \\
\text { Outflow }\end{array}$ \\
\hline 4D Spiral & $13 \pm 3$ & $93 \pm 17$ & $84 \pm 18$ & $8.8 \pm 9.5$ & 0.055 \\
4D Cartesian & $31 \pm 7$ & $88 \pm 15$ & $85 \pm 16$ & $4.1 \pm 3.3$ & 0.168 \\
\hline
\end{tabular}

No significant difference was found between inflow and outflow for the spiral $(p=0.055)$ and Cartesian $(p=0.168)$ measurements using two-tailed paired t-tests. No significant difference was found between the spiral and Cartesian flow values $\left({ }^{*} p=0.269, t p=0.734, \neq p=0.133\right)$. Volume flow values are represented as mean \pm one SD for $n=7$. 


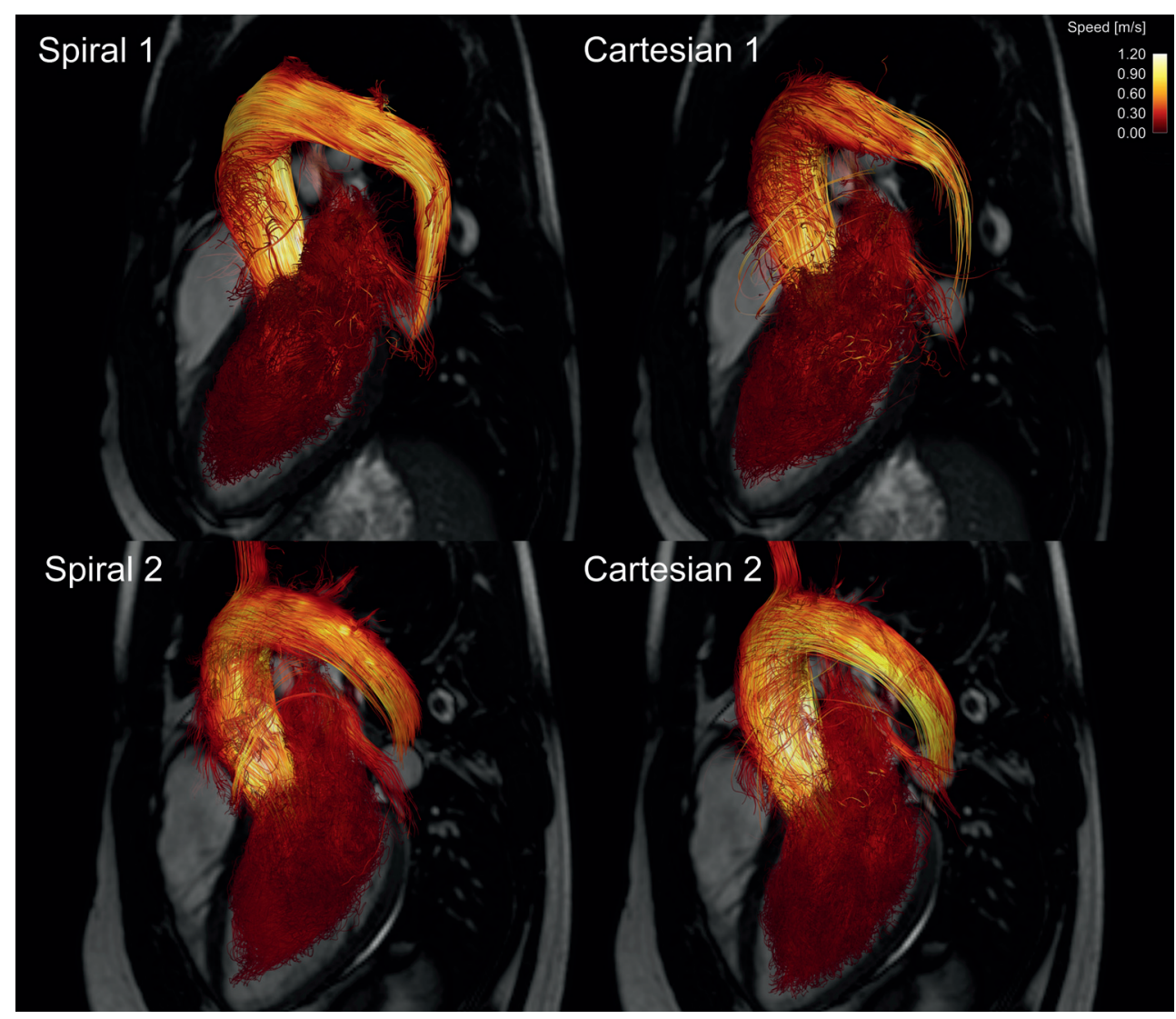

Figure 16. Pathlines covering the complete heart cycle from two of the healthy volunteers from the spiral and the Cartesian 4D flow measurements. Three-chamber bSSFP images are shown for orientation. Pathlines are color-coded according to speed.

The difference between inflow and outflow was slight for Patient 2 (Table 10). However, for Patient 1 the pathline outflow and aortic volume flow from the spiral 4D flow data were slightly lower compared to the pathline inflow. Some uncorrected velocity aliasing was observed in the ascending aorta of Patient 1 , which may have affected the pathline analysis. The pathlines from the two patients can be seen in Figure 17. 
Table 10. Patient pathline analysis and aortic flow comparison.

\begin{tabular}{llllllll}
\hline & \multicolumn{2}{l}{ Spiral 4D flow } & & & & 2D flow \\
& $\begin{array}{l}\text { Total } \\
\text { scan } \\
\text { time } \\
(\mathrm{min})\end{array}$ & $\begin{array}{l}\text { Inflow } \\
(\mathrm{ml})\end{array}$ & $\begin{array}{l}\text { Outflow } \\
(\mathrm{ml})\end{array}$ & $\begin{array}{l}\text { Aortic } \\
\text { flow }(\mathrm{ml})\end{array}$ & $\begin{array}{l}\text { Peak } \\
\text { flow rate } \\
(\mathrm{ml} / \mathrm{s})\end{array}$ & $\begin{array}{l}\text { Aortic } \\
\text { flow }(\mathrm{ml})\end{array}$ & $\begin{array}{l}\text { Peak } \\
\text { flow rate } \\
(\mathrm{ml} / \mathrm{s})\end{array}$ \\
\hline Patient 1 & 14 & 58 & 47 & 48 & 299 & 62 & 352 \\
Patient 2 & 15 & 56 & 54 & 63 & 321 & 56 & 312 \\
\hline
\end{tabular}

Inflow and outflow are from the quantitative pathline analysis. Aortic flow: net volume flow in the ascending aorta.

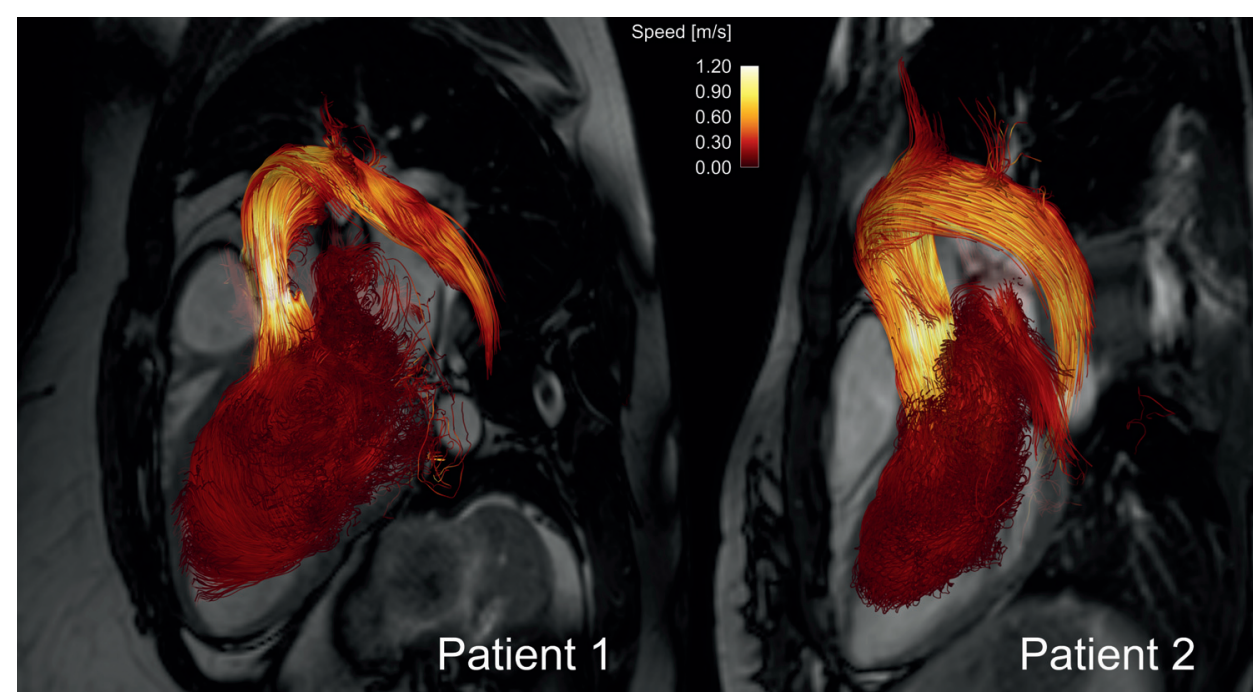

Figure 17. Pathlines covering the complete heart cycle from the spiral 4D flow measurements of the two patients. Three-chamber bSSFP images are shown for orientation. Pathlines are color-coded according to speed. Some uncorrected velocity aliasing was still present in the ascending aorta of patient 1. Patient 1: 52 years old female, ischemic heart disease dilated left ventricle. Patient 2: 19 years old female, intermittent arrhythmia. 


\subsection{Stenotic flow}

The quantification of stenotic flow is a challenging task, as many PC-MRI artifacts are more prominent in the disturbed and turbulent flow associated with a stenosis. The highly accelerating flow and the high velocities of the flow jet will cause displacement artifacts. Disturbed and turbulent velocity fluctuations will cause signal-loss and ghosting artifacts, which may lead to inaccurate flow estimation [134]. In Paper V, the feasibility of spiral kspace trajectories for the assessment of stenotic flow was evaluated. Volume flow rate, peak velocity and TKE were assessed using both spiral and Cartesian 3D PC-MRI in the same invitro phantom that was used in Paper I. Moreover, the TKE values were also compared to CFD data.

Spiral k-space sampling has been shown to be suitable for 2D PC-MRI of high speed flow jets [135] and unsteady flow systems [136]. The use of spiral trajectories enables a short TE and the time between the acquisition of the center of k-space and motion encoding is reduced compared to Cartesian sampling. The encoding of slice position is performed simultaneously with the motion encoding and is also closer to the acquisition of the center of k-space compared to Cartesian and stack-of-radials (stack-of-stars) sampling. These properties should be beneficial for 4D flow MRI of stenotic flow as displacement artifacts will be reduced. Moreover, the short TE will reduce flow-induced signal loss [81, 134].

\subsubsection{In-vitro measurements}

The feasibility of spiral k-space trajectories for the assessment of stenotic flow was evaluated by measurements of steady flow of water in an in-vitro flow phantom consisting of a straight rigid pipe with a diameter of $14.6 \mathrm{~mm}$ and a $75 \%$ area reduction stenosis.

A similar stack-of-spirals sequence as in Sections 9.2 and 9.3 was used, except that it was not time-resolved and no water-selective excitation pulse was used. The flow rate, peak velocity and TKE from the spiral PC-MRI data were compared to a conventional Cartesian acquisition. The TKE from the measurements was also compared to CFD simulations.

Flow rates 10 and $20 \mathrm{ml} / \mathrm{s}$, corresponding to Reynolds numbers 1000 and 2000, were used to obtain physiological TKE values. Two VENCs were used for these flows, one lower adapted for turbulence mapping and one higher adapted to avoid velocity aliasing. As the kinematic viscosity of water is more than 10 times less than the kinematic viscosity of blood, Reynolds numbers 1000 and 2000 resulted in very low velocities. Thus, two higher flow rates of 56 and $112 \mathrm{ml} / \mathrm{s}$ were imaged using PC-MRI velocity mapping to investigate the effect of displacement in stenotic flow. These two flow rates resulted in jet velocities of 1.4 and 2.8 $\mathrm{m} / \mathrm{s}$, respectively, representing mild and almost moderate aortic valve stenoses.

The TE of the spiral PC-MRI sequences for the mild and moderate stenotic flow cases was between 2.1 and $2.4 \mathrm{~ms}$ shorter than the TE for the corresponding Cartesian measurements. In order to correct for velocity offsets, each flow scan was accompanied by an identical scan with no flow in the phantom.

\subsubsection{Data analysis}

Peak velocity values for the mild and moderate stenotic flow were obtained by finding the maximum magnitude of the velocity in the phantom. Volume flow rates were computed at different cross-sectional planes of the phantom. In accordance with the continuity equation, the flow rate should be equal along the phantom. 
The IVSD was obtained from the measurements of Reynolds numbers 1000 and 2000 and used to compute the TKE as described in Section 4.2. Estimation of IVSD from Cartesian PCMRI has been shown to agree well with CFD simulations, laser Doppler anemometry and particle image velocimetry $[65-67,137]$. Total TKE values in the post-stenotic region of the phantom were obtained by integration of the TKE in the phantom between the center of the stenosis and six diameters downstream.

\subsubsection{Results and interpretation}

The peak velocity estimates from the different sequences and orientations show fairly good agreement (Table 11). For the mild stenotic flow, peak velocity estimates were between 1.37$1.46 \mathrm{~m} / \mathrm{s}$ for the spiral acquisition and between 1.34-1.39 m/s for the Cartesian acquisition. For the moderate stenotic flow, the peak velocities were between $2.69-2.75 \mathrm{~m} / \mathrm{s}$ for the spiral acquisition and $2.66-2.87 \mathrm{~m} / \mathrm{s}$ for the Cartesian acquisition.

As expected, there was less displacement in the spiral acquisitions compared to the Cartesian for both the mild and moderate stenotic flow. This difference was especially evident in the upslope of the flow curve when frequency encoding was performed in the principal flow direction (coronal orientation, Figure 18). While displacement was observed for both the spiral and Cartesian acquisitions with an oblique orientation (slice and frequency encoding in flow direction), the displacement was less prominent in the spiral data (Fig 19).

The estimated flow rates were quite consistent upstream of the stenosis and after 4 diameters downstream, but large variations were seen over the jet (Figure 20). Especially for the oblique orientation the flow rate seems to be overestimated by up to almost $50 \%$ in the jet. The area after the jet, where turbulent flow is expected seems to be less problematic than the jet itself.

Table 11. Peak velocity estimates.

\begin{tabular}{lll}
\hline Peak velocity $[\mathrm{m} / \mathrm{s}]$ & $\begin{array}{l}\text { Mild stenotic } \\
\text { flow }\end{array}$ & $\begin{array}{l}\text { Moderate } \\
\text { stenotic flow }\end{array}$ \\
\hline Spiral TRA & 1.37 & 2.70 \\
Cartesian TRA & 1.39 & 2.67 \\
Spiral COR & 1.39 & 2.69 \\
Cartesian COR & 1.35 & 2.66 \\
Spiral OBL & 1.46 & 2.75 \\
Cartesian OBL & 1.34 & 2.87 \\
\hline
\end{tabular}

TRA: Transverse orientation, COR: coronal, OBL: oblique. 

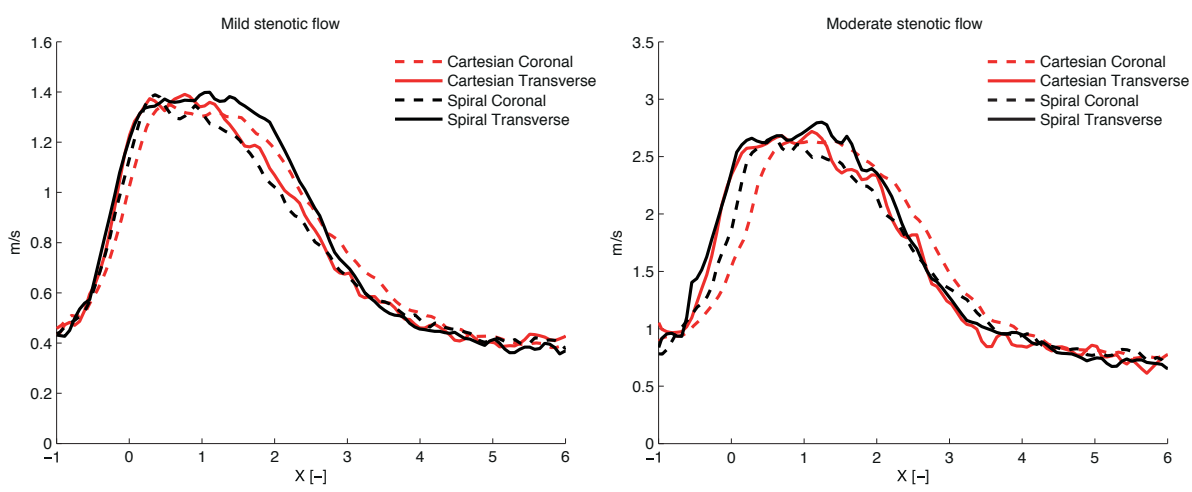

Figure 18. Plots of the velocity along the centerline of the phantom from A) the mild and B) moderate stenotic flow cases, respectively. Both Cartesian and spiral velocity data from the coronal (COR) and transverse (TRA) orientations are shown. The VENC was 150 and $300 \mathrm{~cm} / \mathrm{s}$ for the mild and moderate stenotic flow, respectively. $X$ denotes the distance from the center of the stenosis normalized by the unconstructed pipe diameter $(14.6 \mathrm{~mm})$. The principal flow direction is in the positive X-direction.
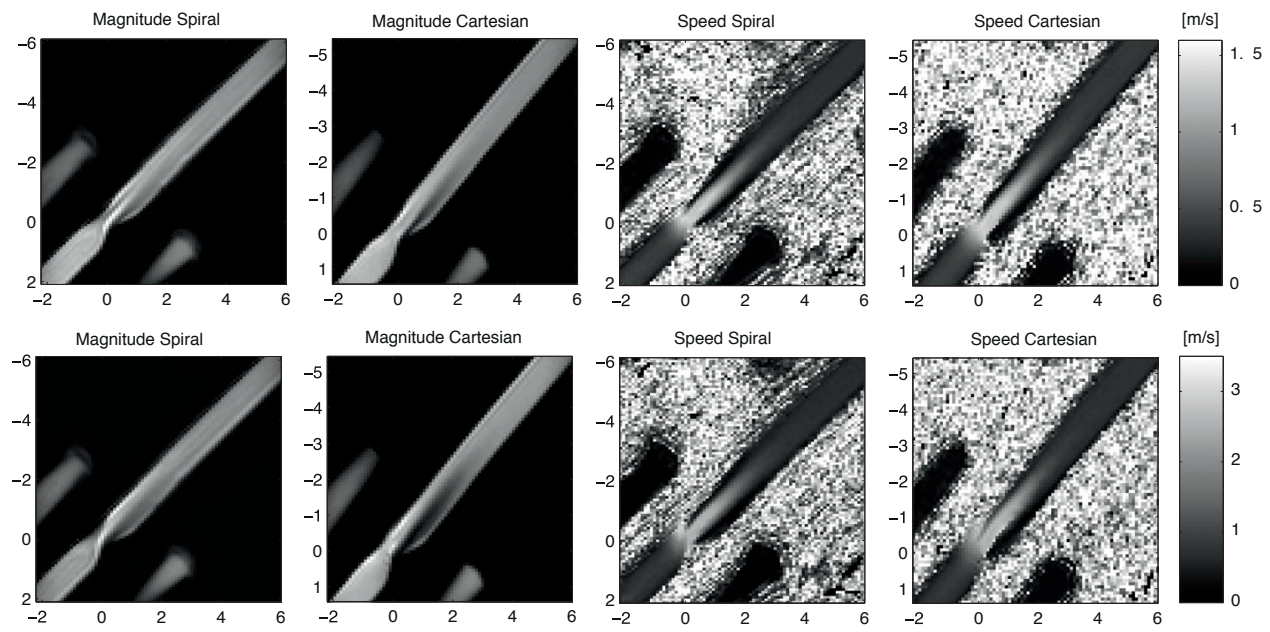

Figure 19. Magnitude and speed from spiral and Cartesian acquisitions with an oblique orientation. The upper row shows the mild stenotic flow and the bottom row the moderate stenotic flow. The VENC was 150 and $300 \mathrm{~cm} / \mathrm{s}$ for the mild and moderate stenotic flow, respectively. The stenosis is displaced towards the upper left corner of the images. Frequency encoding was used along the vertical axis and slice encoding was used along the horizontal axis. 

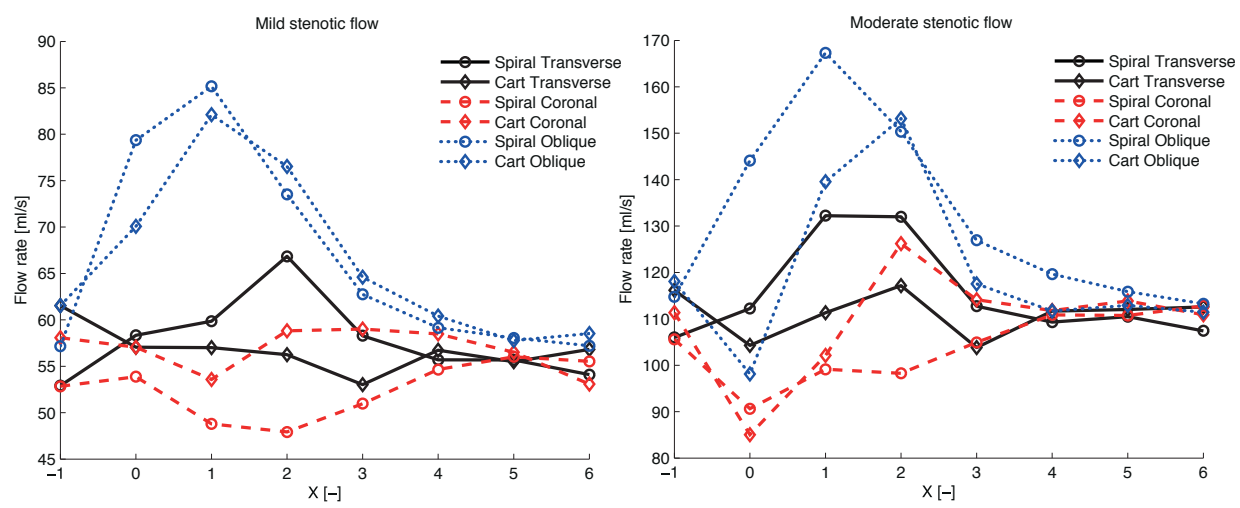

Figure 20. The volume flow rate from the mild and the moderate stenotic flow case for the different orientations: transverse, coronal and oblique. The VENC was 150 and $300 \mathrm{~cm} / \mathrm{s}$ for the mild and moderate stenotic flow, respectively. $X$ denotes the position of the cross sectional plane from which the flow rate was computed and is the distance from the center of the stenosis normalized by the unconstructed pipe diameter $(14.6 \mathrm{~mm})$. The principal flow direction is in the positive X-direction. Nominal flow rate should be $56 \mathrm{ml} / \mathrm{s}$ in the mild stenotic case and $112 \mathrm{ml} / \mathrm{s}$ in the moderate stenotic case, for all planes.

The comparison of TKE from the spiral, Cartesian and CFD data showed an overall good agreement (Figure 21). The total TKE in the post-stenotic region from the spiral and Cartesian acquisitions was similar for both Reynolds numbers 1000 and 2000 (Table 12). The total TKE from the acquisitions with coronal orientation was slightly lower compared to the transverse orientation for both the Cartesian and the spiral data. Moreover, the total TKE from the CFD data was slightly lower than the measured TKE. This difference may be caused by additional contributions from noise or shear in the IVSD estimation or by deviations between the CFD data and the measured flow.

Table 12. Total turbulent kinetic energy.

\begin{tabular}{lll}
\hline Total TKE $[\mathrm{J}]$ & Re. 1000 & Re. 2000 \\
\hline Spiral transverse & $2.08^{*} 10^{-5}$ & $9.56^{*} 10^{-5}$ \\
Cartesian transverse & $2.03^{*} 10^{-5}$ & $9.36^{*} 10^{-5}$ \\
Spiral coronal & $1.96^{*} 10^{-5}$ & $8.71^{*} 10^{-5}$ \\
Cartesian coronal & $1.94^{*} 10^{-5}$ & $7.79^{*} 10^{-5}$ \\
CFD & $1.88^{*} 10^{-5}$ & $6.82^{*} 10^{-5}$ \\
\hline
\end{tabular}

The TKE was integrated between the center of the stenosis and six diameters downstream. The VENC was 10 and $35 \mathrm{~cm} / \mathrm{s}$ for Reynolds numbers 1000 and 2000, respectively. 
a) Re.1000 Spiral Coronal

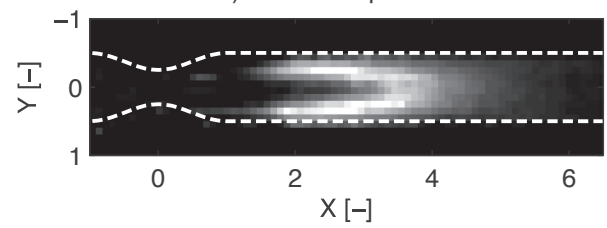

c) Re.1000 Spiral Transverse

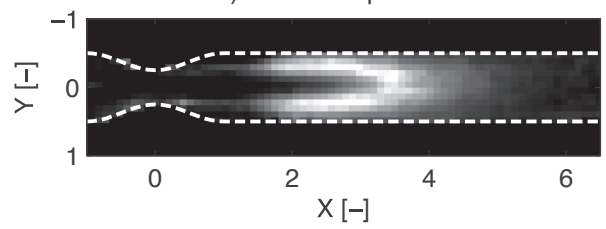

e) Re.1000 CFD

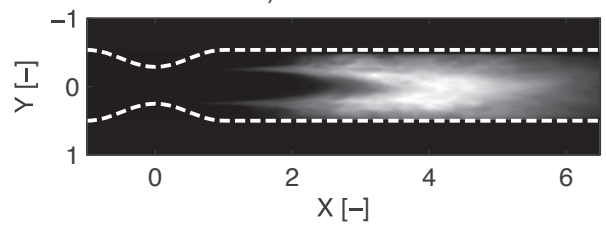

b) Re.1000 Cartesian Coronal

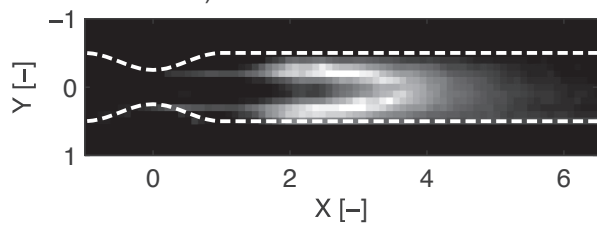

d) Re.1000 Cartesian Transverse

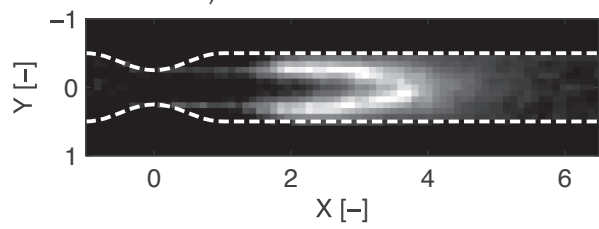

TKE $[\mathrm{Pa}]$

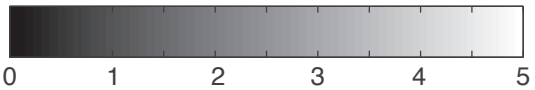

Figure 21. Images of the turbulent kinetic energy in the phantom for Reynolds number 1000, obtained using VENC $10 \mathrm{~cm} / \mathrm{s}$ from a) the spiral coronal, b) the Cartesian coronal, c) the spiral transverse, d) the Cartesian transverse 4D flow MRI measurements and e) the CFD data. $X$ and $Y$ denote the distance from the center of the stenosis normalized by the unconstructed pipe diameter (14.6 $\mathrm{mm})$. The principal flow direction is in the positive X-direction. 


\section{Discussion}

This thesis investigates the accuracy and approaches to decrease the scan time of 4D flow MRI. The accuracy evaluation focused mainly on 4D flow velocity mapping, turbulence mapping and WSS estimation. Scan times were reduced while maintaining good data quality, by using spiral k-space trajectories.

The evaluation of 4D flow MRI assessment of the complex and disturbed flows that often accompany cardiovascular diseases is often difficult, as no apparent gold standard exists for flow measurements in-vivo. Some of the artifacts that often appear in these types of flows may be reproduced in-vitro, but it is still difficult to determine the extent of the artifacts by simply studying in-vivo data only. In Paper I, PC-MRI measurements were simulated from numerical flow data describing turbulent flow. Using this approach, the flow field is known, and the accuracy and extent of different artifacts can be more easily evaluated. Hemodynamic parameters such as WSS and turbulent kinetic energy can be derived from the numerical flow data with high accuracy, and then be compared to the simulated measurements. The turbulence intensities from the simulated PC-MRI measurement were in good agreement with the turbulence from numerical flow data and in-vitro measurements. In Paper II, numerical simulations were used to evaluate the accuracy of MRI based-WSS estimation. The accuracy of different methods for WSS estimation was evaluated for a large number of parameter settings. All methods were found to be impacted by parameter settings, especially spatial resolution, and WSS values over $5 \mathrm{~N} / \mathrm{m}^{2}$ were not well resolved by any of the methods.

Many cardiovascular applications of 4D flow MRI require long scan times, sometimes around $40 \mathrm{~min}$, which prohibits extended clinical use of the technique. Although the scan time of Cartesian 4D flow MRI can be decreased using parallel imaging, one inherent problem is low scan efficiency. By using the high efficiency of spiral trajectories, scan time can be decreased without sacrificing data quality. In Papers III, IV and V a stack-of-spirals 4D flow sequence was first successfully implemented and evaluated in the thoracic aorta, left ventricle and for stenotic flow. The scan time was reduced by more than two-fold compared to a conventional Cartesian acquisition accelerated using SENSE factor 2 while maintaining good data quality.

\subsection{Simulation of PC-MRI measurements}

An Eulerian-Lagrangian approach was used for simulation of PC-MRI measurements of turbulent flow. The simulations were validated by comparison with in-vitro flow phantom measurements. Generally, good levels of agreement were found between the simulations and measurements, and artifacts such as signal drop due to intravoxel phase dispersion, ghosting, and displacement appeared both in the simulations and in-vitro measurements, demonstrating the validity of the simulations. Furthermore, the good agreement between the IVSD from the PC-MRI simulations and the CFD data further validates the accuracy of PC-MRI turbulence mapping.

Minor differences between measurements and simulations were observed, probably originating from discrepancies between the measured flow and the CFD data. A certain 
amount of difference is always expected, which is one of the reasons for using simulation of measurements instead of direct comparison between CFD and measurements, even if this direct comparison also can be very valuable.

The advantages of advanced MR-simulations are that they resemble a measurement to a high degree, the actual pulse sequences used on the scanner can be used, and it is possible to study the signal from individual spins. These advantages come at the price of a high computational burden. More simple methods such as the one used in Paper II may sometimes be more suitable, especially if the simulation of a large number of flows and parameter settings is desired.

The simulation approach used in Paper II to study the accuracy of MRI-based WSS estimates was based on the computation of the intravoxel velocity distribution. This approach can also be used to simulate the assessment of IVSD and TKE in turbulent flow, but does not include the simulation of displacement, ghosting and many other artifacts.

\subsection{MRI-based WSS estimation}

The accuracy of MRI-based WSS estimation was evaluated using a numerical simulation approach based on the computation of the intravoxel velocity distribution. Four methods based on PC-MRI and one based on FVE were studied. The effects of spatial resolution, velocity encoding, segmentation errors and partial volume artifacts were investigated for WSS between 1-20 N/m². The PC-MRI-based methods generally underestimated WSS, especially for WSS higher than $5 \mathrm{~N} / \mathrm{m}^{2}$. The FVE-based method was very sensitive to parameter settings and the amount of partial volume, and considerable errors were shown for suboptimal settings.

Spatial resolution had the most pronounced effect on the accuracy, and higher spatial resolution generally allowed estimation of higher WSS values. This is in agreement with findings from previous studies [38, 94]. However, it appears that the higher estimates were generally less precise. Thus, a higher spatial resolution may result in more accurate, but less precise, estimates. Sensitivity to parameter settings and segmentation errors, seem to increase for higher WSS estimates and the degree of underestimation also depended on the actual WSS. Cubic B-splines have been used to estimate WSS from 4D flow data without making any assumptions on the global flow profile [38]. Although this method has several advantages, such as being relatively insensitive to noise, the estimates can be expected to suffer from the same principal problems as the methods using parabolic fitting. Moreover, a recent study found that the positioning of the planes used for estimation had a larger impact on the WSS estimates than the segmentation [138].

Even in the absence of noise, all methods failed to resolve high WSS values, and the estimates were sensitive to scan settings, segmentation errors and partial volume artifacts. The results also indicate that WSS values cannot always be assumed to be linearly related to the actual WSS. In-vivo, the wall will move, and the flow will be pulsatile and far more complex, which will further deteriorate the assessment of WSS. Nevertheless, if done with care, MRI-based WSS estimation may be useful for differentiating between areas of with high and low WSS. Preferably, estimation of WSS from datasets with anisotropic voxels should be avoided, as this will result in direction-dependent estimates. The influence of spatial resolution also makes it difficult to compare estimates from measurements with different spatial resolution. As studies have indicated that it is the low WSS that is involved in the formation of 
atherosclerotic plaques and aneurysm growth [29, 33], accurate assessment of high WSS may not always be necessary.

\subsection{Spiral 4D flow}

A 4D flow pulse sequence using spiral k-space trajectories was implemented and validated for the assessment of intra-cardiac and aortic flow. Compared to conventional Cartesian sampling using SENSE factor two, a more than two-fold reduction in scan time was achieved while maintaining good data quality. No significant differences in net volume flow and cardiac output were found between the spiral TR-interleaved 4D flow sequences, the Cartesian 4D flow sequence and 2D through-plane measurements. The pathline analysis in the thoracic aorta and LV also indicate similar data quality for the spiral and Cartesian 4D flow sequences. However, the beat-interleaved sequences performed less well for diastolic flow and seem to be slightly more prone to velocity offsets, possibly due to higher sensitivity beat-to-beat variations.

In stenotic flow, the use of spiral k-space trajectories showed some benefits compared to Cartesian trajectories. While peak velocity, volume flow rate and TKE from the spiral and Cartesian acquisitions appear to agree well for all flow settings, displacement artifacts were generally less prominent in the spiral data. However, the flow estimates over the jet were less accurate for both the spiral and Cartesian data, especially for the oblique orientation, where errors of almost $50 \%$ were present. These errors are probably caused by displacement, which was most prominent with oblique orientations. The TE of the spiral sequences in the stenotic flow study was over $2 \mathrm{~ms}$ shorter than the TE of the corresponding Cartesian scans, which was not the case in the measurements of the aortic and intra-cardiac velocities, where the spiral TE was slightly longer than the Cartesian. The 1-1 spectra spatial excitation is the reason for this longer TE for the spiral acquisitions, but this should not affect the amount of displacement as the time between velocity/slice encoding and TE should remain the same for any specific VENC.

The short period of time between the bipolar gradients and the TE is advantageous in limiting displacement artifacts, but it may also result in more complex velocity offsets from eddy currents induced by the bipolar gradient. In this thesis, we noticed slightly higher offsets for the spiral sequence compared to the Cartesian, but no differences could be observed after correction. Pathline analysis will be sensitive to unsuccessfully corrected velocity offsets, especially for the low velocities in diastole.

\subsection{Future work}

In this thesis, spiral k-space trajectories were used to reduce the scan time of $4 \mathrm{D}$ flow MRI by a factor of 4 compared to un-accelerated Cartesian imaging. Other methods, such as k-t PCA or compressed sensing, have been demonstrated to achieve acceleration factors up to 8 [109$110,139]$. The combination of spiral 4D flow MRI with such methods may permit even higher acceleration factors. The combination of undersampling and non-Cartesian trajectories has been successfully applied to radial trajectories in the PC-VIPR method [93]. Similar or higher gains can be expected by using spiral trajectories, as spiral trajectories can cover a larger part of $\mathrm{k}$-space with a single readout.

For example, the GRAPPA algorithm can be used for spiral imaging without causing unreasonable reconstruction times [140]. Non-Cartesian SENSE has been used to speed up spiral 2D PC-MRI measurements of myocardial velocities [141], however, this method 
suffers from long reconstruction times [142-143]. Another appealing approach is to use variable density spirals and compressed sensing, which has shown promising results for functional MRI [144]. The efficiency of the spiral sequence can also be increased by using longer spiral readouts. However, this will lead to two problems such as higher sensitivity to off-resonance and decreased temporal resolution. The former may be solved by off-resonance correction, and the latter by using a beat-interleaved sequence. The problems found in Paper III relating to the beat-interleaved sequences must then be solved. Fully 3D spiral trajectories may further reduce the scan time relative to stack-of-spirals, but the feasibility of such trajectories for $4 \mathrm{D}$ flow remains to be investigated.

Scan time gained by undersampling will come at the cost of reduced SNR. The SNR and dynamic range of both the velocity and turbulence estimates can be increased at the cost of minor scan time penalty by using a 5-point encoding or dual VENC approach [145-146]. The quality of the velocity and turbulence estimates may be further improved using multipoint PC-MRI and Bayesian analysis [147], however, extra $k_{v}$-samples will increase the scan time.

The proposed simulation approach can be extended to include the simulation of noise, magnetic field inhomogeneity, nonlinear gradients, saturation effects and many other artifacts. Moreover, as every particle is simulated separately, it is possible to simulate spins with different magnetic properties. Computational times may be reduced by, for example, using parallel processing on graphical processing units. Simulation of PC-MRI may be used to further investigate the accuracy of, and optimize, the spiral 4D flow sequences in both laminar and turbulent flow. Only steady stenotic flow was investigated in this thesis. The performance in pulsatile stenotic flow in different geometries remains to be studied. Additionally, the feasibility of spiral 4D flow for the assessment of stenotic flow needs to be further studied by evaluating the performance in patients.

\subsection{Significance}

The possibility of combining spiral imaging with many methods to reduce scan time and improve data quality offers high flexibility. By combining parallel imaging, variable density spiral trajectories and dual VENC, the total scan time for a 4D flow measurement of the entire heart may be further reduced. The reduction of the scan time offered by the spiral k-space trajectories can save costs and time, reduce discomfort for the patient and decrease the risk of motion artifacts.

The work in this thesis further elucidates the importance of validation and assessment of the accuracy of different hemodynamic parameters. Numerical simulations provide a powerful tool for investigating the limitations of different methods in a controlled environment. Moreover, the combination of PC-MRI simulations, CFD, in-vitro and in-vivo measurements, offers the opportunity for very comprehensive study of the accuracy of PC-MRI velocity, turbulence and WSS estimation, along with many other hemodynamic parameters.

The results in Paper II show that great care must be taken in the interpretation of MRI-based WSS estimates. Differences in spatial resolutions, anisotropic voxels, and segmentation errors are some of the factors that complicate comparison of WSS estimates from different studies. Until more accurate methods presented and thoroughly validated, it may be more appropriate to see MRI-based WSS estimation as a tool for visualization, and perhaps assessment of relative differences, rather than for quantification of the actual WSS. 


\subsubsection{Clinical applications}

As 4D flow MRI can provide multidimensional flow in both ventricles and central great vessels, it is suitable for hemodynamic assessment in congenital heart disease [27, 60-62, 148]. The use of 4D flow MRI may actually decrease the combined scan and analysis time compared to using multiple 2D flow measurements to acquire the flow through, for example, shunts and valves [27, 149]. Furthermore, 4D flow MRI enables the possibility to retrospectively quantify flow through arbitrary planes in the volume, thus avoiding situations where measurements have to be repeated due to incorrect positioning of the 2D measurements. Visualization of complex flows, TKE and other hemodynamic properties can be done using pathlines and volume rendering. As an example, Figure 22 show pathlines visualizing of the flow through the right ventricle, acquired by spiral 4D flow, in a grown up congenital heart disease $(\mathrm{GUCH})$ patient with repaired tetralogy of Fallot with a dilatation in the right ventricular outflow tract.

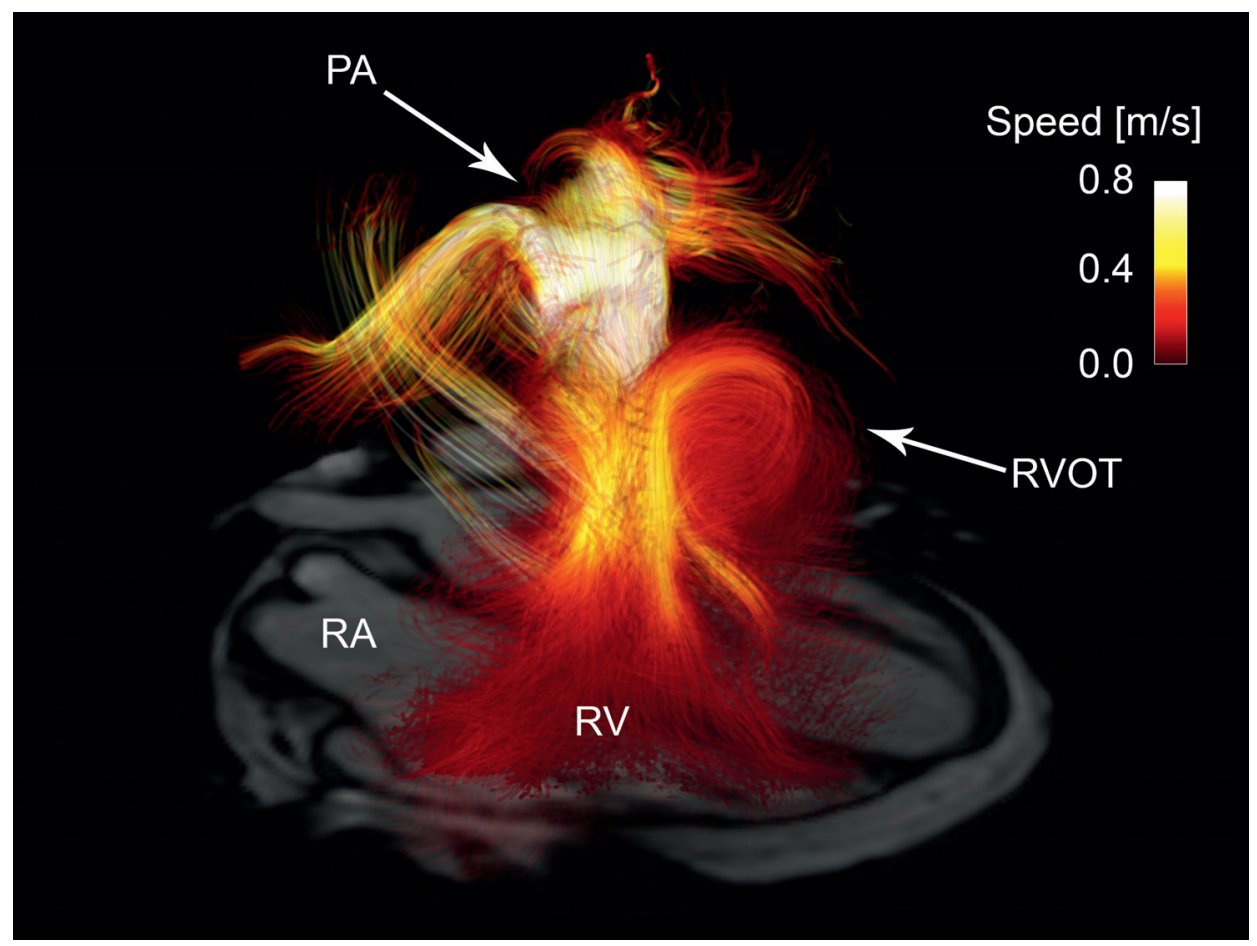

Figure 22. Pathline visualization of the flow in the right ventricle (RV) in a patient with repaired tetralogy of Fallot. RA: right atrium, RVOT: right ventricular outflow tract, PA: pulmonary artery. The flow data was acquired using spiral 4D flow MRI.

The assessment of TKE by 4D flow MRI may also be used for evaluation of obstruction defects such as aortic coarctation or valvular stenosis. The TKE can be used to evaluate the transport efficiency before and after surgical interventions; a decrease in TKE may indicate an increase in transport efficiency, and the other way around. In a comparison between the TKE from 4D flow MRI and a patient-specific CFD simulation of the flow through an aortic coarctation before and after surgery, good agreement between the integrated TKE in the post- 
stenotic region was found [66]. Moreover, it was found that, while the Reynolds number actually increased after surgery, the TKE decreased both in the measurements and CFD data.

The development of more accurate methods for the assessment of parameters such as peakvelocity, pressure drop, volume flows and TKE in stenotic flow, may offer more precise diagnosis of, for example, aortic stenosis. The pressure drop over a stenosis is often assessed from peak-velocity estimates from ultrasound by using the simplified Bernoulli equation. However, this equation is only valid for severe stenoses and will overestimate the pressure drop for insignificant and intermediate stenoses, as it assumes no pressure recovery. TKE estimates from PC-MRI have been shown to correlate with irreversible pressure loss in aortic stenosis [150]. Consequently, TKE may be a better indicator of the hemodynamic impact of moderate stenosis than the simplified Bernoulli.

The work in this thesis may facilitate expanded use of the different clinical applications of 4D flow MRI, as well as larger patient research studies. Cardiovascular blood flow assessment by fast and accurate 4D flow MRI measurements may, for example, increase the understanding of the connection between hemodynamics and cardiovascular disease, aid early identification of cardiovascular disease, and improve the timing, planning and evaluation of cardiovascular interventions. 




\section{Bibliography}

1. Ebbers, T., et al., Noninvasive measurement of time-varying three-dimensional relative pressure fields within the human heart. Journal of biomechanical engineering, 2002. 124(3): p. 288.

2. Bley, T.A., et al., Noninvasive assessment of transstenotic pressure gradients in porcine renal artery stenoses by using vastly undersampled phase-contrast $M R$ angiography. Radiology, 2011. 261(1): p. 266-273.

3. Wentland, A.L., et al., Aortic pulse wave velocity measurements with undersampled 4D flow-sensitive MRI: comparison with $2 D$ and algorithm determination. Journal of Magnetic Resonance Imaging, 2012. 37(4): p. 853-859.

4. Laffon, E., et al., Feasibility of aortic pulse pressure and pressure wave velocity MRI measurement in young adults. Journal of Magnetic Resonance Imaging, 2005. 21(1): p. 53-58.

5. Markl, M., et al., Estimation of global aortic pulse wave velocity by flow-sensitive 4D MRI. Magnetic Resonance in Medicine, 2010. 63(6): p. 1575-1582.

6. Dyverfeldt, P., et al., Quantification of intravoxel velocity standard deviation and turbulence intensity by generalizing phase-contrast MRI. Magnetic Resonance in Medicine, 2006. 56(4): p. 850-8.

7. Lopez, A.D., et al., Global and regional burden of disease and risk factors, 2001: systematic analysis of population health data. The Lancet, 2006. 367(9524): p. 17471757.

8. Kilner, P., et al., Asymmetric redirection of flow through the heart. Nature, 2000. 404: p. $759-761$.

9. Dyverfeldt, P., et al., Assessment of fluctuating velocities in disturbed cardiovascular blood flow: In vivo feasibility of generalized phase-contrast MRI. Journal of Magnetic Resonance Imaging, 2008. 28(3): p. 655-653.

10. Hope, M.D., T. Sedlic, and P. Dyverfeldt, Cardiothoracic Magnetic Resonance Flow Imaging. Journal of thoracic imaging, 2013. 28(4): p. 217-230.

11. Eriksson, J., et al., Quantification of presystolic blood flow organization and energetics in the human left ventricle. American Journal of Physiology-Heart and Circulatory Physiology, 2011. 300(6): p. H2135-H2141.

12. Carlhäll, C.J. and A. Bolger, Passing Strange Flow in the Failing Ventricle.

Circulation: Heart Failure, 2010. 3(2): p. 326-331. 
13. Mark1, M., P.J. Kilner, and T. Ebbers, Comprehensive 4D velocity mapping of the heart and great vessels by cardiovascular magnetic resonance. Journal of Cardiovascular Magnetic Resonance, 2011. 13(1): p. 1-22.

14. Ebbers, T., Flow imaging: cardiac applications of $3 D$ cine phase-contrast MRI. Current Cardiovascular Imaging Reports, 2011. 4(2): p. 127-133.

15. Fyrenius, A., et al., Three dimensional flow in the human left atrium. Heart, 2001. 86(4): p. 448-55.

16. Bolger, A.F., et al., Transit of blood flow through the human left ventricle mapped by cardiovascular magnetic resonance. J Cardiovasc Magn Reson, 2007. 9(5): p. 741-7.

17. Kilner, P.J., et al., Asymmetric redirection of flow through the heart. Nature, 2000. 404(6779): p. 759-61.

18. Kvitting, J.P.E., et al., Flow patterns in the aortic root and the aorta studied with timeresolved, 3-dimensional, phase-contrast magnetic resonance imaging: implications for aortic valve-sparing surgery. J Thorac Cardiovasc Surg, 2004. 127(6): p. 1602-7.

19. Bammer, R., et al., Time-resolved 3D quantitative flow MRI of the major intracranial vessels: Initial experience and comparative evaluation at 1.5T and 3.0T in combination with parallel imaging. Magn Reson Med, 2007. 57(1): p. 127-40.

20. Frydrychowicz, A., et al., Ascending-descending aortic bypass surgery in aortic arch coarctation: four-dimensional magnetic resonance flow analysis. J Thorac Cardiovasc Surg, 2007. 133(1): p. 260-2.

21. Hope, T.A., et al., Comparison of flow patterns in ascending aortic aneurysms and volunteers using four-dimensional magnetic resonance velocity mapping. J Magn Reson Imaging, 2007. 26(6): p. 1471-9.

22. Roes, S.D., et al., Flow assessment through four heart valves simultaneously using 3dimensional 3-directional velocity-encoded magnetic resonance imaging with retrospective valve tracking in healthy volunteers and patients with valvular regurgitation. Investigative radiology, 2009. 44(10): p. 669-675.

23. Harloff, A., et al., In vivo assessment of wall shear stress in the atherosclerotic aorta using flow sensitive 4D MRI. Magnetic Resonance in Medicine, 2010. 63(6): p. 15291536.

24. Hope, M.D., et al., Clinical evaluation of aortic coarctation with 4D flow MR imaging. Journal of Magnetic Resonance Imaging, 2010. 31(3): p. 711-718.

25. Mark1, M., et al., Time-resolved three-dimensional magnetic resonance velocity mapping of cardiovascular flow paths in volunteers and patients with Fontan circulation. European Journal of Cardio-Thoracic Surgery, 2011. 39(2): p. 206-212.

26. Eriksson, J., et al., Four-dimensional blood flow-specific markers of LV dysfunction in dilated cardiomyopathy. European Heart Journal-Cardiovascular Imaging, 2013. 14(5): p. 417-424. 
27. Valverde, I., et al., Systemic-to-pulmonary collateral flow in patients with palliated univentricular heart physiology: measurement using cardiovascular magnetic resonance $4 D$ velocity acquisition. Journal of Cardiovascular Magnetic Resonance, 2012. 14(1): p. 25.

28. Brooks, A.R., P.I. Lelkes, and G.M. Rubanyi, Gene expression profiling of human aortic endothelial cells exposed to disturbed flow and steady laminar flow. Physiological genomics, 2002. 9(1): p. 27.

29. Cheng, C., et al., Atherosclerotic lesion size and vulnerability are determined by patterns of fluid shear stress. Circulation, 2006. 113(23): p. 2744.

30. Malek, A., S. Alper, and S. Izumo, Hemodynamic shear stress and its role in atherosclerosis. Jama, 1999. 282(21): p. 2035.

31. Reneman, R.S., T. Arts, and A.P.G. Hoeks, Wall shear stress-an important determinant of endothelial cell function and structure-in the arterial system in vivo. Journal of vascular research, 2006. 43(3): p. 251-269.

32. Shaaban, A. and A. Duerinckx, Wall Shear Stress and Early Atherosclerosis A Review, in American Journal of Roentgenology. 2000, Am Roentgen Ray Soc. p. $1657-1665$

33. Boussel, L., et al., Aneurysm growth occurs at region of low wall shear stress. Stroke, 2008. 39(11): p. 2997-3002.

34. Barker, A., C. Lanning, and R. Shandas, Quantification of Hemodynamic Wall Shear Stress in Patients with Bicuspid Aortic Valve Using Phase-Contrast MRI. Annals of Biomedical Engineering, 2010. 38(3): p. 788-800.

35. Bieging, E.T., et al., In vivo three dimensional MR wall shear stress estimation in ascending aortic dilatation. Journal of Magnetic Resonance Imaging, 2011. 33(3): p. 589-597.

36. Boussel, L., et al., Phase contrast magnetic resonance imaging measurements in intracranial aneurysms in vivo of flow patterns, velocity fields, and wall shear stress. Comparison with computational fluid dynamics. Magnetic Resonance in Medicine, 2009. 61(2): p. 409-417.

37. Mark1, M., W. Wallis, and A. Harloff, Reproducibility of flow and wall shear stress analysis using flow-sensitive four-dimensional MRI. Journal of Magnetic Resonance Imaging, 2011. 33(4): p. 988-994.

38. Stalder, A., et al., Quantitative 2D and 3D phase contrast MRI: optimized analysis of blood flow and vessel wall parameters. Magnetic Resonance in Medicine, 2008. 60(5): p. 1218-1231.

39. Chang, W., et al., Hemodynamic Changes in Patients with Arteriovenous Malformations Assessed Using High-Resolution 3D Radial Phase-Contrast MR Angiography. American Journal of Neuroradiology, 2012. 33(8): p. 1565-1572. 
40. Barker, A.J., et al., Bicuspid aortic valve is associated with altered wall shear stress in the ascending aorta. Circulation: Cardiovascular Imaging, 2012. 5(4): p. 457-466.

41. Geiger, J., et al., Aortic wall shear stress in Marfan syndrome. Magnetic Resonance in Medicine, 2012. DOI: 10.1002/mrm.24562.

42. Schnell, S., et al., Three-dimensional hemodynamics in intracranial aneurysms: influence of size and morphology. Journal of Magnetic Resonance Imaging, 2013. DOI: $10.1002 / j m r i .24110$.

43. Bürk, J., et al., Evaluation of $3 D$ blood flow patterns and wall shear stress in the normal and dilated thoracic aorta using flow-sensitive 4D CMR. Journal of Cardiovascular Magnetic Resonance, 2012. 14(1): p. 84.

44. Takano, H., et al., Implication of cardiac remodeling in heart failure: mechanisms and therapeutic strategies. Internal medicine (Tokyo, Japan), 2003. 42(6): p. 465.

45. Nichols, W. and F. Michael, McDonald's Blood Flow in Arteries: Theoretical, Experimental and Clinical Principles. 2005: Hodder Arnold.

46. Sallam, A.M. and N.H. Hwang, Human red blood cell hemolysis in a turbulent shear flow: contribution of Reynolds shear stresses. Biorheology, 1984. 21(6): p. 783-97.

47. De Keulenaer, G.W., et al., Oscillatory and steady laminar shear stress differentially affect human endothelial redox state role of a superoxide-producing NADH oxidase. Circulation Research, 1998. 82(10): p. 1094-1101.

48. García-Cardeña, G., et al., Mechanosensitive Endothelial Gene Expression Profiles. Annals of the New York Academy of Sciences, 2001. 947: p. 1-6.

49. Brooks, A.R., P.I. Lelkes, and G.M. Rubanyi, Gene expression profiling of vascular endothelial cells exposed to fluid mechanical forces: relevance for focal susceptibility to atherosclerosis. Endothelium, 2004. 11(1): p. 45-57.

50. Moore, J.E., et al., Fluid wall shear stress measurements in a model of the human abdominal aorta: oscillatory behavior and relationship to atherosclerosis. Atherosclerosis, 1994. 110(2): p. 225-240.

51. Honda, H.M., et al., A complex flow pattern of low shear stress and flow reversal promotes monocyte binding to endothelial cells. Atherosclerosis, 2001. 158(2): p. 385390.

52. Caro, C., J. Fitz-Gerald, and R. Schroter, Atheroma and arterial wall shear observation, correlation and proposal of a shear dependent mass transfer mechanism for atherogenesis. Proceedings of the Royal Society of London. Series B. Biological Sciences, 1971. 177(1046): p. 109-133.

53. Chiu, J.-J., S. Usami, and S. Chien, Vascular endothelial responses to altered shear stress: pathologic implications for atherosclerosis. Annals of medicine, 2009. 41(1): p. 19-28. 
54. Shojima, M., et al., Magnitude and role of wall shear stress on cerebral aneurysm computational fluid dynamic study of 20 middle cerebral artery aneurysms. Stroke, 2004. 35(11): p. 2500-2505.

55. Ljunggren, S., A simple graphical representation of Fourier-based imaging methods. Journal of Magnetic Resonance (1969), 1983. 54(2): p. 338-343.

56. Twieg, D.B., The k-trajectory formulation of the NMR imaging process with applications in analysis and synthesis of imaging methods. Medical Physics, 1983. 10: p. 610.

57. Wigström, L., et al., Particle trace visualization of intracardiac flow using time resolved 3D phase contrast MRI. Magn Reson Med, 1999. 41(4): p. 793-9.

58. Wigström, L., L. Sjöqvist, and B. Wranne, Temporally resolved 3D phase-contrast imaging. Magn Reson Med, 1996. 36(5): p. 800-3.

59. Ebbers, T., Cardiovascular Fluid Dynamics - Methods for Flow and Pressure Field Analysis from Magnetic Resonance Imaging. 2001, Linköping University: Linköping, Sweden.

60. Bächler, P., et al., Caval blood flow distribution in patients with Fontan circulation: quantification by using particle traces from 4D flow MR imaging. Radiology, 2013. 267(1): p. 67-75.

61. Hope, T.A., et al., Evaluation of Marfan patients status post valve-sparing aortic root replacement with 4D flow. Magnetic resonance imaging, 2013. 31(9): p. 1479-1484.

62. Lorenz, R., et al., 4D flow magnetic resonance imaging in bicuspid aortic valve disease demonstrates altered distribution of aortic blood flow helicity. Magnetic Resonance in Medicine, 2013. DOI: 10.1002/mrm.24802.

63. Pelc, N., et al., Encoding strategies for three-direction phase-contrast MR imaging of flow. Journal of Magnetic Resonance Imaging, 1991. 1(4): p. 405-13.

64. Elkins, C., et al., Three-dimensional magnetic resonance velocimetry measurements of turbulence quantities in complex flow. Experiments in Fluids, 2009. 46(2): p. 285-296.

65. Dyverfeldt, P., et al., On MRI Turbulence Quantification. Magnetic Resonance Imaging, 2009. 27(7): p. 913-922.

66. Lantz, J., et al., Numerical and experimental assessment of turbulent kinetic energy in an aortic coarctation. Journal of biomechanics, 2013. 46(11): p. 1851-1858.

67. Knobloch, V., et al., Mapping mean and fluctuating velocities by Bayesian multipoint $M R$ velocity encoding-validation against $3 D$ particle tracking velocimetry. Magnetic Resonance in Medicine, 2013. DOI: 10.1002/mrm.24785.

68. Lanzer, P., et al., ECG-synchronized cardiac MR imaging: method and evaluation. Radiology, 1985. 155(3): p. 681-686. 
69. Glover, G.H. and N.J. Pelc, A rapid-gated cine MRI technique. Magnetic Resonance Annual, 1988: p. 299-333.

70. Eriksson, J., et al., Semi-automatic quantification of $4 D$ left ventricular blood flow. J Cardiovasc Magn Reson, 2010. 12(9).

71. Schrauben, E.M., et al., 3D respiratory resolved phase contrast imaging of the aorta. Journal of Cardiovascular Magnetic Resonance, 2013. 15(Suppl 1): p. P250.

72. Sigfridsson, A., et al., Five-dimensional MRI incorporating simultaneous resolution of cardiac and respiratory phases for volumetric imaging. Journal of Magnetic Resonance Imaging, 2007. 25(1): p. 113-121.

73. Dyverfeldt, P., et al., A novel MRI framework for the quantification of any moment of arbitrary velocity distributions. Magnetic Resonance in Medicine, 2011. 65(3): p. 725731.

74. Bernstein, M.A., et al., Concomitant gradient terms in phase contrast MR: Analysis and correction. Magnetic Resonance in Medicine, 1998. 39(2): p. 300-308.

75. Walker, P.G., et al., Semiautomated method for noise reduction and background phase error correction in MR phase velocity data. Journal of Magnetic Resonance Imaging, 1993. 3(3): p. 521-530.

76. Ebbers, T., et al. Higher order weighted least-squares phase offset correction for improved accuracy in phase-contrast MRI. in Proc. Intl. Soc. Mag. Reson. Med. 16 2008

77. Thunberg, P., et al., Correction for acceleration-induced displacement artifacts in phase contrast imaging. Magnetic Resonance in Medicine, 2000. 43(5): p. 734-738.

78. Thunberg, P., et al., Correction for displacement artifacts in 3D phase contrast imaging. Journal of Magnetic Resonance Imaging, 2002. 16(5): p. 591-597.

79. Xiang, Q.S., Temporal phase unwrapping for CINE velocity imaging. Journal of Magnetic Resonance Imaging, 1995. 5(5): p. 529-534.

80. Axel, L. and D. Morton, Correction of phase wrapping in magnetic resonance imaging. Medical Physics, 1989. 16: p. 284.

81. O'Brien, K., et al., Phase contrast ultrashort TE: A more reliable technique for measurement of high-velocity turbulent stenotic jets. Magnetic Resonance in Medicine, 2009. 62(3): p. 626-636.

82. Mynard, J.P., B.A. Wasserman, and D.A. Steinman, Errors in the estimation of wall shear stress by maximum Doppler velocity. Atherosclerosis, 2013. 227(2): p. 259-266.

83. Leuprecht, A., et al., Blood flow in the human ascending aorta: a combined MRI and CFD study. Journal of engineering mathematics, 2003. 47(3): p. 387-404. 
84. Renner, J., et al., A method for subject specific estimation of aortic wall shear stress. WSEAS Transactions on Biology and Biomedicine, 2009. 6(3): p. 49-57.

85. Steinman, D.A., et al., Reconstruction of carotid bifurcation hemodynamics and wall thickness using computational fluid dynamics and MRI. Magnetic Resonance in Medicine, 2002. 47(1): p. 149-159.

86. Lou, Z., W. Yang, and P. Stein, Errors in the estimation of arterial wall shear rates that result from curve fitting of velocity profiles. Journal of biomechanics, 1993. 26(45): p. 383-390.

87. Oshinski, J., et al., Determination of wall shear stress in the aorta with the use of MR phase velocity mapping. Journal of Magnetic Resonance Imaging, 1995. 5(6): p. 640647.

88. Masaryk, A., et al., In vitro and in vivo comparison of three MR measurement methods for calculating vascular shear stress in the internal carotid artery. American Journal of Neuroradiology, 1999. 20(2): p. 237.

89. Oyre, S., et al., Quantitation of circumferential subpixel vessel wall position and wall shear stress by multiple sectored three-dimensional paraboloid modeling of velocity encoded cine MR. Magnetic Resonance in Medicine, 1998. 40(5): p. 645-655.

90. Kilner, P.J., et al., Helical and retrograde secondary flow patterns in the aortic arch studied by three-directional magnetic resonance velocity mapping. Circulation, 1993. 88(5): p. 2235-2247.

91. Wetzel, S., et al., In vivo assessment and visualization of intracranial arterial hemodynamics with flow-sensitized 4D MR imaging at 3T. American Journal of Neuroradiology, 2007. 28(3): p. 433-438.

92. Cheng, C.P., D. Parker, and C.A. Taylor, Quantification of wall shear stress in large blood vessels using Lagrangian interpolation functions with cine phase-contrast magnetic resonance imaging. Annals of Biomedical Engineering, 2002. 30(8): p. 1020-1032.

93. Gu, T., et al., PC VIPR: a high-speed 3D phase-contrast method for flow quantification and high-resolution angiography. American Journal of Neuroradiology, 2005. 26(4): p. 743-749.

94. Chang, W., et al., The Effect of Spatial Resolution on Wall Shear Stress Measurements Acquired Using Radial Phase contrast Magnetic Resonance Angiography in the Middle Cerebral Arteries of Healthy Volunteers. The neuroradiology journal, 2011. 24(1): p. 115.

95. Frayne, R. and B. Rutt, Measurement of fluid-shear rate by Fourier-encoded velocity imaging. Magnetic Resonance in Medicine, 1995. 34(3): p. 378-388.

96. Carvalho, J., J. Nielsen, and K. Nayak, Feasibility of in vivo measurement of carotid wall shear rate using spiral fourier velocity encoded MRI. Magnetic Resonance in Medicine, 2010. 63(6): p. 1537-1547. 
97. Pipe, J., A simple measure of flow disorder and wall shear stress in phase contrast MRI. Magnetic Resonance in Medicine, 2003. 49(3): p. 543-550.

98. Lantz, J., R. Gårdhagen, and M. Karlsson, Quantifying turbulent wall shear stress in a subject specific human aorta using large eddy simulation. Medical Engineering \& Physics, 2012. 34(8): p. 1139-1148.

99. Lantz, J., J. Renner, and M. Karlsson, Wall shear stress in a subject specific human aorta-influence of fluid-structure interaction. International Journal of Applied Mechanics, 2011. 3(04): p. 759-778.

100. Pruessmann, K.P., et al., SENSE: sensitivity encoding for fast MRI. Magnetic Resonance in Medicine, 1999. 42(5): p. 952-962.

101. Griswold, M.A., et al., Generalized autocalibrating partially parallel acquisitions (GRAPPA). Magnetic Resonance in Medicine, 2002. 47(6): p. 1202-1210.

102. Madore, B., G.H. Glover, and N.J. Pelc, Unaliasing by Fourier-encoding the overlaps using the temporal dimension (UNFOLD), applied to cardiac imaging and fMRI. Magnetic Resonance in Medicine, 1999. 42(5): p. 813-828.

103. Tsao, J., P. Boesiger, and K.P. Pruessmann, $k$-t BLAST and $k-t$ SENSE: Dynamic MRI with high frame rate exploiting spatiotemporal correlations. Magnetic Resonance in Medicine, 2003. 50(5): p. 1031-1042.

104. Kellman, P., F.H. Epstein, and E.R. McVeigh, Adaptive sensitivity encoding incorporating temporal filtering (TSENSE) $\%$. Magnetic Resonance in Medicine, 2001. 45(5): p. 846-852.

105. Thunberg, P., M. Karlsson, and L. Wigström, Accuracy and reproducibility in phase contrast imaging using SENSE. Magnetic Resonance in Medicine, 2003. 50(5): p. 1061-1068.

106. Baltes, C., et al., Accelerating cine phase-contrast flow measurements using $k-t$ BLAST and $k$-t SENSE. Magnetic Resonance in Medicine, 2005. 54(6): p. 1430-1438.

107. Schnell, S., et al., $k$-t GRAPPA accelerated four-dimensional flow MRI in the aorta: Effect on scan time, image quality, and quantification of flow and wall shear stress. Magnetic Resonance in Medicine, 2013. DOI: 10.1002/mrm.24925.

108. Knobloch, V., P. Boesiger, and S. Kozerke, Sparsity transform k-t principal component analysis for accelerating cine three-dimensional flow measurements. Magnetic Resonance in Medicine, 2013. 70(1): p. 70:53-63.

109. Hsiao, A., et al., Evaluation of Valvular Insufficiency and Shunts with Parallelimaging Compressed-sensing 4D Phase-contrast MR Imaging with Stereoscopic 3D Velocity-fusion Volume-rendered Visualization. Radiology, 2012. 265(1): p. 87-95.

110. Hsiao, A., et al., Rapid Pediatric Cardiac Assessment of Flow and Ventricular Volume With Compressed Sensing Parallel Imaging Volumetric Cine Phase-Contrast MRI. American Journal of Roentgenology, 2012. 198(3): p. W250-W259. 
111. Bieging, E.T., et al., In vivo three-dimensional MR wall shear stress estimation in ascending aortic dilatation. Journal of Magnetic Resonance Imaging, 2011. 33(3): p. 589-597.

112. Frydrychowicz, A., et al., Images in Cardiovascular Medicine. Scimitar syndrome: added value by isotropic flow-sensitive four-dimensional magnetic resonance imaging with PC-VIPR (phase-contrast vastly undersampled isotropic projection reconstruction). Circulation, 2010. 121(23): p. e434.

113. Kecskemeti, S., et al., High resolution three-dimensional cine phase contrast MRI of small intracranial aneurysms using a stack of stars $k$-space trajectory. Journal of Magnetic Resonance Imaging, 2012. 35(3): p. 518-527.

114. Gatehouse, P., et al., Real time blood flow imaging by spiral scan phase velocity mapping. Magnetic Resonance in Medicine, 1994. 31(5): p. 504-512.

115. Pike, G.B., et al., Magnetic resonance velocity imaging using a fast spiral phase contrast sequence. Magnetic Resonance in Medicine, 1994. 32(4): p. 476-483.

116. Nayak, K.S., et al., Real-time color flow MRI. Magnetic Resonance in Medicine, 2000. 43(2): p. 251-258.

117. Börnert, P., et al., Direct comparison of 3D spiral vs. Cartesian gradient-echo coronary magnetic resonance angiography. Magnetic Resonance in Medicine, 2001. 46(4): p. 789-794.

118. Irarrázaval, P., et al., Flow properties of fast three-dimensional sequences for MR angiography. Magnetic resonance imaging, 1999. 17(10): p. 1469-1479.

119. Janiczek, R.L., et al., Three-dimensional phase contrast angiography of the mouse aortic arch using spiral MRI. Magnetic Resonance in Medicine, 2011. 66(5): p. 13821390.

120. Kadbi, M., et al. Assessment of flow and hemodynamics in the carotid artery using a reduced TE 4D flow spiral phase-contrast MRI. in Engineering in Medicine and Biology Society (EMBC), 2013 35th Annual International Conference of the IEEE. 2013: IEEE.

121. Tsai, C.M. and D.G. Nishimura, Reduced aliasing artifacts using variable-density k-space sampling trajectories. Magnetic Resonance in Medicine, 2000. 43(3): p. 452458.

122. Delattre, B.M.A., et al., Spiral demystified. Magnetic resonance imaging, 2010. 28(6): p. 862-881.

123. Doorly, D.J. and M. Ljungdahl, Computational simulation of magnetic resonance imaging techniques for velocity field measurements. Proceedings of ASME Fluids Engineering Division Summer Meeting, 1997. 
124. Lee, K.L., D.J. Doorly, and D.N. Firmin, Numerical simulations of phase contrast velocity mapping of complex flows in an anatomically realistic bypass graft geometry. Medical Physics, 2006. 33(7): p. 2621-2631.

125. Jou, L.D. and D. Saloner, A numerical study of magnetic resonance images of pulsatile flow in a two dimensional carotid bifurcation - A numerical study of MR images. Medical Engineering \& Physics, 1998. 20(9): p. 643-652.

126. Lorthois, S., et al., Numerical simulation of magnetic resonance angiographies of an anatomically realistic stenotic carotid bifurcation. Annals of Biomedical Engineering, 2005. 33(3): p. 270-283.

127. Gårdhagen, R., et al., Large Eddy Simulation of Flow Through a Stenosed Pipe. American Society of Mechanical Engineers Summer Bioengineering Conference, Marco Island, Florida, USA, 2008.

128. Gårdhagen, R., et al., Quantifying turbulent wall shear stress in a stenosed pipe using large eddy simulation. Journal of Biomechanical Engineering, 2010. 132(6): p. 061002.

129. Ahmed, S., An experimental investigation of pulsatile flow through a smooth constriction. Experimental Thermal and Fluid Science, 1998. 17(4): p. 309-318.

130. Jou, L.D., et al., Calculation of the magnetization distribution for fluid flow in curved vessels. Magnetic Resonance in Medicine, 1996. 35(4): p. 577-584.

131. Heiberg, E., et al., Design and validation of Segment-freely available software for cardiovascular image analysis. BMC medical imaging, 2010. 10(1): p. 1.

132. Kellman, P. and E.R. McVeigh, Image reconstruction in SNR units: A general method for SNR measurement $\%$. Magnetic Resonance in Medicine, 2005. 54(6): p. 1439-1447.

133. Bock, J., et al., 4D phase contrast MRI at 3 T: Effect of standard and blood-pool contrast agents on SNR, PC-MRA, and blood flow visualization. Magnetic Resonance in Medicine, 2010. 63(2): p. 330-338.

134. O'Brien, K., et al., MRI phase contrast velocity and flow errors in turbulent stenotic jets. Journal of Magnetic Resonance Imaging, 2008. 28(1): p. 210-218.

135. Nayak, K.S., B.S. Hu, and D.G. Nishimura, Rapid quantitation of high-speed flow jets. Magnetic Resonance in Medicine, 2003. 50(2): p. 366-372.

136. Tayler, A.B., et al., Time resolved velocity measurements of unsteady systems using spiral imaging. Journal of Magnetic Resonance, 2011. 211(1): p. 1-10.

137. Petersson, S., et al., Simulation of phase contrast MRI of turbulent flow. Magnetic Resonance in Medicine, 2010. 64(4): p. 1039-1046.

138. Hope, M., et al., MRI hemodynamic markers of progressive bicuspid aortic valverelated aortic disease. Journal of Magnetic Resonance Imaging, 2013. DOI: 10.1002/jmri.24362. 
139. Pedersen, H., et al., $k$-t PCA: Temporally constrained $k$-t BLAST reconstruction using principal component analysis. Magnetic Resonance in Medicine, 2009. 62(3): p. 706716.

140. Heidemann, R.M., et al., Direct parallel image reconstructions for spiral trajectories using GRAPPA. Magnetic Resonance in Medicine, 2006. 56(2): p. 317-326.

141. Simpson, R., et al., Spiral tissue phase velocity mapping in a breath-hold with non-cartesian SENSE. Magnetic Resonance in Medicine, 2013.

142. Pruessmann, K.P., et al., Advances in sensitivity encoding with arbitrary $k$-space trajectories. Magnetic Resonance in Medicine, 2001. 46(4): p. 638-651.

143. Weiger, M., et al., Sensitivity-encoded single-shot spiral imaging for reduced susceptibility artifacts in BOLD fMRI. Magnetic Resonance in Medicine, 2002. 48(5): p. 860-866.

144. Holland, D., et al., Compressed sensing reconstruction improves sensitivity of variable density spiral fMRI. Magnetic Resonance in Medicine, 2013. DOI: $10.1002 / \mathrm{mrm} .24621$

145. Johnson, K.M. and M. Markl, Improved SNR in phase contrast velocimetry with five-point balanced flow encoding. Magnetic Resonance in Medicine, 2010. 63(2): p. 349-355.

146. Nett, E.J., et al., Four-dimensional phase contrast MRI with accelerated dual velocity encoding. Journal of Magnetic Resonance Imaging, 2012. 35(6): p. 1462-1471.

147. Binter, C., et al., Bayesian multipoint velocity encoding for concurrent flow and turbulence mapping. Magnetic Resonance in Medicine, 2012. 69(5): p. 1337-1345.

148. François, C.J., et al., 4D cardiovascular magnetic resonance velocity mapping of alterations of right heart flow patterns and main pulmonary artery hemodynamics in tetralogy of Fallot. Journal of Cardiovascular Magnetic Resonance, 2012. 14(1): p. 16.

149. Brix, L., et al., Three dimensional three component whole heart cardiovascular magnetic resonance velocity mapping: comparison of flow measurements from $3 D$ and 2D acquisitions. Journal of Cardiovascular Magnetic Resonance, 2009. 11: p. 3.

150. Dyverfeldt, P., et al., Magnetic Resonance Measurement of Turbulent Kinetic Energy for the Estimation of Irreversible Pressure Loss in Aortic Stenosis. JACC: Cardiovascular Imaging, 2013. 6(1): p. 64-71. 



\section{Papers}

The articles associated with this thesis have been removed for copyright reasons. For more details about these see:

http://urn.kb.se/resolve?urn=urn:nbn:se:liu:diva-100146 